\title{
Non-uniform Hyperbolicity in Complex Dynamics
}

by

JACEK GRACZYK ${ }^{1} \&$ STANISLAV SMIRNOV 2

\footnotetext{
${ }^{1}$ Université de Paris-Sud, Mathematique, 91405 Orsay, France. (e-mail: graczyk@math.u-psud.fr)

${ }^{2}$ Université de Genève, Section de Mathématiques, 2-4 rue du Lièvre, CP 64, 1211 Genève 4, Switzerland (e-mail: Stanislav.Smirnov@math.unige.ch)

${ }^{3}$ Both authors are supported by EU Research Training Network CODY. The second author is supported by the Swiss National Science Foundation.
} 


\begin{abstract}
We say that a rational function $F$ satisfies the summability condition with exponent $\alpha$ if for every critical point $c$ which belongs to the Julia set $J$ there exists a positive integer $n_{c}$ so that $\sum_{n=1}^{\infty}\left|\left(F^{n}\right)^{\prime}\left(F^{n_{c}}(c)\right)\right|^{-\alpha}<\infty$ and $F$ has no parabolic periodic cycles. Let $\mu_{\max }$ be the maximal multiplicity of the critical points.

The objective is to study the Poincare series for a large class of rational maps and establish ergodic and regularity properties of conformal measures. If $F$ is summable with exponent $\alpha<\frac{\delta_{\text {Poin }}(J)}{\delta_{P o i n}(J)+\mu_{\max }}$ where $\delta_{P o i n}(J)$ is the Poincaré exponent of the Julia set then there exists a unique, ergodic, and non-atomic conformal measure $\nu$ with exponent $\delta_{\text {Poin }}(J)=$ $\operatorname{HDim}(J)$. If $F$ is polynomially summable with the exponent $\alpha, \sum_{n=1}^{\infty} n\left|\left(F^{n}\right)^{\prime}\left(F^{n_{c}}(c)\right)\right|^{-\alpha}<$ $\infty$ and $F$ has no parabolic periodic cycles, then $F$ has an absolutely continuous invariant measure with respect to $\nu$. This leads also to a new result about the existence of absolutely continuous invariant measures for multimodal maps of the interval.

We prove that if $F$ is summable with an exponent $\alpha<\frac{2}{2+\mu_{\max }}$ then the Minkowski dimension of $J$ is strictly less than 2 if $J \neq \mathbb{C}$ and $F$ is unstable. If $F$ is a polynomial or Blaschke product then $J$ is conformally removable. If $F$ is summable with $\alpha<\frac{1}{1+\mu_{\max }}$ then connected components of the boundary of every invariant Fatou component are locally connected. To study continuity of Hausdorff dimension of Julia sets, we introduce the concept of the uniform summability.

Finally, we derive a conformal analogue of Jakobson's (Benedicks-Carleson's) theorem and prove the external continuity of the Hausdorff dimension of Julia sets for almost all points $c$ from the Mandelbrot set with respect to the harmonic measure.
\end{abstract}

\title{
Résumé
}

Nous disons qu'une application rationnelle $F$ satisfait la condition de la sommabilité avec un exposant $\alpha$ si pour tout point critique $c$ qui appartient à l'ensemble de Julia $J$, il y a un entier positif $n_{c}$ tel que $\sum_{n=1}^{\infty}\left|\left(F^{n}\right)^{\prime}\left(F^{n_{c}}(c)\right)\right|^{-\alpha}<\infty$ et $F$ n'a pas de points périodiques paraboliques. Soit $\mu_{\max }$ la multiplicité maximale des points critiques de $F$.

L'objectif est d'étudier les séries de Poincaré pour une large classe d'applications rationnelles et d'établir les propriétés ergodiques et la regularité des mesures conformes. Si $F$ est sommable avec un exposant $\alpha<\frac{\delta_{P o i n}(J)}{\delta_{P o i n}(J)+\mu_{\max }}$, où $\delta_{P o i n}(J)$ est l'exposant de Poincaré de l'ensemble de Julia, alors il existe une unique mesure conforme $\nu$ avec l'exposant $\delta_{P o i n}(J)=$ $\operatorname{HDim}(J)$ qui est invariante, ergodique, et non-atomique. En plus, $F$ possède une mesure invariante absolument continue par rapport à $\nu$ pourvu que $\sum_{n=1}^{\infty} n\left|\left(F^{n}\right)^{\prime}\left(F^{n_{c}}(c)\right)\right|^{-\alpha}<\infty$ (la sommabilité de type polynômial) et que $F$ n'a pas de points périodiques paraboliques. Cela aboutit à un nouveau résultat sur l'existence des mesures invariantes absolument continues pour des applications multimodales d'un intervalle.

Nous démontrons que si $F$ est sommable avec un exposant $\alpha<\frac{2}{2+\mu_{\max }}$, alors la dimension de Minkowski de $J$, si $J \neq \mathbb{C}$, est strictement plus petite que 2 et $F$ est instable. Si $F$ est un polynôme ou le produit de Blaschke, alors $J$ est conformément effaçable. Si $F$ est sommable avec $\alpha<\frac{1}{1+\mu_{\max }}$, alors toute composante connexe de la frontière de chaque composante de Fatou invariante est localement connexe. Pour étudier la continuité de la dimension de Hausdorff des ensembles de Julia, nous introduisons le concept de la sommabilité uniforme.

Enfin, nous en déduisons un analogue conforme du théorème de Jakobson et BenedicksCarleson. Nous montrons la continuité externe de la dimension de Hausdorff des ensembles 
de Julia pour presque tout point de l'ensemble de Mandelbrot par rapport à la mesure harmonique. 


\section{Contents}

1 Introduction $\quad 3$

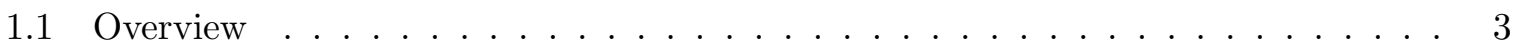

1.2 Main concepts and statements of results . . . . . . . . . . . . . 4

I Poincaré series, induced hyperbolicity, invariant measures $\quad 17$

2 Expansion along orbits $\quad 17$

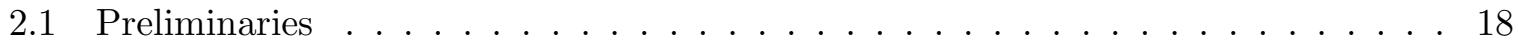

2.2 Technical sequences $\ldots \ldots \ldots \ldots \ldots \ldots$

2.3 Constants and scales . . . . . . . . . . . . . . . . . . . . . 19

2.4 Types of orbits . . . . . . . . . . . . . . . . . . 20

3 Specification of orbits $\quad \mathbf{2 5}$

3.1 Inductive decomposition of backward orbits . . . . . . . . . . . . 26

3.2 Estimates of expansion . . . . . . . . . . . . . . . . . . 28

3.3 Summability along backward orbits . . . . . . . . . . . . . . . . 32

4 Poincaré series $\quad 33$

5 Induced hyperbolicity and conformal measures $\quad 36$

5.1 Inductive procedure with a stopping rule . . . . . . . . . . . . 36

5.2 Most points go to large scale infinitely often . . . . . . . . . . . . . . 37

5.3 Conformal measures . . . . . . . . . . . . . . . . . . . 39

5.4 Frequency of passages to the large scale . . . . . . . . . . . . . . 41

6 Invariant measures $\quad 43$

6.1 Polynomial summability condition . . . . . . . . . . . . . . . 43

6.2 Ergodic properties . . . . . . . . . . . . . . . . 45

II Geometry, rigidity, perturbations $\quad 48$

$\begin{array}{lll}7 & \text { Fractal structure } & \mathbf{4 8}\end{array}$

7.1 Average contraction of preimages . . . . . . . . . . . . . . 48

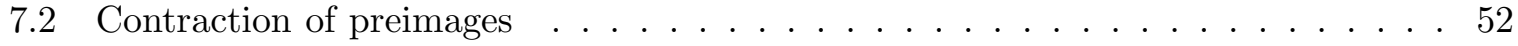

7.3 Most points go to large scale infinitely often . . . . . . . . . . . . 53

8 Dimensions and conformal measures $\quad \mathbf{5 5}$

8.1 Fractal dimensions . . . . . . . . . . . . . . . . . . . . . 55

8.2 Multifractal analysis . . . . . . . . . . . . . . . . 56 
9 Removability and rigidity $\quad \mathbf{5 9}$

9.1 Conformal removability and strong rigidity . . . . . . . . . . . . 59

9.2 Dynamical removability and rigidity . . . . . . . . . . . . . . 59

10 Integrability condition and invariant measures. $\quad 61$

10.1 Ruelle-Perron-Frobenius transfer operator. . . . . . . . . . . . . . . . 62

10.2 Regularity of invariant densities . . . . . . . . . . . . . . . . 64

10.3 Analytic maps of interval . . . . . . . . . . . . . . . 66

$\begin{array}{ll}11 \text { Geometry of Fatou components } & \mathbf{6 8}\end{array}$

11.1 Integrable domains . . . . . . . . . . . . . . . . . . 68

11.2 Local connectivity and continua of convergence . . . . . . . . . . . . . 70

11.3 Wandering continua . . . . . . . . . . . . . . . 71

12 Uniform summability condition $\quad 71$

13 Unicritical polynomials $\quad \mathbf{7 6}$

13.1 Renormalizations . . . . . . . . . . . . . . . . . . . . . . . . . . . . . .

13.2 Radial limits . . . . . . . . . . . . . . . . . . . . . 77 


\section{Introduction}

\section{$1.1 \quad$ Overview}

The Poincaré series is a basic tool in the theory of Kleinian groups. It is used to construct and study conformal densities and dimensions of the limit set. Patterson and Sullivan proved that the critical exponent (the Poincaré exponent) is equal to the Hausdorff dimension of the limit set for Fuchsian and non-elementary geometrically finite Kleinian groups.

We focus on estimates of the Poincaré series in rational dynamics. From this perspective, we address the problem of regularity of conformal measures. We propose to study rational maps satisfying the summability condition, which requires, roughly speaking, only a polynomial growth of the derivative along critical orbits. Rational maps with parabolic periodic points are non-generic and for simplicity we exclude them from our considerations.

In the class of rational maps satisfying the summability condition, we prove the counterpart of Sullivan's result that conformal measures with minimal exponent are ergodic (hence unique) and non-atomic. To pursue properties of the Poincaré series for rational maps we introduce a notion of a restricted Poincaré series which is also well-defined for points in the Julia set. This notion leads to new estimates, particularly implying that the convergence property of the Poincaré series is "self-improving." This turns out to be an underlying reasons for regularity properties of conformal measures on Julia sets. Also, the divergence of the Poincaré series with the Poincaré exponent (infimum of exponents with converging Poincaré series) is an immediate consequence. A different definition of the Poincaré exponent and its relation with various dynamical dimensions can be found in [38].

One of the central problems in the theory of iteration of rational functions is to estimate the Hausdorff dimension of Julia sets which are not the whole sphere and investigate their fractal properties. It is believed that rational functions with metrically small Julia sets should possess certain weak expansion property. We prove that the Poincaré exponent coincides with the Hausdorff dimension of the Julia set $J$ and $\operatorname{HDim}(J)<2$ unless $J=\hat{\mathbb{C}}$ for rational functions satisfying the summability condition with an exponent $\alpha<\frac{2}{\mu_{\max }+2}$. These results bear some relationship with a recent result of C. Bishop and P. Jones [6] which says that for finitely generated Kleinian groups if the limit set has zero area then the Poincaré exponent is equal to the Hausdorff dimension of the limit set.

Perhaps, the most famous problem in the iteration theory of rational functions is whether a given system can be perturbed to a hyperbolic one or not. It is widely believed that this should be possible (the Fatou conjecture), at least in the class of polynomials. It is well known, [29], that if the Julia set of a polynomial is of Lebesgue measure zero then the polynomial can be perturbed to a hyperbolic one. In general, despite much effort, a very limited progress was achieved towards proving the Fatou conjecture. The real Fatou conjecture was proved in [26] . We use the recent result of [24] to prove strong instability of polynomials satisfying the summability condition. This both strengthens and generalizes the results of [39] in the class of polynomials and Blaschke products.

The summability condition was proposed in one-dimensional real unimodal dynamics [34] as a weak condition which would guarantee the existence of absolutely continuous measures 
with respect to the one-dimensional Lebesgue measure. M. Aspenberg proved in [1] that the class of rational functions which satisfy the Collet-Eckmann condition is of positive Lebesgue measure in the space of all rational functions of a given degree (see also a closely related paper of M. Rees [40])

From the point of view of measurable dynamics and ergodic theory, the existence of regular invariant measures is of crucial importance. A dynamical analogue of the one-dimensional Lebesgue measure on Julia set is given by the "geometric measures" (conformal measures with minimal exponents). We study regularity and ergodic properties of conformal measures and determine whether the dynamics admits the existence of absolutely continuous invariant measures with respect to a given conformal measure. The problem is twofold and involves both dynamical and measure theoretical estimates.

Another problem we look at is local connectivity of Julia sets and the existence of wandering continua. In order to pursue the continuity of the Hausdorff dimension of Julia sets we introduce a uniform convergene for rational maps satisfying the summability condition. Finally, we discuss applications of our theory to the study of complex unicritical polynomials $z^{d}+c$. In this setting, we formulate a complex analogue of Jacobson and Benedicks-Carleson's theorems.

Non-uniform hyperbolicity. The concept of non-uniform hyperbolicity is slightly vague and depends on varying backgrounds and motivations. It is difficult to find a single formulation of this property. Our approach emphasizes measure theoretical aspects of the system, which should be hyperbolic on the average. Loosely speaking, given a non-hyperbolic system, one tries to make it hyperbolic by taking only pieces of the phase space and a high iterate of the map on each piece. If it is possible to find such pieces almost everywhere, we say that the system induces hyperbolicity or is non-uniformly hyperbolic with respect to a given measure. Of course, we are interested only in natural measures such as the Lebesgue measure when $J=\hat{\mathbb{C}}$ and geometric measures (see Definition 1.3 and the following discussion) when $J \neq \mathbb{C}$. This approach originates from the work of Jakobson [23] on the abundance of absolutely continuous invariant measures and was also followed in a similar way by Benedicks and Carleson [3, 4]. The concept of induced hyperbolicity plays also a central role in the proof of the real Fatou conjecture, see [19].

For rational functions $F$ satisfying the summability condition we prove induced hyperbolicity with respect to unique geometric measure on $J$ (Theorem 3 ). The induced hyperbolicity yields that the Julia set is of Lebesgue measure zero whenever $J \neq \hat{\mathbb{C}}$, see also [7]. In many cases (e.g. under polynomial summability condition) we prove a stronger version of non-uniform hyperbolicity, namely the existence of a unique absolutely continuous invariant measure $\sigma$ with respect to the geometric measure. The measure $\sigma$ is ergodic, mixing, and has positive Lyapunov exponent.

\subsection{Main concepts and statements of results}

Summability conditions and maximal multiplicity. Before stating our main theorems, we make a few technical remarks. For simplicity we assume that no critical point belongs to 
another critical orbit. Otherwise all theorems remain valid with the following amendment: a "block" of critical points

$$
F: \quad c_{1} \mapsto \ldots \mapsto c_{2} \mapsto \ldots \ldots \mapsto c_{k}
$$

of multiplicities $\mu_{1}, \mu_{2}, \ldots, \mu_{k}$ enters the statements as if it is a single critical point of multiplicity $\prod \mu_{j}$.

If the Julia set is not the whole sphere, we use the usual Euclidean metric on the plane, changing the coordinates by a Möbius transformation so that $\infty$ belongs to a periodic Fatou component, and doing all the reasoning on a large compact containing the Julia set. Alternatively (and also when $J=\hat{\mathbb{C}}$ ) one can use the spherical metric.

Define $\sigma_{n}:=\min _{c \in \operatorname{Crit}}\left\{\left|\left(F^{n}\right)^{\prime}(F c)\right|\right\}$, where minimum is taken over all critical points in the Julia set whose forward orbits do not contain other critical points. Many properties will take into account $\mu_{\max }$ - the maximal multiplicity of critical points in the Julia set (calculated as above, if there are any critical orbit relations).

Definition 1.1 Suppose that $F$ is a rational function without parabolic periodic points. We say that $F$ satisfies the summability condition with exponent $\alpha$ if

$$
\sum_{j=1}^{\infty}\left(\sigma_{j}\right)^{-\alpha}<\infty
$$

If a stronger inequality

$$
\sum_{j=1}^{\infty} j \cdot\left(\sigma_{j}\right)^{-\alpha}<\infty
$$

holds, then we say that $F$ satisfies the polynomial summability condition with exponent $\alpha$.

We recall that $F$ satisfies the Collet-Eckmann condition if there exist $C>0$ and $\Lambda>1$ such that $\sigma_{n} \geq C \Lambda^{n}$ for every positive $n$. Contrary to the Collet-Eckmann case, the summability condition allows strong recurrence of the critical points. Generally, it is not true that the critical value of a summable rational map has infinitely many univalent passages to the large scale (counterexample given e.g. by a quadratic Fibonacci polynomial), compare Theorem 3.

Poincaré series. We call a point $z$ admissible if it does not belong to $\bigcup_{i=0}^{\infty} F^{n}$ (Crit). Take an admissible point $z$ and assume that $F$ has no elliptic Fatou components and $J \neq \hat{\mathbb{C}}$. We define the Poincaré series by

$$
\boldsymbol{\Sigma}_{\delta}(z):=\sum_{n=1}^{\infty} \sum_{y \in F^{-n} z}\left|\left(F^{n}\right)^{\prime}(y)\right|^{-\delta} .
$$

The series converges for every $\delta>\delta_{\text {Poin }}(z)$ and the minimal such $\delta_{\text {Poin }}(z)$ is called the Poincaré exponent (of $F$ at the point $z$ ). By standard distortion considerations, if $\mathcal{F}$ is a component of the Fatou set, then for all admissible $z \in \mathcal{F}$ Poincaré exponents coincide, so we set

$$
\delta_{\text {Poin }}(\mathcal{F}):=\delta_{\text {Poin }}(z) .
$$


We define the Poincaré exponent by

$$
\delta_{\text {Poin }}(J):=\max \left\{\delta_{\text {Poin }}(\mathcal{F})\right\},
$$

(As Theorem 1 shows, we can alternatively set $\delta_{\text {Poin }}(J):=\inf \left\{\delta_{P \text { oin }}(x): x \in \hat{\mathbb{C}}\right\}$ ). A wellknown area estimate shows that $\delta_{P o i n}(J) \leq 2$. A natural question arises if the Poincaré exponents $\delta_{\text {Poin }}(\mathcal{F})$ in different Fatou components coincide and if $\delta_{P o i n}(J)<2$. By the analogy with the theory of Kleinian groups, we say that $F$ is of divergent (convergent) type if the Poincaré series $\boldsymbol{\Sigma}_{\delta_{\text {Poin }}}(z)$ diverges (converges) for every component $\mathcal{F}$ of the Fatou set and every admissible $z \in \mathcal{F}$. Clearly, hyperbolic rational maps satisfy the summability condition with any positive exponent $\alpha$. By Theorem 1, they are all of divergent type. In general, rational maps of the divergent type can be viewed as weakly hyperbolic systems. It would be interesting to study this property from an abstract point of view.

To address the above problems, we consider the Poincaré series as a function of $z \in \hat{\mathbb{C}} \backslash J$ and study its limiting behavior when $z$ approaches the Julia set. We use the concept of a restricted Poincaré series to study the dynamics of inverse branches of $F$ independently from the fact whether their domains intersect $J$ or not.

Let $\mathcal{H}(z, \Delta)$ be the set of all preimages of $z$ such that the ball $B(z, \Delta)$ can be pulled back univalently along the corresponding branch.

Definition 1.2 The restricted Poincaré series for a number $\Delta>0$ and $z \in \hat{\mathbb{C}}$ is defined by

$$
\Sigma_{\delta}^{\Delta}(z):=\sum_{n=1}^{\infty} \sum_{y \in F^{-n}(z) \cap \mathcal{H}(z, \Delta)}\left|\left(F^{n}\right)^{\prime}(y)\right|^{-\delta} .
$$

The definitions of the Poincaré exponents assume that the complement of $J$ is non-empty. Should $J$ coincide with the whole sphere, we set $\delta_{\text {Poin }}(J):=2$. We will prove that under the summability condition the convergence of the Poincaré series is an open property. This means that $F$ is of divergent type. The proof will use the restricted Poincaré series and a generalized "area estimate." Intriguingly, our technique allows us to compare different $\delta_{\text {Poin }}(\mathcal{F})$ through perturbations of the Poincaré series near the critical points $c \in J$ of the maximal multiplicity. These points appear to be in the stability locus of $\delta_{P o i n}(z)$.

Theorem 1 Suppose that $F$ satisfies the summability condition with an exponent

$$
\alpha<\frac{\delta_{\text {Poin }}(J)}{\mu_{\max }+\delta_{\text {Poin }}(J)} .
$$

We have that

- the Poincaré series with the critical exponent $\delta_{\text {Poin }}(J)$ diverges for every point $z \in \hat{\mathbb{C}}$,

- if $z$ is at a positive distance to the critical orbits and $c$ is a critical point of the maximal multiplicity then

$$
\delta_{\text {Poin }}(z)=\delta_{\text {Poin }}(c)=\delta_{\text {Poin }}(J)=\inf \left\{\delta_{\text {Poin }}(x): x \in \hat{\mathbb{C}}\right\}
$$


If $J=\hat{\mathbb{C}}$ then by our convention, $\delta_{\text {Poin }}(J)=2$. Hence, the equality

$$
\delta_{\text {Poin }}(J)=\inf \left\{\delta_{\text {Poin }}(x): x \in \hat{\mathbb{C}}\right\}
$$

of Theorem 1 can be regarded as an alternative definition of the Poincaré exponent when $J=\hat{\mathbb{C}}$.

Conformal and geometric measures. Conformal or Patterson-Sullivan measures are dynamical analogues of Hausdorff measures and capture important (hyperbolic) features of the underlying dynamics.

Definition 1.3 Let $F$ be a rational map with the Julia set $J$. A Borel measure $\nu$ supported on $J$ is called conformal with an exponent $p$ (or p-conformal) if for every Borel set $A$ on which $F$ is injective one has

$$
\nu(F(B))=\int_{B}\left|F^{\prime}(z)\right|^{p} d \nu .
$$

As observed in [45], the set of pairs $(p, \nu)$ with $p$-conformal measure $\nu$ is compact (in the weak-* topology). Hence, there exists a conformal measure with the minimal exponent

$$
\delta_{\text {conf }}:=\inf \{p: \exists \text { a } p \text {-conformal measure on } J\} .
$$

The minimal exponent $\delta_{\text {conf }}$ is also called a conformal dimension of $J$.

However, conformal measures might have extremely bad analytical properties, in particular they can be atomic. In this context it is rather surprising that the most important conformal measures, namely these with minimal exponents, have many good analytical properties in the class of rational maps which satisfy the summability condition.

A hyperbolic Julia set has the Hausdorff dimension strictly less than 2 and a finite positive Hausdorff measure in its dimension, [45]. In the hyperbolic case, there is a unique conformal measure which coincides with the normalized Hausdorff measure. For non-hyperbolic maps the situation is much more complicated. A construction of conformal measures for Kleinian groups was proposed by Patterson. The same construction was implemented by Sullivan for rational functions [45]. In Patterson-Sullivan approach conformal measures are constructed through the dynamics in the Fatou set. This external construction favors conformal measures with "inflated" exponents and can be briefly summarized as follows: Assume that $J \neq \hat{\mathbb{C}}$ and $F$ has no neutral cycles. Let $z \in \hat{\mathbb{C}} \backslash J$. If $\boldsymbol{\Sigma}_{\delta_{\text {Poin }}}(z)$ diverges then, for any $p>\delta_{\text {Poin }}(z)$ we consider $v_{p}$ defined by

$$
\nu_{p}:=\frac{1}{\Sigma_{p}} \sum_{n=1}^{\infty} \sum_{y \in F^{-n}(z)} \frac{1_{y}}{\left|\left(F^{n}\right)^{\prime}(y)\right|^{p}},
$$

where $1_{y}$ is a Dirac measure at $y$. A weak limit of such atomic measures when $p \rightarrow \delta_{\text {Poin }}(z)$ defines a $\delta_{\text {Poin }}(z)$-conformal measure on the Julia set. If $\boldsymbol{\Sigma}_{\delta_{\text {Poin }}}(z)$ converges then the construction can be repeated in the same way after multiplying each term of $\boldsymbol{\Sigma}_{p}$ by a factor $h\left(\left|\left(F^{n}\right)^{\prime}(y)\right|^{-p}\right)$, where $h$ is the Patterson function. The function $h$ tends to 1 subexponentialy but still makes the series $\boldsymbol{\Sigma}_{p}$ divergent for $p=\delta_{\text {Poin }}(z)$. Clearly,

$$
\delta_{\text {conf }} \leq \delta_{\text {Poin }} .
$$


An alternative "internal" construction was carried out by Denker and Urbanski, [12, 13]. It uses increasing forward invariant subcompacts inside the Julia set, free of critical points, to distribute probabilistic Borel measures $\nu_{n}$ with Jacobians bounded respectively by $\left|F^{\prime}(z)\right|^{p_{n}}$. In the limit, these approximating measures become conformal with exponent equal to $\sup p_{n}=$ $\delta_{\text {conf }},[12,13]$. Recall that the hyperbolic dimension $\operatorname{HypDim}(J)$ of the Julia set $J$ is equal to the supremum of the Hausdorff dimensions of all hyperbolic subsets of $J$. An important geometric consequence of the Denker-Urbanski construction is

$$
\delta_{\text {conf }}=\operatorname{HypDim}(J) \text {. }
$$

If additionally $\delta_{\text {conf }}=\operatorname{HDim}(J)$ then every $\delta_{\text {conf }}$-conformal measure $\nu$ is called a geometric measure. It is an important open question, whether $\delta_{\text {conf }}=\operatorname{HDim}(J)$ always holds, and in general very little is known about the existence and regularity properties of geometric measures (cf. [41, 37, 47]). It is not even known if a geometric measure has to be unique and non-atomic. A possible pathology is due to the presence of critical points in $J$ of convergent type. Indeed, if $\boldsymbol{\Sigma}_{p}(c)$ converges for $p \leq 2$ and $c \in$ Crit then $\nu_{p}$ defined by (1) with $y=c$ is a $p$-conformal atomic measure.

We prove that if a rational function satisfies the summability condition with an exponent $\alpha<\frac{2}{2+\mu_{\max }}$, then, for every $p>\delta_{\text {conf }}$, there exists an atomic $p$-conformal measure with an atom at a critical point. In contrast to that, the geometric measures are non-atomic in this class. The majority of work in the area is based on Denker-Urbanski construction. We come back to the origins and focus on the Patterson-Sullivan approach.

Every conformal measure of $F$ has an exponent $p \in\left[\delta_{\text {conf }}, \infty\right)$. The set of all such $p$ forms a conformal spectrum of $F$. We distinguish an atomic part of the spectrum consisting of all $p \in\left[\delta_{\text {conf }}, \infty\right)$ for which there exist only atomic conformal measures, a continuous part corresponding to exponents for which there exist only non-atomic conformal measures, and a mixed part (possibly empty) gathering all $p$ for which there exist both atomic and non-atomic $p$-conformal measures.

Definition 1.4 We say that the conformal spectrum of $F$ is hyperbolic if its mixed part is empty and continuous part is equal to $\left\{\delta_{\text {conf }}\right\}$.

Theorem 2 Suppose that a rational function $F$ satisfies the summability condition with an exponent

$$
\alpha<\frac{\delta_{\text {Poin }}(J)}{\mu_{\max }+\delta_{\text {Poin }}(J)} .
$$

Then

- there is a unique non-atomic conformal measure $\mu$,

- measure $\mu$ is ergodic and its exponent is equal to $\delta_{\text {conf }}=\delta_{\text {Poin }}(J)$,

- if $F$ has critical points in the Julia set then for every $\delta>\delta_{\text {conf }}$ there exists an atomic $\delta$-conformal measure supported on the backward orbits of the critical points, 
- every conformal measure has no atoms outside of the set of the critical points of $F$.

In particular, every rational function which satisfies the summability condition with an exponent $\alpha<\frac{\delta_{\text {Poin }}(J)}{\mu_{\max }+\delta_{\text {Poin }}(J)}$ has a hyperbolic conformal spectrum. Since every hyperbolic rational map has no critical points in the Julia set, its conformal spectrum is trivial and consists of only one point $\left\{\delta_{\text {conf }}\right\}$.

Observe that if we know that every geometric measure is ergodic, then in fact it must be unique. Indeed, if there were two such measures $\nu_{1}$ and $\nu_{2}$, then $\nu_{3}:=\frac{\nu_{1}+\nu_{2}}{2}$ is obviously a non-ergodic geometric measure, a contradiction.

The problem of ergodicity of conformal measures was raised before. In [37] it is proved that a number of ergodic components of conformal measures $\nu$ for the so-called Collet-Eckmann rational maps is finite and not exceeding the number of critical points. This is not enough to conclude uniqueness of a geometric measure. The assertion of Theorem 2 is valid only for conformal measures with minimal exponent. In general, if $p>\delta_{\text {conf }}$ and $F$ has more than one critical point of the maximal multiplicity, then there exist non-ergodic $p$-conformal measures. Indeed, by Theorem 1 , if $\mu(c)=\mu_{\text {max }}$ then $\delta_{\text {Poin }}(c)=\delta_{\text {conf } f}$. Measures $\nu(c)$ defined by (1) are $p$-conformal and their convex sum has exactly $\#\left\{c: \mu(c)=\mu_{\max }\right\}$ ergodic components.

Induced hyperbolicity. Consider the set $J_{*, \epsilon}$ of all points $x \in J$ which $\epsilon$-frequently go to the large scale of radius $r$, namely:

$$
\exists n_{j} \rightarrow \infty: F^{n_{j}} \text { is univalent on } F^{-n_{j}}\left(B\left(F^{n_{j}} x, r\right)\right), \quad\left|\left(F^{n_{j+1}}\right)^{\prime}(x)\right|<\left|\left(F^{n_{j}}\right)^{\prime}(x)\right|^{1+\epsilon},
$$

where $F^{-n_{j}}\left(B\left(F^{n_{j}} x, r\right)\right)$ stands for its connected component, containing $x$.

Theorem 3 (Strong induced hyperbolicity) Suppose that a rational function $F$ satisfies the summability condition with an exponent

$$
\alpha<\frac{\delta_{\text {Poin }}(J)}{\delta_{\text {Poin }}(J)+\mu_{\max }} .
$$

Then there exists $r>0$ so that for every $\epsilon>0$ almost every point with respect to a unique

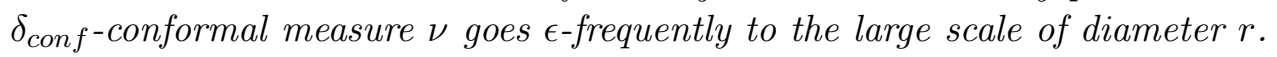

Invariant measures. The summability condition was introduced in real dynamics in [34] to study absolutely continuous invariant measures (shortly acim) with respect to the Lebesgue measure. In the conformal setting when conservative dynamics is often concentrated on fractal sets with zero area, a concept of an acim encounters some hurdles. In this situation, it is natural to study absolutely continuous invariant measures with respect to conformal measures. This approach was already adopted by Przytycki, who proved that a rational Collet-Eckmann map has an acim with respect to a $p$-conformal measure $\nu$, provided that $\nu$ is regular enough along critical orbits [36]. This regularity was verified only in particular cases (cf. Tsujii's condition 
in [36]) and can be expressed by an integral condition (in [37] a slightly weaker condition was considered): there exists $C>0$ so that for all $i>0$

$$
\sup _{c \in \text { Crit }} \int\left|z-F^{i}(c)\right|^{-p\left(1-\frac{1}{\mu_{\max }}\right)} d \nu<C
$$

The scope of validity of this condition is not known even in the Collet-Eckmann setting. We will call this condition the integrability condition with an exponent $\eta:=p\left(1-\frac{1}{\mu_{\max }}\right)$.

Surprisingly, we do not need to assume this condition to obtain an absolutely continuous invariant measure with respect to a non-atomic conformal measure, if $F$ satisfies the polynomial summability condition.

Theorem 4 Suppose that a rational function $F$ satisfies the polynomial summability condition with an exponent

$$
\alpha<\frac{\delta_{\text {Poin }}(J)}{\delta_{\text {Poin }}(J)+\mu_{\max }} .
$$

Then $F$ has a unique absolutely continuous invariant measure $\sigma$ with respect to a unique $\delta_{\text {Poin }}(J)$-conformal measure $\nu$. Moreover, $\sigma$ is ergodic, mixing, exact, has positive entropy and Lyapunov exponent.

If the integrability condition is satisfied, then we have the following theorem.

Theorem 5 If $F$ satisfies the summability condition with an exponent

$$
\alpha<\frac{\delta_{\text {Poin }}(J)}{\delta_{\text {Poin }}(J)+\mu_{\max }},
$$

and a unique $\delta_{\text {Poin }}(J)$-conformal measure $\nu$ is $\delta_{\text {Poin }}(J)\left(1-\frac{1}{\mu_{\max }}\right)$-integrable then $F$ has a unique and ergodic absolutely continuous invariant measure.

Observe that 2-dimensional Lebesgue measure becomes a geometric (conformal) measure, when $J=\hat{\mathbb{C}}$. We conclude, that in this case $F$ has an absolutely continuous invariant measure with respect to 2-dimensional Lebesgue measure if it satisfies the summability condition with an exponent $\alpha<\frac{2}{2+\mu_{\max }}$. We also study ergodic and regularity properties of the absolutely continuous invariant measures.

Multimodal maps. Another important application of our techniques lies in the dynamics of analytic multimodal maps of a compact interval with non-empty interior. Contrary to the unimodal case (maps with exactly one local extremum), there are few results about the existence of absolutely continuous invariant measures, [8]. The only available results are either of perturbative nature (analogues of Jakobson's result in the quadratic family, i.e. they require some transversality assumptions in one-parameter families) or impose some semi-unimodal conditions. One of the most general results [46] states that for a generic $C^{2}$ families of multimodal maps there exist a positive set of parameters which correspond to Collet-Eckmann maps with 
acims. In [34] it was proved that if S-unimodal map $f$ (i.e. unimodal with negative Schwarzian derivative), and the critical point of multiplicity $d$ satisfies the summability condition with the exponent $1 / d$ then it has an absolutely continuous invariant measure with respect to the Lebesgue measure (recently a stronger result was proved in [9]). The Schwarzian derivative is defined for a $C^{3}$ function $f$ by

$$
S(f)(x):=f^{\prime \prime \prime}(x) / f^{\prime}(x)-\frac{3}{2}\left(f^{\prime \prime}(x) / f^{\prime}(x)\right)^{2},
$$

provided $f^{\prime}(x) \neq 0$. A prototype $S$-unimodal map is given by $z^{2 d}+c$, with $c \in \mathbb{R}$ and $d$ a positive integer.

An absolutely continuous invariant measure provides a useful information about statistical behavior of orbits. We prove the following result for multimodal maps.

Theorem 6 Let $f$ be an analytic function of the unit interval with all periodic points repelling and negative Schwarzian derivative. If $f$ satisfies the summability condition with an exponent

$$
\alpha<\frac{1}{1+\mu_{\max }}
$$

then $f$ has an absolutely continuous invariant measure $\sigma$ with respect to 1-dimensional Lebesgue measure.

Fractal dimensions. We also show that for the Julia sets under considerations are "regular" fractals, in the sense that all possible dimensions coincide.

The Hausdorff dimension of a measure $\nu$ is defined as the infimum of Hausdorff dimensions of its Borel supports:

$$
\operatorname{HDim}(\nu):=\inf \{\operatorname{HDim}(A): \nu(A)=1\} .
$$

For the convenience of the reader we define and discuss basic properties of Hausdorff, Minkowski, and Whitney dimensions HDim, MDim, $\delta_{W h i t}$ in Section 8.

Theorem 7 Suppose that a rational function $F$ satisfies the summability condition with an exponent

$$
\alpha<\frac{p}{\mu_{\max }+p}
$$

where $p$ is any (e.g., maximal) of the quantities in the formula below. Then

$$
\delta_{\text {Poin }}(J)=\delta_{W h i t}(J)=\operatorname{MDim}(J)=\operatorname{HDim}(J)=\operatorname{HypDim}(J)=\operatorname{HDim}(\nu),
$$

where $\nu$ is a unique $\delta_{\text {conf-conformal measure. }}$

Corollary 1.1 Under the hypothesis of Theorem 2, the unique $\delta_{\text {conf-conformal measure } \nu \text { is a }}$ geometric measure. 
A natural question arises: does the claim of Theorem 7 remain valid under any summability condition? This is an interesting question with possible application towards establishing nonatomicity of conformal measures with minimal exponent.

Corollary 1.2 (Strong Ahlfors dichotomy) Suppose that a rational function F satisfies the summability condition with an exponent

$$
\alpha<\frac{2}{\mu_{\max }+2} .
$$

Then the Julia set is either the whole sphere, or its Minkowski dimension is strictly less than 2 .

Unstable rational maps. It is known (by an application of $\lambda$-lemma, see [29]), that the affirmative answer to the dynamical Ahlfors conjecture (Julia set is either the whole sphere or of zero area) in the class of rational functions with $J \neq \hat{\mathbb{C}}$ implies the Fatou conjecture. If $F$ satisfies the summability condition with an exponent $\alpha \leq \frac{2}{2+\mu_{\max }}$ then, by Theorem 1 , the Julia set has zero area, and cannot carry a non-trivial Beltrami differential.

Definition 1.5 We say that a set $J$ is conformally removable if every homeomorphism $\phi$ of $\hat{\mathbb{C}}$ which is holomorphic off $J$, is in fact a Möbius transformation.

For Julia sets, this is a very strong property, which generally does not hold even for hyperbolic rational maps. A counterexample, which is topologically a Cantor set of circles is constructed in $\S 11.8$ of the book [2]. Using a recent work [24], we can establish conformal removability (also called holomorphic removability) of Julia sets for polynomials and Blaschke products satisfying the summability condition.

Theorem 8 If a polynomial $F$ satisfies the summability condition with an exponent

$$
\alpha<\frac{2}{\mu_{\max }+2}
$$

then the Julia set is conformally removable.

More generally, the theorem above holds not only for polynomials, but for rational functions such that the Julia set is a boundary of one of the Fatou components.

The assumption above, that the Julia set coincides with the boundary of one of the Fatou components is essential for conformal removability. A more flexible concept of dynamical removability might hold for all rational Julia sets.

Definition 1.6 We say that a Julia set $J_{F}$ is dynamically removable if every homeomorphism $\phi$ of $\hat{\mathbb{C}}$ which conjugates $(\hat{\mathbb{C}}, F)$ with another rational dynamical system $(\hat{\mathbb{C}}, H)$ and is quasiconformal off $J_{F}$ is globally quasiconformal. 
Theorem 9 If a rational function $F$ satisfies the summability condition with an exponent

$$
\alpha<\frac{1}{\mu_{\max }+1},
$$

then the Julia set is dynamically removable.

Theorem 10 Let a rational function $F$ satisfy the summability condition with an exponent

$$
\alpha<\frac{2}{\mu_{\max }+2} .
$$

Suppose that there is a quasiconformal homeomorphism $\phi$ of $\hat{\mathbb{C}}$ which conjugates rational dynamical systems $(\hat{\mathbb{C}}, F)$ and $(\hat{\mathbb{C}}, H)$.

- If $J \neq \hat{\mathbb{C}}$, then the Beltrami coefficient $\phi_{\mu}$ has to be supported on the Fatou set.

- If $J=\hat{\mathbb{C}}$, then either $\phi$ is a Möbius transformation, or $F$ is double covered by an integral torus endomorphism (i.e. it is a Lattés example). In the latter case the Beltrami coefficient $\mu_{\phi}$ lifts to a constant Beltrami coefficient on the covering torus.

Corollary 1.3 (No invariant line fields) If a rational function $F$ satisfies the summability condition with an exponent

$$
\alpha<\frac{2}{\mu_{\max }+2},
$$

then $J$ carries no invariant line field, except when $F$ is double covered by an integral torus endomorphism.

Compare Corollary 3.18 in [32].

Geometry, local connectivity, and non-wandering continua. If $z^{\ell}+c$ is a unicritical polynomial with locally connected Julia set $J$ then the dynamics on $J$ has a particularly simple representation: it is semi-conjugate to the multiplication by $\ell$ modulo 1 on $[0,1)$. The quest for local connectivity of polynomial Julia sets dates back to the mid-Eighties. Recently, local connectivity was obtained for all real unicritical polynomials $z^{l}+c$ with connected Julia sets, [28], and for Collet-Eckmann polynomials and Blaschke products with connected Julia sets, $[18]$.

The quasihyperbolic distance $\operatorname{dist}_{\mathrm{qh}}(y, z)$ between points $y, z \in \mathcal{F}$ is defined as the infimum of

$$
\operatorname{dist}_{\mathrm{qh}}(y, z):=\inf _{\gamma} \int_{\gamma} \frac{|d \zeta|}{\operatorname{dist}(\zeta, \partial \mathcal{F})},
$$

the infimum taken over all rectifiable curves $\gamma$ joining $y$ and $z$ in $\mathcal{F}$. 
Definition 1.7 We will call a (possibly non-simply-connected) domain $\mathcal{F}$ integrable, if there exists $z_{0} \in \mathcal{F}$ and an integrable function $H: \mathbb{R}_{+} \rightarrow \mathbb{R}_{+}$,

$$
\int_{0}^{\infty} H(r)<\infty
$$

such that $\mathcal{F}$ satisfies the following quasihyperbolic boundary condition:

$$
\operatorname{dist}(z, \partial \mathcal{F}) \leq H\left(\operatorname{dist}_{\mathrm{qh}}\left(z, z_{0}\right)\right)
$$

for any $z \in \mathcal{F}$. The distance dist $(z, \partial \mathcal{F})$ is computed in the spherical metric.

Hölder domains correspond to "exponentially fast integrable" domains with $H(r)=\exp (C-\epsilon r)$. We will show that the Fatou components of rational maps satisfying the summability condition are integrable domains.

A concept of wandering subcompacta of connected Julia sets is directly related to the local connectivity of components of $J$. We say that a compact set $K$ is wandering if for every $m, n>0, F^{n}(K) \cap F^{m}(K)=\emptyset$ whenever $m \neq n$.

Theorem 11 Let $F$ be a rational function which satisfies the summability condition with an exponent

$$
\alpha \leq \frac{1}{\mu_{\max }+1} .
$$

Then every periodic Fatou component $\mathcal{F}$ is an integrable domain. If $F$ has a fully invariant Fatou component then every component of $J$ is locally connected and $F$ does not have wandering continua.

Uniform summability and continuity of dimensions. We also study continuity properties of the Hausdorff dimension of the Julia set as a function of $F: F \mapsto \operatorname{HDim}\left(J_{F}\right)$. To this aim we consider a certain class of perturbations of a rational map $F$ which satisfy the summability condition in a uniform way. Since perturbations usually let critical points escape from the Julia set, we need to take into account critical points of $F$ which do not belong to $J_{F}$.

Given a rational function $F$ and an $\epsilon$-neighborhood $B_{\epsilon}(J)$ of its Julia set $J$ we define for every $c \in C$ rit $\cap B_{\epsilon}(J)$ an escape time

$$
E(\epsilon)=\inf \left\{j \in \mathbb{N}: F^{j}(c) \notin B_{\epsilon}(J)\right\} .
$$

If $F^{j}(c) \in B_{\epsilon}(J)$ for all $j$, we set $E(\epsilon):=\infty$.

Definition 1.8 Let $F$ be a rational function satisfying the summability condition with an exponent $\alpha$. We say that a sequence $\left(F_{i}\right)$ of rational functions converges $S(\alpha)$ - uniformly to $F$ if:

1. $F_{i}$ do not have parabolic periodic orbits and tend to $F$ uniformly on the Riemann sphere, 
2. there exist $M, \epsilon>0$ so that for every $i$ large enough and every $c \in \operatorname{Crit}_{F_{i}} \cap B_{\epsilon}\left(J_{F_{i}}\right)$,

$$
\sum_{j=1}^{E(\epsilon)}\left|\left(F_{i}^{j}\right)^{\prime}(c)\right|^{-\alpha}<M .
$$

3. $\# C r i t_{F}=\#$ Crit $_{F_{i}}$ for $i$ large enough (the critical points are counted without their multiplicities).

The notion of the $S(\alpha)$-uniform convergence involves only these critical points $c$ of $F_{i}$ which "asymptotically" belong to the Julia set $J_{F}$. The $S(\alpha)$-uniform convergence demands $1-1$ correspondence between the critical points of $F$ and $F_{i}$ for large $i$.

Theorem 12 establishes continuity of the Hausdorff (Minkowski) dimension for the $S(\alpha)$ uniform convergence.

Theorem 12 (Continuity of Hausdorff Dimension) Suppose that a rational function $F$ satisfies the summability condition with an exponent

$$
\alpha<\frac{\delta_{\text {Poin }}(J)}{\mu_{\max }+\delta_{\text {Poin }}(J)},
$$

and $\left(F_{i}\right)$ is a sequence of rational functions tending $S(\alpha)$-uniformly to $F$. Then,

$$
\lim _{i \rightarrow \infty} \operatorname{HDim}\left(J_{F_{i}}\right)=\operatorname{HDim}\left(J_{F}\right) .
$$

Unicritical polynomials. Let $\mathcal{M}_{d}$ be the connectedness locus of unicritical polynomials $f_{c}=z^{d}+c$

$$
\mathcal{M}_{d}=\left\{c:\left|f_{c}^{n}(c)\right|<\infty\right\} .
$$

When $d=2, \mathcal{M}_{2}$ is better known as the Mandelbrot set. By Shishikura's theorem [41] it is known that the Hausdorff dimension as a function of $c \in \mathbb{C} \backslash \mathcal{M}_{2}$ does not extend continuously to $\partial M$. Yet typically with respect to the harmonic measure of $\partial \mathcal{M}_{2}$ a continuous extension of $\operatorname{HDim}(\cdot)$ along hyperbolic geodesics is possible.

Definition 1.9 A closure $\Gamma(c)$ of a hyperbolic geodesic in $\mathbb{C} \backslash \mathcal{M}_{d}$ which contains $\infty$ and a point $c \in \partial M_{d}$ is called an external ray. If $\Gamma(c) \cap \partial \mathcal{M}_{d}=\{c\}$ then we say that $\Gamma(c)$ terminates at $c$.

We use properties of the $S(\alpha)$-convergence with $\alpha<1 /(1+d)$, and the results of $[20,42]$ to deduce the following theorem.

Theorem 13 For almost all c from $\partial \mathcal{M}_{d}$ with respect to the harmonic measure, we have

$$
\lim _{\Gamma(c) \ni c^{\prime} \rightarrow c} \operatorname{HDim}\left(J_{c^{\prime}}\right)=\operatorname{HDim}\left(J_{c}\right) .
$$


Radial continuity of Hausdorff dimension for postcritically finite quadratic polynomials was established in [33]. The set of postcritically finite polynomials is of zero harmonic measure, $[20,42]$.

Another consequence of our estimates and [20,42] is a conformal analogue of Jakobson and Benedicks-Carleson's theorem $[23,3,4]$. Let $f_{c}(z)=z^{d}+c$ and suppose that $f_{c}$ has a geometric measure. We call a probabilistic measure $\mu$, supported on the Julia set of $f_{c}$, a Sinai-RuelleBowen, or SRB for short, measure if it is a weak accumulation point of measures $\mu_{n}$ equally distributed along the orbits $x, f_{c}(x), \ldots, f_{c}^{n}(x)$ for $x$ in a positive geometric measure set.

Theorem 14 For almost all $c \in \partial \mathcal{M}_{d}$ with respect to the harmonic measure,

1. there exists a unique geometric measure $\nu_{c}$ of $z^{d}+c$ which is a weak limit of the normalized Hausdorff measures of $J_{c^{\prime}}, c^{\prime} \in \Gamma(c)$.

2. $\nu_{c}$ is ergodic and non-atomic,

3. $\operatorname{HDim}\left(\nu_{c}\right)=\operatorname{HDim}(J)$,

4. $z^{d}+c$ has an invariant SRB measure with a positive Lyapunov exponent which is equivalent to the geometric measure $\nu_{c}$.

Acknowledgments. The authors are grateful to the referee for carefully reading our manuscript and contributing numerous suggestions, which greatly improved the exposition.

Stanislav Smirnov would like to thank Zoltan Balogh, Chris Bishop, and Pekka Koskela for many useful discussions.

Notation and Conventions. We will write $A \lesssim B$ whenever $A \leq C B$ with some absolute (but depending on the equation) constant $C$. If $A \leq C B$ and $B \leq C A$ then we write $A \asymp B$. We will denote a ball of radius $R$ around $z$ by $B_{R}(z)$. We adopt the convention that $\sum_{n}\left(\omega_{n}\right)^{-\infty}<\infty$ means that the sequence $\omega_{n}$ tends to $\infty$ as $n \rightarrow \infty$.

For simplicity and readers convenience we will write all the distortion estimates for the planar metric, when Koebe distortion theorem has a more familiar formulation (Lemma 2.1). The estimates remain valid in the case of spherical metric, with an appropriate version of Koebe distortion theorem (which differs only by a multiplicative constant, since we work with the scales smaller than some very small $R$ ).

Another general convention is following: we call $F^{-n}(z), \ldots, z$ a sequence of preimages of $z$ by $F$ if for every $1 \leq j \leq n$,

$$
F\left(F^{-j}\right)(z)=F^{-j+1}(z)
$$




\section{Part I}

\section{Poincaré series, induced hyperbolicity, invariant measures}

\section{Expansion along orbits}

Our goal is to estimate the derivative of $F^{n}$ at $z$ in terms of the summability condition and the injectivity radius of the corresponding inverse branch $F^{-n}$ at $F^{n}(z)$. This is attained by decomposing backward orbits into pieces which either closely follow the critical orbits or stay away from the critical points at a definite distance. We provide also a technical introduction to the theory of the Poincaré series for rational maps.

Proposition 2.1 can be regarded as a conditional version of induced hyperbolicity. In applications, we will use a stronger statement (the Main Lemma), which contains more technically detailed assertions. The proof of the Main Lemma will supply a procedure of decomposing any orbit into blocks of three different types, defined rigorously in Subsection 2.4. We will show that the derivative along each block of a given type is expansive up to an error term which is a function of a few dynamically defined parameters. The main difficulty in proving Proposition 2.1 is a possibility of accumulation of error terms. We will prove that due to cancellations, the expansion prevails.

After initial preparations in Section 2, the proof of Proposition 2.1 will be concluded in Section 3.

Proposition 2.1 Let a rational function $F$ satisfy the summability condition with an exponent $\alpha \leq 1$. There exist $\epsilon>0$ and a positive sequence $\left\{\omega_{n}\right\}$ summable with an exponent $-\beta:=$ $-\frac{\mu_{\max } \alpha}{1-\alpha}$, meaning $\sum_{n}\left(\omega_{n}\right)^{-\beta}<\infty$, so that for every point $z$ from $\epsilon$-neighborhood of the Julia set and every univalent branch of $F^{-n}$ defined on the ball $B_{\Delta}(z)$ with $\Delta<\epsilon$,

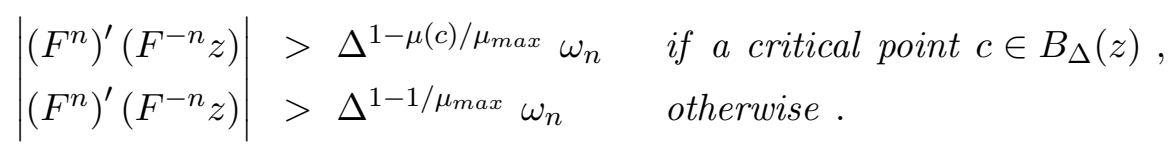

Remark 2.1 The statement above is well-formulated even when when $\alpha=1$, if we recall the convention that $\sum_{n}\left(\omega_{n}\right)^{-\infty}<\infty$ means that the sequence $\omega_{n}$ tends to $\infty$ as $n \rightarrow \infty$.

Remark 2.2 In the proof we actually obtain a specific form of $\omega_{n}$ in terms of $\left\{\sigma_{n}\right\}$ and Mañé's lemma.

Corollary 2.1 Suppose that the assumptions of Proposition 2.1 are satisfied. Then there is $\epsilon>0$ such that for any point $z$ at a distance $\Delta<\epsilon$ from the Julia set we have

$$
\left|\left(F^{n}\right)^{\prime}\left(F^{-n} z\right)\right| \geq \Delta^{1-1 / \mu_{\max }} \omega_{n},
$$

where $\omega_{n}$ is given by Proposition 2.1. 
Corollary 2.2 If $F$ satisfies the summability condition with an exponent $\alpha \leq 1$ then $F$ has neither Siegel disks nor Herman rings.

Proof: If $z$ belongs to an elliptic Fatou component $\mathcal{F}$ (Siegel disk or Herman ring) then for every preimage $F^{-n} z \in \mathcal{F},\left|\left(F^{n}\right)^{\prime}\left(F^{-n} z\right)\right| \asymp 1$. This contradicts Proposition 2.1 since $\lim _{n \rightarrow \infty} \omega_{n}=\infty$.

\subsection{Preliminaries}

Shrinking neighborhoods. To control the distortion, we will use the method of shrinking neighborhoods, introduced in [37] (see also [18]). Suppose that $\sum_{n=1}^{\infty} \delta_{n}<1 / 2$ and $\delta_{n}>0$ for every positive integer $n$. Set $\Delta_{n}:=\prod_{k \leq n}\left(1-\delta_{k}\right)$. Let $B_{r}$ be a ball of radius $r$ around a point $z$ and $\left\{F^{-n} z\right\}$ be a sequence of preimages of $z$. We define $U_{n}$ and $U_{n}^{\prime}$ as the connected components of $F^{-n} B_{r \Delta_{n}}$ and $F^{-n} B_{r \Delta_{n+1}}$, respectively, which contain $F^{-n} z$. Clearly,

$$
F U_{n+1}=U_{n}^{\prime} \subset U_{n} \text {. }
$$

If $U_{k}$, for $1 \leq k \leq n$, do not contain critical points then distortion of $F^{n}: U_{n}^{\prime} \rightarrow B_{r \Delta_{n+1}}$ is bounded (Koebe distortion lemma below) by a power of $\frac{1}{\delta_{n+1}}$, multiplied by an absolute constant.

Since $\sum_{n} \delta_{n}<\frac{1}{2}$, one also has $\prod_{n}\left(1-\delta_{n}\right)>\frac{1}{2}$, and hence always $B_{r / 2} \subset B_{r \Delta_{n}}$.

The Koebe distortion lemma. We will use the following version of the Koebe distortion lemma.

Lemma 2.1 Let $f: B \rightarrow \mathbb{C}$ be a univalent map from the unit disk into the complex plane. Then the image $f(B)$ contains a ball of radius $\frac{1}{4}\left|f^{\prime}(0)\right|$ around $f(0)$. Moreover, for every $z \in B$ we have that

$$
\frac{(1-|z|)}{(1+|z|)^{3}} \leq \frac{\left|f^{\prime}(z)\right|}{\left|f^{\prime}(0)\right|} \leq \frac{(1+|z|)}{(1-|z|)^{3}}
$$

and

$$
|f(z)-f(0)| \leq\left|f^{\prime}(z)\right| \frac{|z|(1+|z|)}{1-|z|} .
$$

Proof: The first statement is Corollary 1.4, and the next inequality is Theorem 1.3 in [35].

Let $M$ be a Möbius automorphism of the unit disk which maps 0 onto $z$ and $-z$ onto 0 . By Theorem 1.3 in [35] applied to $f \circ M$, we have that

$$
|f \circ M(-z)-f \circ M(0)| \leq\left|(f \circ M)^{\prime}(0)\right| \frac{|z|}{(1-|z|)^{2}} .
$$

Since $M^{\prime}(0)=1-|z|^{2}$, the last claim of the lemma follows. 


\subsection{Technical sequences}

Suppose that $F$ is a rational function which satisfies the summability condition with an exponent $\alpha$. To simplify calculations we will introduce three positive sequences $\left\{\alpha_{n}\right\},\left\{\gamma_{n}>1\right\},\left\{\delta_{n}\right\}$ so that the growth of the derivative of $F^{n}$ will be expressed in terms of $\gamma_{n}$, the corresponding distortion will be majorized by $\delta_{n}$, and the constants will be controlled through $\alpha_{n}$.

Lemma 2.2 If a sequence $\left\{1 / \sigma_{n}\right\}$ is summable with an exponent $\alpha \leq 1: \sum\left(\sigma_{n}\right)^{-\alpha}<\infty$, then there exist three positive sequences $\left\{\alpha_{n}\right\},\left\{\gamma_{n}\right\},\left\{\delta_{n}\right\}$, such that

$$
\begin{aligned}
\lim _{n \rightarrow \infty} \alpha_{n} & =\infty \\
\sum_{n}\left(\gamma_{n}\right)^{-\beta} & <1 /\left(16 \operatorname{deg} F \cdot \mu_{\max }\right), \quad \beta:=\frac{\mu_{\max } \alpha}{1-\alpha} \\
\sum_{n} \delta_{n} & <1 / 2
\end{aligned}
$$

and

$$
\sigma_{n} \geq\left(\alpha_{n}\right)^{2}\left(\gamma_{n}\right)^{\mu_{\max }} / \delta_{n}
$$

Proof: Suppose that $\alpha<1$. We set

$$
\delta_{n}^{\prime}:=\left(\sigma_{n}\right)^{-\alpha}, \quad \gamma_{n}^{\prime \prime}:=\left(\sigma_{n}\right)^{(1-\alpha) / \mu_{\max }},
$$

and observe that $\sigma_{n}=\left(\gamma_{n}^{\prime \prime}\right)^{\mu_{\max }} / \delta_{n}^{\prime}, \sum_{n} \delta_{n}^{\prime}<\infty, \sum_{n}\left(\gamma_{n}^{\prime \prime}\right)^{-\beta}<\infty$. It is an easy exercise, that there is a sequence $\left\{\alpha_{n}^{\prime}\right\}, \lim _{n \rightarrow \infty} \alpha_{n}^{\prime}=\infty$, such that $\left\{\gamma_{n}^{\prime}\right\}$ defined by

$$
\gamma_{n}^{\prime}:=\left(\sigma_{n}\right)^{(1-\alpha) / \mu_{\max }} /\left(\alpha_{n}^{\prime}\right)^{-2 / \mu_{\max }}
$$

is still summable with an exponent $-\beta$. Evidently, $\sigma_{n}=\left(\alpha_{n}^{\prime}\right)^{2}\left(\gamma_{n}^{\prime}\right)^{\mu_{\max }} / \delta_{n}^{\prime}$. Now, choose suitable constants $C_{\gamma}, C_{\delta}$ so that $\left\{\gamma_{n}\right\}:=\left\{C_{\gamma} \gamma_{n}^{\prime}\right\}$ and $\left\{\delta_{n}\right\}:=\left\{C_{\delta} \delta_{n}^{\prime}\right\}$ satisfy

$$
\sum_{n} \delta_{n}<1 / 2, \quad \sum_{n} \gamma_{n}^{-\beta}<m
$$

Set $C_{\alpha}:=\sqrt{C_{\delta} / C_{\gamma}^{\mu_{\max }}}$ and let $\alpha_{n}:=C_{\alpha} \alpha_{n}^{\prime}$. Then

$$
\lim _{n \rightarrow \infty} \alpha_{n}=\infty, \quad \sigma_{n} \geq\left(\alpha_{n}\right)^{2}\left(\gamma_{n}\right)^{\mu_{\max }} / \delta_{n} .
$$

The case of $\alpha=1$ can be treated similarly.

\subsection{Constants and scales}

A scale around critical points is given in terms of fixed numbers $R^{\prime} \ll R \ll 1$. We will refer to objects which stay away from the critical points and are comparable with $R^{\prime}$ as the objects of the large scale. The proper choice of $R$ 's is one of the most important elements in the analysis of expansion along pieces of orbits.

We impose the following conditions on $R$ and $R^{\prime}$ (note that sup $\left|F^{\prime}\right|<\infty$, since we use the spherical metric): 
(i) Any two critical points are at least $100 R$ apart and there exists a constant $M>1$ which depends only on $R$ and so that if $\operatorname{dist}(F y, F($ Crit $))<\left(1+\sup \left|F^{\prime}\right|\right) R<1$ or $\operatorname{dist}(y$, Crit $)<5 R^{1 / \mu_{\max }}$ then

$$
\begin{gathered}
1 / M<\left|F^{\prime}(y)\right| / \operatorname{dist}(y, c)^{\mu(c)-1}<M, \\
1 / M<\operatorname{dist}(F y, F c) / \operatorname{dist}(y, c)^{\mu(c)}<M .
\end{gathered}
$$

(ii) $\quad R$ is so small that the first return time of the critical points in the Julia set to $\bigcup F^{-1} B_{R}\left(F c_{i}\right)$ is greater than a constant $\tau$ chosen so that $\alpha_{k}>16^{\mu_{\max }} M^{2}>1$, for $k \geq \tau$.

(iii) $R^{\prime}$ is so small that $16 R^{\prime} \sup \left|F^{\prime}\right| / \inf _{n}\left(\alpha_{n}\right)^{2} \leq R M^{-1}$.

(iv) $R^{\prime}$ is so small that there are no critical points in the $2 R^{\prime}$-neighborhood of the Julia set inside the Fatou set.

Dictionary of constants. For the sake of clarity we list here other constants and indicate their mutual dependence and places of introduction.

$$
\begin{aligned}
& L=\operatorname{const}\left(R^{\prime}, q\right), K, R_{2 t}=\operatorname{const}\left(L, R^{\prime}\right), \text { and } C(p)=\operatorname{const}\left(L, R^{\prime}, p\right) \text { in Lemma } 2.4, \\
& C_{3 t}=\operatorname{const}\left(R_{2 t}\right) \text { in Lemma } 2.5, \\
& L^{\prime \prime}=\operatorname{const}\left(C_{3 t}, R_{2 t}\right), L^{\prime}=\operatorname{const}\left(L^{\prime \prime}\right) \text { in Subsection } 3.2 .
\end{aligned}
$$

\subsection{Types of orbits}

The general scheme of decomposing backward orbits into "expansive" blocks was introduced in [18] for Collet-Eckmann dynamics. Despite many similarities, our setting is substantially less hyperbolic than that given by the Collet-Eckmann condition. Though it is not possible to use directly the estimates of [18] and new results are needed, the strategy to capture expansion is similar: we classify pieces of orbits, depending on whether they are close to critical points or not, and derive expansion estimates. We will obtain three different types of estimates, which when combined will yield "expansion" of the derivative along any orbit.

First type. Our objective is to estimate expansion along pieces of the backward orbit which "join" two critical points, i.e. there is a disk in a vicinity of the first critical point which can be pulled back conformally along the orbit until its boundary hits the second critical point.

The formulation of Lemma 2.3 has to encompass the possibility of critical points with different multiplicities and hence it does not guarantee immediate expansion.

Definition 2.1 A sequence $F^{-n}(z), \cdots, F^{-1}(z), z$ of preimages of $z$ is of the first type with respect to critical points $c_{1}$ and $c_{2}$ if there exists a radius $r<2 R^{\prime}$ such that

1) Shrinking neighborhoods $U_{k}$ for $B_{r}(z), 1 \leq k<n$, avoid critical points, 
2) The critical point $c_{2} \in \partial U_{n}$,

3) The critical point $c_{1} \in F^{-1} B_{R}(F z)$.

Note that given the sequence of preimages, the radius $r$ is uniquely determined by the condition 2 ). To simplify notation set $\mu_{i}:=\mu\left(c_{i}\right), d_{2}:=\operatorname{dist}\left(F^{-n} z, c_{2}\right)$, and $d_{1}:=\operatorname{dist}\left(z, c_{1}\right)$. Let $r_{2}^{\prime}$ be the maximal radius so that $B_{r_{2}^{\prime}}\left(F^{-n} z\right) \subset F^{-n}\left(B_{r / 2}(z)\right)$. For consistency, put $r_{1}:=r$.

Lemma 2.3 For any sequence $y=F^{-n}(z), \cdots, F^{-1}(z), z$ of preimages of the first type and any $\mu \leq \mu_{\max }$ we have
1) $\left|\left(F^{n}\right)^{\prime}(y)\right|^{\mu}>\alpha_{n}\left(\gamma_{n}\right)^{\mu_{\max }} 2^{\mu_{\max }} \frac{\left(d_{2}\right)^{\mu_{2}-1}}{\left(r_{2}^{\prime}\right)^{\mu^{-1}}} \frac{\left(r_{1}\right)^{\mu-1}}{\left(r_{1}+d_{1}\right)^{\mu_{1}-1}}$,
2) $\left(d_{2}\right)^{\mu_{2}}<\left(\max \left(r_{1}, d_{1}\right)\right)^{\mu_{1}}\left(\gamma_{n}\right)^{-\mu_{\max }}$,
3) $\operatorname{dist}\left(F y, F c_{2}\right) \leq R$.

Proof: By the construction the map $F^{-(n-1)}: B_{\Delta_{n-1} r_{1}}(z) \rightarrow U_{n-1}$ is conformal. The Koebe distortion Lemma 2.1 implies that

$$
\begin{aligned}
\operatorname{dist}\left(F y, F c_{2}\right) & \leq \frac{\left(1-\delta_{n}\right)\left(2-\delta_{n}\right)}{\delta_{n}} \Delta_{n-1} r_{1}\left|\left(F^{n-1}\right)^{\prime}\left(F c_{2}\right)\right|^{-1} \\
& \leq \frac{2 R^{\prime}}{\delta_{n}} \sup \left|F^{\prime}\right|\left|\left(F^{n}\right)^{\prime}\left(F c_{2}\right)\right|^{-1} \\
& \leq \frac{2 R^{\prime}}{\delta_{n}} \sup \left|F^{\prime}\right| \delta_{n} /\left(\alpha_{n}\right)^{2} \leq R
\end{aligned}
$$

and the third inequality follows by the choice of $R^{\prime}$ (see (iii)).

We prove the first inequality. The condition 2) of Definition 2.1 implies that $F^{n} c_{2} \in \partial B_{r} \Delta_{n}$. Hence

$$
\operatorname{dist}\left(F^{n} c_{2}, c_{1}\right) \leq \operatorname{dist}\left(F^{n} c_{2}, z\right)+\operatorname{dist}\left(z, c_{1}\right) \leq r_{1}+d_{1} .
$$

Since dist $\left(F^{n+1} c_{2}, F c_{1}\right)$ is small (less than $\left.\left(1+\sup \left|F^{\prime}\right|\right) R\right)$, by the choice of $R$ we have $\left|F^{\prime}\left(F^{n} c_{2}\right)\right| \leq M \operatorname{dist}\left(F^{n} c_{2}, c_{1}\right)^{\mu_{1}-1}$. Thus

$$
\left|\left(F^{n-1}\right)^{\prime}\left(F c_{2}\right)\right| \geq\left|\left(F^{n}\right)^{\prime}\left(F c_{2}\right)\right| \frac{1}{M \operatorname{dist}\left(F^{n} c_{2}, c_{1}\right)^{\mu_{1}-1}} \geq \frac{\sigma_{n}}{M\left(r_{1}+d_{1}\right)^{\mu_{1}-1}} .
$$

Considering the conformal map $F^{-(n-1)}: B_{r \Delta_{n-1}}(z) \rightarrow U_{n-1}$, by the Koebe distortion lemma 2.1 we obtain that

$$
\left|\left(F^{-(n-1)}\right)^{\prime}\left(F^{n} c_{2}\right)\right| \geq \frac{\delta_{n}}{\left(2-\delta_{n}\right)^{3}}\left|\left(F^{-(n-1)}\right)^{\prime}(z)\right|
$$

and therefore

$$
\left|\left(F^{n}\right)^{\prime}(y)\right| \geq \frac{\delta_{n}}{8 M}\left|\left(F^{n-1}\right)^{\prime}\left(F c_{2}\right)\right| \operatorname{dist}\left(y, c_{2}\right)^{\mu_{2}-1}
$$


Together with the estimate (6) this yields

$$
\left|\left(F^{n}\right)^{\prime}(y)\right| \geq \frac{\delta_{n}}{8 M^{2}} \sigma_{n} \frac{\left(d_{2}\right)^{\mu_{2}-1}}{\left(r_{1}+d_{1}\right)^{\mu_{1}-1}} .
$$

By the Koebe lemma, the image of the map $F^{-n}: B_{r_{1} / 2}(z) \rightarrow U_{n}$ contains a ball of radius $\frac{1}{8} r_{1}\left|\left(F^{n}\right)^{\prime}(y)\right|^{-1}$ and the center $y$. Hence

$$
\left|\left(F^{n}\right)^{\prime}(y)\right| \geq \frac{r_{1}}{8 r_{2}^{\prime}} .
$$

Combining the above estimate raised to the power $(\mu-1)$ with the previous one we obtain

$$
\begin{aligned}
\left|\left(F^{n}\right)^{\prime}(y)\right|^{\mu} & \geq \frac{\delta_{n}}{8^{\mu} M^{2}} \sigma_{n} \frac{\left(d_{2}\right)^{\mu_{2}-1}}{\left(r_{2}^{\prime}\right)^{\mu-1}} \frac{\left(r_{1}\right)^{\mu-1}}{\left(r_{1}+d_{1}\right)^{\mu_{1}-1}} \\
& =\frac{\delta_{n}}{8^{\mu} M^{2}}\left(\delta_{n}\right)^{-1}\left(\alpha_{n}\right)^{2}\left(\gamma_{n}\right)^{\mu_{\max }} \frac{\left(d_{2}\right)^{\mu_{2}-1}}{\left(r_{2}^{\prime}\right)^{\mu-1}} \frac{\left(r_{1}\right)^{\mu-1}}{\left(r_{1}+d_{1}\right)^{\mu_{1}-1}} \\
& >\alpha_{n}\left(\gamma_{n}\right)^{\mu_{\max }} 2^{\mu_{\max }} \frac{\left(d_{2}\right)^{\mu_{2}-1}}{\left(r_{2}^{\prime}\right)^{\mu-1}} \frac{\left(r_{1}\right)^{\mu-1}}{\left(r_{1}+d_{1}\right)^{\mu_{1}-1}} .
\end{aligned}
$$

The last inequality holds since by the choice of $R, n \geq \tau$.

Note that using the inequality (6) we can modify the estimate (5) by writing

$$
\begin{aligned}
\left(d_{2}\right)^{\mu_{2}} & \leq M \operatorname{dist}(F y, F c) \\
& \leq \frac{\left(1-\delta_{n}\right)\left(2-\delta_{n}\right)}{\delta_{n}} \Delta_{n-1} r_{1}\left|\left(F^{n-1}\right)^{\prime}\left(F c_{2}\right)\right|^{-1} \\
& \leq M \frac{2 r_{1}}{\delta_{n}} \cdot \frac{M\left(r_{1}+d_{1}\right)^{\mu_{1}-1}}{\sigma_{n}} \\
& \leq 2^{\mu_{\max }} M^{2}\left(\max \left(r_{1}, d_{1}\right)\right)^{\mu_{1}}\left(\alpha_{n}\right)^{-2}\left(\gamma_{n}\right)^{-\mu_{\max }} \\
& =2^{\mu_{\max }} M^{2}\left(\alpha_{n}\right)^{-2}\left(\max \left(r_{1}, d_{1}\right)\right)^{\mu_{1}}\left(\gamma_{n}\right)^{-\mu_{\max }}<\left(\max \left(r_{1}, d_{1}\right)\right)^{\mu_{1}}\left(\gamma_{n}\right)^{-\mu_{\max }}
\end{aligned}
$$

which completes the proof of the second inequality.

Second type. A piece of a backward orbit is of the second type if there exists a neighborhood of size $R^{\prime}$ which can be pulled back univalently along the backward orbit. Type two preimages yield expansion along pieces of orbits of a uniformly bounded length. In this "uniformly bounded" setting, type 2 corresponds to pieces of backward orbits which stay at a definite distance from the critical points.

Definition 2.2 Let $\operatorname{dist}\left(z, J_{F}\right) \leq R^{\prime} / 2$. A sequence $F^{-n}(z), \cdots, F^{-1}(z)$, $z$ of preimages of $z$ is of the second type if the ball $B_{R^{\prime}}(z)$ can be pulled back univalently along it. 
Lemma 2.4 Let $\mathbf{I I}(z)$ be the set of all preimages of the second type of some point $z$. Denote $n(y):=n$ if $F^{n} y=z$.

1. There exists a constant $L>0$ so that the following holds:

$$
\inf _{y \in \mathbf{I I}(z), n(y) \geq L}\left|\left(F^{n}\right)^{\prime}(y)\right|>6 .
$$

2. If the Poincaré series $\Sigma_{q}(v)$ converges for some point $v$, then $L$ can be chosen so that

$$
\sum_{y \in \mathbf{I I}(z), n(y) \geq L}\left|\left(F^{n}\right)^{\prime}(y)\right|^{-q}<\frac{1}{36} .
$$

3. Once $L$ is chosen there exist positive constants $K$ and $C(q)$ such that

$$
\begin{array}{ll}
\sum_{y \in \mathbf{I I}(z), n(y) \leq L}\left|\left(F^{n}\right)^{\prime}(y)\right|^{-q}<C(q), & \text { and } \\
\left|\left(F^{n}\right)^{\prime}(y)\right|>K & \text { for every } y \in \mathbf{I I}(z), n(y) \leq L .
\end{array}
$$

4. Once $L$ is chosen there is a positive $R_{2 t}$ such that for any point $z$ and its second type preimage $y=F^{-L} z$ of order $L$ we have

$$
B_{2 R_{2 t}}(y) \subset F^{-L}\left(B_{R^{\prime}}(z)\right) .
$$

Proof: The proof of the first part is standard and follows from the compactness argument. Suppose that the claim does not hold. Then there is an infinite collection of sequences of the second type

$$
F^{-n_{i}}\left(z_{i}\right), \ldots F^{-1}\left(z_{i}\right), z_{i}
$$

such that $n_{i} \rightarrow \infty$ and $\left|\left(F^{n_{i}}\right)^{\prime}\left(F^{-n_{i}}\left(z_{i}\right)\right)\right| \leq 6$. Consider the preimages $F^{-n_{i}}\left(B_{R^{\prime} / 2}\left(z_{i}^{\prime}\right)\right) \ni$ $F^{-n_{i}}\left(z_{i}\right)$, where $z_{i}^{\prime}$ is the closest point to $z_{i}$ in $J_{F}$. Without loss of generality we can assume that $R^{\prime} \ll \operatorname{diam} J_{F}$. By the Koebe distortion lemma 2.1, any of these preimages contains a round ball around $F^{-n_{i}}\left(z_{i}^{\prime}\right)$ of the radius larger than $\eta:=R^{\prime} /(8 \cdot 6)$. Let $y$ be an accumulation point of the sequence $F^{-n_{i}}\left(z_{i}^{\prime}\right) \in J_{F}$. By the construction, there is an increasing subsequence $\left\{k_{j}\right\}$ of the sequence $\left\{n_{j}\right\}$ such that the images of $B_{\eta / 2}(y)$ under $F^{k_{j}}$ are contained in $B_{R^{\prime}}(z) \not \supset J_{F}$ and we arrived at a contradiction, since $y \in J_{F}$ and the Julia set has the eventually onto property (see Theorem 1 in [10]).

To prove the second part, we recall again that if the Poincaré series for $v$ converges then $v$ must be a non-exceptional point, i.e. with preimages dense in the Julia set. We can fix finitely many of them, say $v_{1}, \ldots, v_{n}$, so that they are $R^{\prime} / 4$-dense in $J$ and their Poincaré series will also converge. Then for any point $z$ with $\operatorname{dist}\left(z, J_{F}\right)<R^{\prime} / 2$ there is a point $v_{j} \in B_{3 R^{\prime} / 4}(z)$. By the Koebe distortion lemma 2.1, we can write

$$
\sum_{y \in \mathbf{I I}(z)}\left|\left(F^{n}\right)^{\prime}(y)\right|^{-q} \lesssim \Sigma_{q}\left(v_{j}\right) \leq \max _{j} \Sigma_{q}\left(v_{j}\right) \lesssim \Sigma_{q}(v)<\infty,
$$


and after a proper choice of $L$ the second inequality of the lemma follows.

For the third part, we use the Koebe distortion lemma 2.1,

$$
1 \gtrsim \operatorname{diam}\left(F^{-n} B_{R^{\prime}}(z)\right) \geq \frac{1}{4} R^{\prime}\left|\left(F^{-n}\right)^{\prime}(z)\right|=\frac{1}{4} R^{\prime}\left|\left(F^{n}\right)^{\prime}\left(F^{-n}(z)\right)\right| .
$$

Both statements easily follow.

The fourth part is an immediate consequence of the Koebe distortion lemma.

Third type. The third type gives more leeway in choosing blocks than the types 1 and 2 . In [18] a more restrictive approach was used. Blocks of type 3 connect the large scale with critical points.

Definition 2.3 A sequence $F^{-n}(z), \cdots, F^{-1}(z), z$ of preimages of $z$ is of the third type with respect to the critical point $c_{2}$ if there exists a radius $r<2 R^{\prime}$ such that

1) Shrinking neighborhoods $U_{k}$ for $B_{r}(z), 1 \leq k<n$, avoid critical points,

2) The critical point $c_{2} \in \partial U_{n}$.

Note that given the sequence of preimages, the radius $r$ is uniquely determined by the condition 2).

The next lemma estimates the expansion along the third type preimages. To simplify notation set $\mu_{2}:=\mu\left(c_{2}\right), d_{2}:=\operatorname{dist}\left(F^{-n} z, c_{2}\right)$. Let $r_{2}^{\prime}$ be the maximal radius so that $B_{r_{2}^{\prime}}\left(F^{-n} z\right) \subset F^{-n}\left(B_{r / 2}(z)\right)$. For consistency, put $r_{1}:=r$.

Lemma 2.5 There exists $C_{3 t}>0$ such that for any sequence of preimages of the third type $F^{-n}(z), \cdots, F^{-1}(z), z$ and any $\mu \leq \mu_{\max }$ we have

1) $\left|\left(F^{n}\right)^{\prime}(y)\right|^{\mu}>C_{3 t} \alpha_{n}\left(\gamma_{n}\right)^{\mu_{\max }} \frac{\left(d_{2}\right)^{\mu_{2}-1}}{\left(r_{2}^{\prime}\right)^{\mu-1}}\left(r_{1}\right)^{\mu-1}$,

2) $\operatorname{dist}\left(F y, F c_{2}\right) \leq R$,

If the sequence of the third type is preceded by a sequence of the second type of length $L$ then we can substitute $R_{2 t}$ for $r_{1}$ in the estimate 1 ).

Proof: The proof of the second inequality follows from (5). Indeed, we did not use there the existence of a critical point close to $z$, so the proof works for the third type of preimages. Equation (5) implies the following estimate

$$
\left(d_{2}\right)^{\mu_{2}} \leq M \operatorname{dist}(F y, F c) \leq M 2 R^{\prime} \sup \left|F^{\prime}\right| /\left(\alpha_{n}\right)^{2},
$$

and therefore

$$
\operatorname{dist}(y, c) \rightarrow 0, \quad \text { as } n \rightarrow \infty
$$


The inequalities (7) and (8) from the proof of Lemma 2.3 are valid also for the third type preimages. So using the same notation, we can write

$$
\begin{aligned}
\left|\left(F^{n}\right)^{\prime}(y)\right| & \geq \frac{\delta_{n}}{8 M}\left|\left(F^{n-1}\right)^{\prime}\left(F c_{2}\right)\right|\left(d_{2}\right)^{\mu_{2}-1} \\
& \geq \frac{\delta_{n}}{8 M} \frac{\sigma_{n}}{\sup \left|F^{\prime}\right|}\left(d_{2}\right)^{\mu_{2}-1}
\end{aligned}
$$

and

$$
\left|\left(F^{n}\right)^{\prime}(y)\right| \geq \frac{r_{1}}{8 r_{2}^{\prime}} .
$$

Combining these estimates we conclude that

$$
\begin{aligned}
\left|\left(F^{n}\right)^{\prime}(y)\right|^{\mu} & \geq \frac{\delta_{n}}{8^{\mu} M} \frac{\sigma_{n}}{\sup \left|F^{\prime}\right|} \frac{\left(d_{2}\right)^{\mu_{2}-1}}{\left(r_{2}^{\prime}\right)^{\mu-1}}\left(r_{1}\right)^{\mu-1} \\
& >C_{3 t} \alpha_{n}\left(\gamma_{n}\right)^{\mu_{\max }} \frac{\left(d_{2}\right)^{\mu_{2}-1}}{\left(r_{2}^{\prime}\right)^{\mu-1}}\left(r_{1}\right)^{\mu-1}
\end{aligned}
$$

where

$$
C_{3 t}:=\inf _{n}\left\{\alpha_{n}\left(8^{\mu_{\max }} M \sup \left|F^{\prime}\right|\right)^{-1}\right\}>0 \text {. }
$$

It remains to observe that the last assertion of the lemma is true since if a sequence of the third type is preceded by a sequence of the second type of length $L$ then $r_{1}>R_{2 t}$ by Lemma 2.4 .

\section{Specification of orbits}

We will estimate expansion along the backward orbits by decomposing them into blocks of different types described in Section 2.

Lemma 2.4, which governs the expansion in the large scale, was stated in the proximity of the Julia set, and to apply it we will need the following Lemma, which holds in the absence of parabolic points (see Lemma 5 in [18]).

Lemma 3.1 There exists $\epsilon>0$ such that the backward orbit of any $z$ in the $\epsilon$-neighborhood of the Julia set stays in the $R^{\prime} / 2$-neighborhood of the Julia set.

This means that the assertions of Lemma 2.4 are valid for type 2 preimages $F^{-n}(z), n>0$, provided $z$ belongs to an $\epsilon$-neighborhood of the Julia set.

Definition 3.1 We say that a backward orbit $y=F^{-n}(z), \ldots, z$ is decomposed into a sequence of blocks if there exists an increasing sequence of integers $0=n_{0}<\ldots<n_{k}=n$ so that for every $i=0, \ldots, k-1$ the orbit $F^{-n_{i+1}}(z), \ldots, F^{-n_{i}}(z)$ is of type 1,2 , or 3 . Given a pair of integers $0 \leq r<l \leq n$, we say that a subsequence $F^{-n_{l}}(y), \ldots, F^{-n_{r}}(y)$ yields expansion $M$ if

$$
\left|\left(F^{n_{l}-n_{r-1}}\right)^{\prime}(y)\right| \geq M .
$$

The point $F^{-n_{l}}(z)$ is called a terminal point of the subsequence. 
Recall that $\sigma_{n}:=\min _{c \in \operatorname{Crit}}\left\{\left|\left(F^{n}\right)^{\prime}(F c)\right|\right\}$ was represented as the product $\left(\alpha_{n}\right)^{2}\left(\gamma_{n}\right)^{\mu_{\max }} / \delta_{n}$. The sequence $\left\{\delta_{n}\right\}$ will majorate the distortion in the shrinking neighborhoods construction, $\left\{\alpha_{n}\right\}$ will swallow all remaining constants, and $\left\{\gamma_{n}\right\}$ will provide the desired expansion.

Lemma 3.2 (Main Lemma) Let $\epsilon$ be supplied by Lemma 3.1. Assume that a rational function $F$ satisfies the summability condition with an exponent $\alpha \leq 1$ and set $\beta=\mu_{\max } \alpha /(1-\alpha)$. Suppose that a point $z$ belongs to $\epsilon$-neighborhood of the Julia set $J$ and a ball $B_{\Delta}(z)$ can be pulled back univalently by a branch of $F^{-N}$. We claim that there exist positive constants $L^{\prime}>L, K$ independent of $z, \Delta$, and $\epsilon$ such that the sequence $F^{-N}(z), \ldots, z$ can be decomposed into blocks of types 1, 2, and 3 , and

- every type 2 block, except possibly the leftmost one, has the length contained in $\left[L, L^{\prime}\right)$ and yields expansion 6 ,

- the leftmost type 2 block has the length contained in $[0, L]$ and yields expansion $K>0$,

- all subsequences of the form $1 \ldots 13$, except possibly the rightmost one, yield expansion

$$
\gamma_{k_{j}} \ldots \gamma_{k_{1}} \gamma_{k_{0}}
$$

$k_{i}$ being the lengths of the corresponding blocks,

- the rightmost sequence of the form $1 \ldots 13$ yields expansion

$$
\begin{array}{ll}
\gamma_{k_{j}} \ldots \gamma_{k_{1}} \gamma_{k_{0}} \Delta^{\left(1-\mu(c) / \mu_{\max }\right)} & \text { if a critical point } c \in B_{\Delta}(z) \\
\gamma_{k_{j}} \ldots \gamma_{k_{1}} \gamma_{k_{0}} \Delta^{\left(1-1 / \mu_{\max }\right)} & \text { if otherwise. }
\end{array}
$$

\subsection{Inductive decomposition of backward orbits}

Let $z$ be a point which satisfies the assumptions of Lemma 3.2. We fix $N$ and a sequence of the preimages $F^{-N}(z), \ldots, F^{-1}(z), z$. We will split this sequence in the subsequences of the first, second, and third types.

Namely we will define by induction sequences $\left\{n_{j}\right\}$ and $\left\{z_{j}:=F^{-n_{j}}(z)\right\}$ such that $n_{0}=$ $0, n_{m-1}>N-L, n_{m}=N$, and

I) For every $j>0$ the sequence $F^{-n_{j}} z, \ldots, F^{-n_{j-1}} z$ is of the first, second, or third type.

II) For $j>0$ either the sequence $F^{-n_{j}} z, \ldots, F^{-n_{j-1}} z$ is of the second type (case IIa)), or some critical value $F\left(c_{j}\right) \in B_{R}\left(F z_{j}\right)$ (case IIb)).

Some additional properties will be discussed in the process of the construction. 
Base of induction. If the shrinking neighborhoods for $B_{2 R^{\prime}}\left(z_{0}\right)$ do not contain critical points, we set $n_{1}:=L, z_{1}:=F^{-L} z$, and the condition IIa) is satisfied for $z_{1}$. We start from $j=1$ and continue the inductive procedure as below.

By Lemma 3.1, dist $\left(z_{j}, J\right)<R^{\prime} / 2$, and hence sequences of the second type will yield desired expansion.

Otherwise we take $r:=\Delta$. By the choice of $\Delta$, the shrinking neighborhoods for $B_{\Delta}(z)$ omit the critical points. We increase $r$ continuously until certain shrinking neighborhood $U_{k}$ hits some critical point $c$, i.e. $c \in \partial U_{k}$. It must happen for some $r=r_{0}$ with $\Delta<r_{0}<2 R^{\prime}$. Set $n_{1}:=k$. Then $z_{1}$ is the third type preimage of $z_{0}$, and the condition IIb) for $z_{1}$ is satisfied by Lemma 2.3 .

Inductive procedure. Suppose we have already constructed $z_{j}$.

Case IIa. We enlarge the ball $B_{r}\left(z_{j}\right)$ continuously increasing the radius $r$ from 0 until one of the following conditions occurs:

1) for some $k$ the shrinking neighborhood $U_{k}$ for $B_{r}\left(z_{j}\right)$ hits some critical point $c^{\prime}, c^{\prime} \in \partial U_{k}$,

2) the radius $r$ reaches the value of $2 R^{\prime}$.

In the case 1$)$ we put $n_{j+1}:=n_{j}+k$. The condition I) is satisfied: $z_{j+1}$ is the third type preimage of $z_{j}$. The condition IIb) is satisfied by Lemma 2.5 .

In the case 2) set $n_{j+1}:=n_{j}+L$. Then $z_{j+1}$, $\operatorname{dist}\left(z_{j+1}, J_{F}\right)<R^{\prime} / 2$ is the second type preimage of $z_{j}$ of the length $L$. Clearly, $z_{j+1}$ satisfies conditions I) and IIa).

Case IIb. Suppose that we have IIb), but not IIa). Set $r=0$. The shrinking neighborhoods $U_{l}$ for $B_{r}\left(z_{j}\right), l \leq N-n_{j}$, do not contain critical points. We increase $r$ continuously until some domain $U_{k}$ hits a critical point $c^{\prime}, c^{\prime} \in \partial U_{k}$. This must occur for some $r<2 R^{\prime}$, since IIa) is not satisfied for $z_{j}$.

Let $n_{j+1}:=n_{j}+k$. Then the condition I) is satisfied: $z_{j+1}$ is the first type preimage of $z_{j}$. Lemma 2.3 implies the condition IIb).

Coding. As a result of the inductive procedure, we have decomposed the backward orbit of the point $z$ into pieces of type 1,2 and 3. This gives a coding of backward orbits by sequences of 1's, 2's and 3's which are always read from right to left. Not all combinations of the entries are admissible here. By the construction, type 3 is always preceded by type 2 except the coding sequence starts with 3 . For example we could have a sequence of the form

$$
\text { ...11111323222211113221113 , }
$$

$F$ acts from the left to the right and our inductive procedure has started from the right end. All pieces of the second type except maybe for the very last one have the length $L$.

This decomposition of backward orbits into pieces of different types is by no means the only one satisfying the desired properties. On the contrary, in the next Subsection we will have to reshuffle the coding slightly to obtain the claim of Lemma 3.2. 


\subsection{Estimates of expansion}

Growth of the derivative along sequences containing $1 \ldots 13$. Consider a sequence $1 \ldots 132$ obtained in the inductive construction. We will estimate expansion along a part of the orbit corresponding to its shorter sequence $1 \ldots 13$. Suppose that in the sequence $1 \ldots 132$ the consecutive pieces of type 1 have the lengths $k_{i}, i=1, \ldots, j$, and the piece of type 3 has the length $k_{0}$. Let $k=k_{0}+\ldots+k_{j}$. By Lemma 2.3 and Lemma 2.5 with $\mu:=\mu_{\max }$ we have that

$$
\begin{aligned}
\left|\left(F^{k}\right)^{\prime}(y)\right|^{\mu_{\max }}> & \prod_{i=1}^{j} \alpha_{k_{i}}\left(\gamma_{k_{i}}\right)^{\mu_{\max }} 2^{\mu_{\max }} \frac{d_{i+1}^{\mu_{i+1}-1}}{\left(r_{i+1}^{\prime}\right)^{\mu_{\max }-1}} \frac{r_{i}^{\mu_{\max }-1}}{\left(r_{i}+d_{i}\right)^{\mu_{i}-1}} \\
> & \left(C_{3 t} \alpha_{k_{1}}\left(\gamma_{k_{1}}\right)^{\mu_{\max }} \frac{\left(d_{2}\right)^{\mu_{2}-1}}{\left(r_{2}^{\prime}\right)^{\mu_{\max }-1}}\left(\prod_{i=0}^{j} \alpha_{k_{i}}\left(\gamma_{k_{i}}\right)^{\mu_{\max }-1}\right) \cdot\left(R_{2 t}\right)^{\mu_{\max }-1} \cdot \frac{d_{j+1}^{\mu_{j+1}-1}}{\left(r_{j+1}^{\prime}\right)^{\mu_{\max }-1}}\right. \\
& \left(\prod_{i=1}^{j} 2^{\mu_{\max }} \frac{d_{i}^{\mu_{i}-1}}{\left(r_{i}^{\prime}\right)^{\mu_{\max }-1}} \frac{r_{i}^{\mu_{\max }-1}}{\left(r_{i}+d_{i}\right)^{\mu_{i}-1}}\right) .
\end{aligned}
$$

Since $r_{i}^{\prime}<\min \left(r_{i}, d_{i}\right)$ and $\mu_{i} \leq \mu_{\max }$, we obtain that

$$
2^{\mu_{\max }} \frac{d_{i}^{\mu_{i}-1}}{\left(r_{i}^{\prime}\right)^{\mu_{\max }-1}} \frac{r_{i}^{\mu_{\max }-1}}{\left(r_{i}+d_{i}\right)^{\mu_{i}-1}}>1 .
$$

Also $r_{j+1}^{\prime}<d_{j+1}$ and

$$
\frac{d_{j+1}^{\mu_{j+1}-1}}{\left(r_{j+1}^{\prime}\right)^{\mu_{\max }-1}}>1 .
$$

Combining (11) and the estimates above, we obtain that

$$
\left|\left(F^{k}\right)^{\prime}(y)\right|^{\mu_{\max }}>\left(R_{2 t}\right)^{\mu_{\max }-1} \cdot C_{3 t} \prod_{i=0}^{j} \alpha_{k_{j}}\left(\gamma_{k_{i}}\right)^{\mu_{\max }} .
$$

If the rightmost sequence $1 \ldots 13$ is not preceded by 2 then similarly as above, using Lemma 2.3, we obtain that

$$
\left|\left(F^{k}\right)^{\prime}(y)\right|^{\mu_{\max }}>C_{3 t} \prod_{i=0}^{j} \alpha_{k_{j}}\left(\gamma_{k_{i}}\right)^{\mu_{\max }} \cdot \Delta^{\mu_{\max }-\mu(c)}
$$

if there is a critical point $c$ inside $B_{\Delta}(z)$. Otherwise we use Lemma 2.5 instead to infer that

$$
\left|\left(F^{k}\right)^{\prime}(y)\right|^{\mu_{\max }}>C_{3 t} \prod_{i=0}^{j} \alpha_{k_{j}}\left(\gamma_{k_{i}}\right)^{\mu_{\max }} \cdot \Delta^{\mu_{\max }-1} .
$$


Derivation of the Main Lemma. Consider a sequence of the preimages $F^{-m}(z), \ldots, z$ with a coding sequence of the form

$$
\text { .. } 11111323222211113221113 \text {. }
$$

If the lenght $k_{1}+\ldots+k_{j}$ of a piece of the backward orbit of the form $11 \ldots 13$ is large enough then the expansion prevails over accumulation of distortion and small scale constants in the estimate (13). Otherwise $k_{1}+\ldots+k_{j}$ is uniformly bounded and the whole piece of the backward orbit will be treated as type 2 . Consequently, we will convert the code $1 \ldots 132$ into 2 .

We proceed with estimates along the above lines to complete the proof of Lemma 3.2. Since $\lim _{i \rightarrow \infty} \alpha_{i}=\infty$, there exists $\tau$ such that $\alpha_{i} \geq 8$ for $i \geq \tau$. Set

$$
\alpha_{n}^{\prime}:=\inf \left\{\prod_{j} \alpha_{i_{j}}: i_{0}+i_{1}+i_{2}+\ldots \geq n ; i_{1}, i_{2}, \ldots \geq \tau\right\},
$$

and observe that $\lim _{n \rightarrow \infty} \alpha_{n}^{\prime}=\infty$. Now we choose large $L^{\prime \prime}$ so that for $n \geq L^{\prime \prime}$ one has

$$
\alpha_{n}^{\prime} C_{3 t}\left(R_{2 t}\right)^{\mu_{\max }-1} \geq 1 \text {. }
$$

A new coding of the preimages $F^{-m}(z), \ldots, z$ is designed as follows: take a piece of the backward orbit corresponding to a subsequence $1 \ldots 132$ of the length $k$. The consecutive pieces (counted from the right to the left) have the lengths $k_{i}, i=0, \ldots, j$. Consider two possible cases:

1) If $k<L^{\prime \prime}$ then $1 \ldots 132$ is replaced by 2 . The corresponding block of the preimages is indeed of type 2 and the length $n:=L+k$ with $n<L^{\prime}:=\left(L+L^{\prime \prime}\right)$.

2) If $k \geq L^{\prime \prime}$ then $1 \ldots 132$ remains unchanged and by the estimate (13) and the definition of $L^{\prime \prime}$, the derivative of $F^{k}(y)$ is greater than $\gamma_{k_{j}} \cdots \gamma_{k_{1}}$. The last pair of estimates of the Main Lemma 3.2 follows immediately from (14) and (15) and the definition of $L^{\prime \prime}$.

The proof of the Main Lemma 3.2 is completed.

Strong expansion along some sequences $11 \ldots 1$. Consider a subsequence $11 \ldots 1$ obtained in the inductive construction. Suppose that $x$ is a terminal point of this subsequence and the consecutive pieces of type 1 have lengths $k_{i}, i=0, \ldots, j$. Following the notation of Lemma 3.2, we prove the lemma below, which will be later used in our investigation of conformal and invariant densities.

Lemma 3.3 Let $G$ be a set of indexes $j$ such that $d_{j}<r_{j}$ and $\mu_{\text {max }}^{\prime}$ be the maximal multiplicity which occurs in the sequence $\left\{\mu_{j}: j \in G\right\}$. Choose an index $j$ from the set $G^{\prime}:=$ $\left\{j \in G: \mu_{j}=\mu_{\max }^{\prime}\right\}$ and denote $k=\sum_{i=0}^{j-1} k_{i}$. Then

$$
\left|\left(F^{k}\right)^{\prime}(x)\right|>\prod_{i=0}^{j-1} \gamma_{k_{i}} \text {. }
$$


Proof: If $\mu:=\mu_{\max }^{\prime}$ then Lemma 2.3 and Lemma 2.5 imply a counterpart of the estimate (11),

$$
\begin{aligned}
\left|\left(F^{k}\right)^{\prime}(x)\right|^{\mu_{\max }^{\prime}}> & \prod_{i=0}^{j-1} \alpha_{k_{i}}\left(\gamma_{k_{i}}\right)^{\mu_{\max }^{\prime}} 2^{\mu_{\max }^{\prime}} \frac{d_{i+1}^{\mu_{i+1}-1}}{\left(r_{i+1}^{\prime}\right)^{\mu_{\text {max }}^{\prime}-1}} \frac{r_{i}^{\mu_{\text {max }}^{\prime}-1}}{\left(r_{i}+d_{i}\right)^{\mu_{i}-1}} \\
> & \left(\prod_{i=0}^{j-1} \alpha_{k_{i}}\left(\gamma_{k_{i}}\right)^{\mu_{\max }^{\prime}}\right) \cdot \frac{d_{0}^{\mu_{0}-1}}{\left(r_{0}^{\prime}\right)^{\mu_{\max }^{\prime}-1}} \\
& \cdot\left(\prod_{i=0}^{j-1} 2^{\mu_{\max }^{\prime}} \frac{d_{i}^{\mu_{i}-1}}{\left(r_{i}^{\prime}\right)^{\mu_{\max }^{\prime}-1}} \frac{r_{i}^{\mu_{\text {max }}^{\prime}-1}}{\left(r_{i}+d_{i}\right)^{\mu_{i}-1}}\right) \cdot \frac{r_{j}^{\mu_{\text {max }}^{\prime}-1}}{\left(r_{j}+d_{j}\right)^{\mu_{j}-1}} .
\end{aligned}
$$

We cannot proceed exactly as in (11), since it was essential that $\mu_{\max }$ was the maximal multiplicity. Instead, we use the properties of $\mu_{\max }^{\prime}$ and the set $G$ as follows:

(i) If $i \in G$ then $r_{i}^{\prime}<\min \left(r_{i}, d_{i}\right)$ and $\mu_{i} \leq \mu_{\max }^{\prime}$. We see that the estimate (12) holds with $\mu_{\max }$ replaced by $\mu_{\max }^{\prime}$,

$$
2^{\mu_{\text {max }}^{\prime}} \frac{d_{i}^{\mu_{i}-1}}{\left(r_{i}^{\prime}\right)^{\mu_{\text {max }}^{\prime}-1}} \frac{r_{i}^{\mu_{\text {max }}^{\prime}-1}}{\left(r_{i}+d_{i}\right)^{\mu_{i}-1}}>1 .
$$

(ii) If $i \notin G$ then $d_{i} \geq r_{i}>r_{i}^{\prime}$ and the same estimate is still valid,

$$
2^{\mu_{\text {max }}^{\prime}} \frac{d_{i}^{\mu_{i}-1}}{\left(r_{i}^{\prime}\right)^{\mu_{\text {max }}^{\prime}-1}} \frac{r_{i}^{\mu_{\text {max }}^{\prime}-1}}{\left(r_{i}+d_{i}\right)^{\mu_{i}-1}} \geq 2^{\mu_{\max }} \frac{d_{i}^{\mu_{i}-1}}{\left(2 d_{i}\right)^{\mu_{i}-1}} \frac{r_{i}^{\mu_{\max }^{\prime}-1}}{\left(r_{i}^{\prime}\right)^{\mu_{\max }^{\prime}-1}}>1 .
$$

(iii) By our choice, $j \in G^{\prime}$. This means that $d_{j}<r_{j}$ and $\mu_{j}=\mu_{\text {max }}^{\prime}$. Hence,

$$
2^{\mu_{\text {max }}^{\prime}} \frac{r_{j}^{\mu_{\text {max }}^{\prime}-1}}{\left(r_{j}+d_{j}\right)^{\mu_{j}-1}} \geq 2^{\mu_{\max }} \frac{r_{j}^{\mu_{\max }^{\prime}-1}}{\left(2 r_{j}\right)^{\mu_{\max }^{\prime}-1}}>1
$$

(iv) By the definition, $r_{0}^{\prime}<d_{0}$ and

$$
\frac{d_{0}^{\mu_{0}-1}}{\left(r_{0}^{\prime}\right)^{\mu_{\max }^{\prime}-1}}>1
$$

Inserting the estimates (i) - (iv) into (16) we obtain the claim of the lemma.

Proof of Proposition 2.1. Fix a point $z$ sufficiently close to the Julia set and a branch of $F^{-n}$ at $z$. By Lemma 2.2,

$$
\sum_{n}\left(\gamma_{n}\right)^{-\beta}<1 / 4, \quad \beta:=\frac{\mu_{\max } \alpha}{1-\alpha} .
$$


Let $\gamma_{n}^{\prime}:=\inf \left\{\prod_{j} \gamma_{i_{j}}: i_{0}+i_{1}+i_{2}+\ldots=n\right\}$. By simple algebra,

$$
\begin{aligned}
\sum_{n}\left(\gamma_{n}^{\prime}\right)^{-\beta} & <\sum_{n}\left(\gamma_{n}\right)^{-\beta}+\left(\sum_{n}\left(\gamma_{n}\right)^{-\beta}\right)^{2}+\left(\sum_{n}\left(\gamma_{n}\right)^{-\beta}\right)^{3}+\ldots \\
& <\frac{1}{4}+\left(\frac{1}{4}\right)^{2}+\left(\frac{1}{4}\right)^{3}+\ldots=\frac{1}{3}
\end{aligned}
$$

Lemma 3.2 gives a decomposition of the orbit $F^{-n}(z), \ldots, F^{-1}(z), z$ into pieces of type 1 , 2 , and 3 , with the following properties (we restate them using new notation):

(i) each piece of the form $1 \ldots 13$ of length $k$, except the rightmost one, yields expansion $\gamma_{k}^{\prime}$,

(ii) the rightmost piece of the form $1 \ldots 13$ of length $k$ yields expansion $\gamma_{k}^{\prime} \Delta^{1 / \mu_{\max }-1}$,

(iii) each piece of the form 2, except possibly the leftmost one, has the length $l \in\left[L, L^{\prime}\right.$ ) and yields expansion $6 \geq \lambda^{l}$, where $\lambda:=6^{1 / L^{\prime}}>1$,

(iv) the leftmost piece of the form 2 , has the length $l \in[0, L)$ and yields expansion $K$.

If we set

$$
\omega_{n}:=\inf \left\{K \lambda^{k_{0}} \prod_{j \geq 1} \gamma_{k_{j}}^{\prime}: k_{0}+k_{1}+k_{2}+\ldots \in[n-L, n)\right\},
$$

then properties (i)-(iv) above clearly imply

$$
\left|\left(F^{n}\right)^{\prime}\left(F^{-n} z\right)\right|>\Delta^{1-1 / \mu_{\max }} \omega_{n} .
$$

On the other hand,

$$
\begin{aligned}
\sum_{n}\left(\omega_{n}\right)^{-\beta}< & K^{-\beta}\left(1+\lambda^{-\beta}+\lambda^{-2 \beta}+\ldots\right) \\
& \cdot\left(\sum_{n}\left(\gamma_{n}^{\prime}\right)^{-\beta}+\left(\sum_{n}\left(\gamma_{n}^{\prime}\right)^{-\beta}\right)^{2}+\left(\sum_{n}\left(\gamma_{n}^{\prime}\right)^{-\beta}\right)^{3}+\ldots\right) \\
< & K^{-\beta}\left(1-\lambda^{-\beta}\right)^{-1}\left(\frac{1}{3}+\left(\frac{1}{3}\right)^{2}+\left(\frac{1}{3}\right)^{3}+\ldots\right) \\
< & K^{\beta}\left(1-\lambda^{-\beta}\right)^{-1} \frac{1}{2}<\infty
\end{aligned}
$$

which completes the proof of the first inequality of Proposition 2.1. The proof of the second, when a critical point $c \in B_{\Delta}(z)$, is very much the same. 


\subsection{Summability along backward orbits}

Fix a point $z$ and a positive number $\Delta$. Let $\mathcal{H}(z, \Delta)$ stand for the set of all preimages of $z$ such that a ball $B_{\Delta}(z)$ can be pulled back univalently along the corresponding branch. By Lemma 3.2, every backward orbit of $z$ which terminates at $y \in \mathcal{H}(z, \Delta)$ can be decomposed into blocks of type 1,2 , or 3 .

Definition 3.2 In the decomposition of the Main Lemma, let $x \in \mathcal{H}(z, \Delta)$ be a point which starts a type 3 block. Denote by $\mathbf{I}(x \mid z)=\mathbf{I}^{\Delta}(x \mid z)$ the set of all $y \in \mathcal{H}(z, \Delta)$ which are the endpoints of type 1 blocks preceded by exactly one type 3 block beginning at $x$. For example, preimages of $x$ which are the endpoints of blocks $13,113, \ldots$ belong to $\mathbf{I}(x \mid z)$.

Let $L^{\prime}>L$ be the constants supplied by the Main Lemma. In the decomposition of the Main Lemma, let $x \in \mathcal{H}(z, \Delta)$ be a point which starts a type 2 block. Denote by $\mathbf{I I}_{l}(x \mid z)$ and $\mathbf{I I}_{s}(x \mid z)$, respectively, the sets of all "long" (of order $L^{\prime}>n(y) \geq L$ ) and "short" (of order $n(y)<L$ ) type 2 preimages $y$ of $x$.

Note that the definition of $\mathbf{I}(x \mid z)=\mathbf{I}^{\Delta}(x \mid z)$ depends on the choice of $\Delta$. Also, the definitions of $\mathbf{I I}_{l}(x \mid z)$ and $\mathbf{I I}_{s}(x \mid z)$ depend on the choice of $\Delta$, however all estimates from Lemma 2.4 are independent of $\Delta$, so we simplify the notation by omitting $\Delta$.

Note also that $z$ is its own preimage of order zero (since formally $F^{0}(z)=z$ ), so we write, e.g. $y \in \mathbf{I}(z \mid z)$ if $y \in \mathcal{H}(z, \Delta)$ is the endpoint of a type 1 block preceded by exactly one type 3 block beginning at $z$.

We will drop $z$ from the notation of $\mathbf{I}(x \mid z), \mathbf{I I}_{s}(x \mid z), \mathbf{I I}_{l}(x \mid z)$ whenever no confusion can arise.

Lemma 3.4 Let $\beta=\mu_{\max } \alpha /(1-\alpha)$. If a rational function $F$ satisfies the summability condition with an exponent $\alpha \leq 1$ then there exists $\epsilon>0$ so that for every point $z$ from $\epsilon$-neighborhood of the Julia set $J$ and every set $\mathbf{I}(x \mid z)=\mathbf{I}^{\Delta}(x \mid z)$,

$$
\begin{array}{ll}
\sum_{y \in \mathbf{I}(x \mid z)}\left|\left(F^{n(y)}\right)^{\prime}(y)\right|^{-\beta}<\frac{1}{3} & \text { if } x \neq z, \\
\sum_{y \in \mathbf{I}(x \mid z)}\left|\left(F^{n(y)}\right)^{\prime}(y)\right|^{-\beta}<\frac{1}{3} \Delta^{\beta\left(1 / \mu_{\max }-1\right)} & \text { if } x=z, \\
\sum_{y \in \mathbf{I}(x \mid z)}\left|\left(F^{n(y)}\right)^{\prime}(y)\right|^{-\beta}<\frac{1}{3} \Delta^{\beta\left(\mu(c) / \mu_{\max }-1\right)} & \text { if } x=z \text { and a critical point } c \in B_{\Delta}(z) .
\end{array}
$$

Proof: We will work with sequences $\alpha_{n}, \gamma_{n}$, and $\delta_{n}$ supplied by Lemma 2.2. Recall that $\sum_{n}\left(\gamma_{n}\right)^{-\beta}<1 /(16 \operatorname{deg} F)$.

Observe that any point $y \in F^{-k}(z)$ has at most $4 \operatorname{deg} F$ preimages of a given length which are of the first or the third type. In fact, since pull-backs to the critical values are univalent, there is only one way to hit a specific critical value after particular number of steps, and thus only $\mu(c)$ ways to hit a critical point $c$, but

$$
\sum_{c} \mu(c)=\#\{\operatorname{Crit}\}+\sum_{c}(\mu(c)-1) \leq 2(\operatorname{deg} F-1)+2(\operatorname{deg} F-1)<4 \operatorname{deg} F .
$$


Therefore, for every sequence $k_{0}, k_{1}, \ldots, k_{m}$ of positive integers there are at most $(2 \mathrm{deg} F)^{m+1}$ sequences $1 \ldots 13$ with the corresponding lengths of the pieces of type 1 and 3 . By the Main Lemma 3.2, if $x \neq z$ then for every $y \in \mathbf{I}(x)$

$$
\left|\left(F^{n(y)}\right)^{\prime}(y)\right| \geq \gamma_{k_{0}} \gamma_{k_{1}} \ldots \gamma_{k_{m}}
$$

and

$$
\begin{aligned}
\sum_{y \in \mathbf{I}(x)}\left|\left(F^{n(y)}\right)^{\prime}(y)\right|^{-\beta} & <\sum_{m, k_{0}, k_{1}, \ldots, k_{m}}(4 \operatorname{deg} F)^{m+1}\left(\gamma_{k_{0}} \gamma_{k_{1}} \ldots \gamma_{k_{m}}\right)^{-\beta} \\
& <4 \operatorname{deg} F \sum_{k} \gamma_{k}^{-\beta}+\left(4 \operatorname{deg} F \sum_{k} \gamma_{k}^{-\beta}\right)^{2}+\left(4 \operatorname{deg} F \sum_{k} \gamma_{k}^{-\beta}\right)^{3}+\ldots \\
& <\frac{1}{4}+\left(\frac{1}{4}\right)^{2}+\left(\frac{1}{4}\right)^{3}+\ldots=\frac{1}{3} .
\end{aligned}
$$

If $x=z$ then the rightmost sequence begins with 3 . Similarly as before, using the estimates of the Main Lemma, we obtain that

$$
\begin{aligned}
& \sum_{y \in \mathbf{I}(z)}\left|\left(F^{n(y)}\right)^{\prime}(y)\right|^{-\beta}<\frac{1}{3} \Delta^{\beta\left(1 / \mu_{\max }-1\right)}, \quad \text { or } \\
& \sum_{y \in \mathbf{I}(z)}\left|\left(F^{n(y)}\right)^{\prime}(y)\right|^{-\beta}<\frac{1}{3} \Delta^{\beta\left(\mu(c) / \mu_{\max }-1\right)}, \quad \text { if a critical point } c \in B_{\Delta}(z) .
\end{aligned}
$$

This completes the proof of Lemma 3.4.

Lemma 3.5 Assume that the Poincaré series with exponent $q$ is summable for some point $v \in \hat{\mathbb{C}}$. Then there exists $\epsilon>0$ so that for every point $z$ from $\epsilon$-neighborhood of the Julia set $J$ and every set $\mathbf{I I}_{l}(x \mid z)$ and $\mathbf{I I}_{s}(x \mid z)$,

$$
\begin{aligned}
& \sum_{y \in \mathbf{I I}_{l}(x \mid z)}\left|\left(F^{n(y)}\right)^{\prime}(y)\right|^{-q}<\frac{1}{36}, \\
& \sum_{y \in \mathbf{I I}_{s}(x \mid z)}\left|\left(F^{n(y)}\right)^{\prime}(y)\right|^{-p}<C(p) \text { for any } p .
\end{aligned}
$$

Proof: This is a reformulation of Lemma 2.4 in the new notation.

\section{Poincaré series}

In this Section we analyze Poincaré series, particularly proving a self-improving property of the Poincaré exponent. Theorem 1 is a direct consequence of this property. We recall that $\mathcal{H}(z, \Delta)$ stands for the set of all preimages $F^{-n} z, n \in \mathbb{N}$, such that the ball $B_{\Delta}(z)$ can be pulled back univalently along the corresponding branch of $F^{-n}$. 
Proposition 4.1 (Self-improving property of the Poincaré exponent) Suppose that a rational function $F$ satisfies the summability condition with an exponent

$$
\alpha<\frac{q}{\mu_{\max }+q},
$$

$q>0$, and the Poincaré series with exponent $q$ converges for some point $v, \Sigma_{q}(v)<\infty$. Then there exist $p<q, \epsilon>0$, and $C(\epsilon, p)$ so that for every point $z$ in the $\epsilon$-neighborhood of the Julia set

$$
\begin{aligned}
& \sum_{y \in \mathcal{H}(z, \Delta)}\left|\left(F^{n}\right)^{\prime}(y)\right|^{-p}<C \Delta^{p\left(\frac{\mu(c)}{\mu_{\max }}-1\right)} \quad \text { if a critical point } c \in B_{\Delta}(z), \\
& \sum_{y \in \mathcal{H}(z, \Delta)}\left|\left(F^{n}\right)^{\prime}(y)\right|^{-p}<C \Delta^{p\left(\frac{1}{\mu_{\max }}-1\right)} \quad \text { otherwise. }
\end{aligned}
$$

Corollary 4.1 Assume that $F$ satisfies the summability condition with an exponent

$$
\alpha<\frac{q}{\mu_{\max }+q},
$$

$q>0$, and there exists a point $z \in \hat{\mathbb{C}}$ so that the Poincaré series $\Sigma_{q}(z)$ converges. Then

- $\delta_{\text {Poin }}(w)<q$ if $w$ is at a positive distance from the orbits of the critical points,

- $\delta_{\text {Poin }}(c)<q$ if $c$ is a critical point of the maximal multiplicity.

Proof: If the distance of $w$ to the critical orbits in $J$ is positive then all preimages of $w$ belong to $\mathcal{H}(w, \Delta)$ with $\Delta$ sufficiently small. This yields $\Sigma_{p}(w)<\infty$.

If $c$ is a critical point of the maximal multiplicity $\mu(c)=\mu_{\max }$ then

$$
\sum_{y \in \mathcal{H}(c, \Delta)}\left|\left(F^{n}\right)^{\prime}(y)\right|^{-p}<C \Delta^{p\left(\frac{\mu(c)}{\mu_{\max }}-1\right)}=C,
$$

and letting $\Delta$ go to zero we obtain that

$$
\sum_{n} \sum_{y \in F^{-n} c}\left|\left(F^{n}\right)^{\prime}(y)\right|^{-p}<C
$$

Corollary 4.2 If a rational function $F$ satisfies the summability condition with an exponent $\alpha<2 /\left(\mu_{\max }+2\right)$ and its Julia set is not the whole sphere, then there exists $p<2$ so that the conclusion of Proposition 4.1 holds.

Proof: If the Julia set is not the whole sphere and the Fatou set does not contain elliptic components then there exists a point $v \in \hat{\mathbb{C}} \backslash J$ such that the Poincaré series $\Sigma_{2}(v)$ converges. This a classical area argument, [45]. It is enough to notice that there exists a small ball $B_{\delta}(v)$ free from the critical orbits and with preimages pairwise disjoint. 
Proof of Theorem 1. Suppose that the Poincaré series $\Sigma_{q}(v)$ converges for a point $v \in \hat{\mathbb{C}}$. If $q \leq \delta_{\text {Poin }}(J)$ then, by Corollary 4.1 , there exist $\epsilon>0$ so that $\delta_{\text {Poin }}(J) \leq q-\epsilon<\delta_{\text {Poin }}(J)$, a contradiction. This means that for $q=\delta_{P o i n}(J)$, the Poincaré series $\Sigma_{q}(z)$ diverges for every point $z \in \hat{\mathbb{C}}$. Hence, $\delta_{\text {Poin }}(z) \geq \delta_{\text {Poin }}(J)$ for every $z \in \hat{\mathbb{C}}$.

By the definition of the Poincaré exponent $\delta_{\text {Poin }}(J)$, for any $\epsilon>0$ there exist $q<\delta_{\text {Poin }}(J)+\epsilon$ and a point $v \in \hat{\mathbb{C}}$ so that the Poincaré series $\Sigma_{q}(v)$ converges. By Corollary 4.1, for all points which are at the positive distance to the critical orbits and for all critical points of maximal multiplicity one has $\delta_{\text {Poin }}(z)<\delta_{\text {Poin }}(J)+\epsilon$ and Theorem 1 follows.

Proof of Proposition 4.1. We use the inductive decomposition of backward orbits described in Section 3.1. Let $z$ be a point from an $\epsilon$-neighborhood of the Julia set. By Lemma 3.5,

$$
\sum_{y \in \mathbf{I I}_{l}(x)}\left|\left(F^{n(y)}\right)^{\prime}(y)\right|^{-q}<\frac{1}{36} .
$$

But there are at most $(\operatorname{deg} F)^{L^{\prime}}$ points in $\mathbf{I I}_{l}(x)$, since these preimages are all of the order at most $\left(L^{\prime}-1\right)$. Therefore by power means inequality (see e.g. Section 2.9 in [21]) we have for $p<q$ sufficiently close to $q$ (namely $\left.p>q-q \log 2\left(L^{\prime} \log (\operatorname{deg} F)\right)^{-1}\right)$ :

$$
\begin{aligned}
\sum_{y \in \mathbf{I I}_{l}(x)}\left|\left(F^{n(y)}\right)^{\prime}(y)\right|^{-p} & <\left(\sum_{y \in \mathbf{I I}_{l}(x)}\left|\left(F^{n(y)}\right)^{\prime}(y)\right|^{-q}\right)^{\frac{p}{q}} \cdot(\operatorname{deg} F)^{L^{\prime} \frac{q-p}{q}} \\
& <\left(\frac{1}{36}\right)^{\frac{p}{q}} \cdot(\operatorname{deg} F)^{L^{\prime} \frac{q-p}{q}} \\
& <\frac{1}{6} \cdot(\operatorname{deg} F)^{L^{\prime} \frac{q-p}{q}}<\frac{1}{3} .
\end{aligned}
$$

Also by Lemma 3.5

$$
\sum_{y \in \mathbf{I} \mathbf{I}_{s}(x)}\left|\left(F^{n(y)}\right)^{\prime}(y)\right|^{-p}<C=C(p) .
$$

We expand $\sum_{y \in \mathcal{H}(z, \Delta)}\left|\left(F^{n}\right)^{\prime}(y)\right|^{-p}$ by grouping preimages of the same kind into clusters. We begin with $z$ obtaining preimages of three kinds: $\mathbf{I}(z)=\mathbf{I}^{\Delta}(z), \mathbf{I I}_{l}(z)$ and $\mathbf{I I}_{s}(z)$. Points in $\mathbf{I I}_{s}(z)$ are terminal while preimages $y$ of the points in $\mathbf{I}(z)$ and $\mathbf{I I}_{l}(z)$ are divided further. We proceed in this fashion down the tree of preimages of $z$. If there is no critical point in $B_{\Delta}$ we obtain that

$$
\begin{aligned}
\sum_{y \in \mathcal{H}(z, \Delta)}\left|\left(F^{n}\right)^{\prime}(y)\right|^{-p}= & \sum_{z^{\prime} \in \mathbf{I I}_{s}(z)} \mid\left(F^{\left.n\left(z^{\prime}\right)\right)\left.^{\prime}\left(z^{\prime}\right)\right|^{-p}+} \sum_{z^{\prime} \in \mathbf{I}, \mathbf{I} \mathbf{I}_{l}(z)}\left|\left(F^{n\left(z^{\prime}\right)}\right)^{\prime}\left(z^{\prime}\right)\right|^{-p}\right. \\
& \cdot\left(\sum_{z^{\prime \prime} \in \mathbf{I I}_{s}\left(z^{\prime}\right)}\left|\left(F^{n\left(z^{\prime \prime}\right)}\right)^{\prime}\left(z^{\prime \prime}\right)\right|^{-p}+\sum_{z^{\prime \prime} \in \mathbf{I}_{,}, \mathbf{I}_{l}\left(z^{\prime}\right)}\left|\left(F^{n\left(z^{\prime \prime}\right)}\right)^{\prime}\left(z^{\prime \prime}\right)\right|^{-p}\right.
\end{aligned}
$$




$$
\begin{aligned}
& \left.\left(\sum_{z^{\prime \prime \prime} \in \mathbf{I I}_{s}\left(z^{\prime \prime}\right)}\left|\left(F^{n\left(z^{\prime \prime \prime}\right)}\right)^{\prime}\left(z^{\prime \prime \prime}\right)\right|^{-p}+\ldots\right)\right) \\
\leq & C+\left(\frac{1}{3}+\frac{1}{3}(\Delta)^{p\left(1 / \mu_{\max }-1\right)}\right)\left(C+\frac{2}{3}(C+\ldots)\right) \\
= & C+\frac{1}{3}\left(1+(\Delta)^{p\left(1 / \mu_{\max }-1\right)}\right) C\left(1+\frac{2}{3}+\left(\frac{2}{3}\right)^{2}+\ldots\right) \\
= & \left(2+(\Delta)^{p\left(1 / \mu_{\max }-1\right)}\right) C<3 C(\Delta)^{p\left(1 / \mu_{\max }-1\right)} .
\end{aligned}
$$

Otherwise, we have a stronger estimate

$$
\sum_{y \in \mathcal{H}(z, \Delta)}\left|\left(F^{n}\right)^{\prime}(y)\right|^{-p}<3 C(\Delta)^{p\left(\mu(c) / \mu_{\max }-1\right)} .
$$

This proves Proposition 4.1.

\section{$5 \quad$ Induced hyperbolicity and conformal measures}

\subsection{Inductive procedure with a stopping rule}

We decompose a sequence of preimages $F^{-N}(z), \ldots, F^{-1}(z), z$ into blocks of types 2 and $1 \ldots 13$ using the usual inductive procedure with the following new stopping rule: at the first occurrence of a type 2 sequence we stop the induction. For the reader's convenience we will describe the construction.

Construction. We take shrinking neighborhoods $\left\{U_{k}\right\}$ for $B_{2 R^{\prime}}(z)$. If they do not contain the critical points we form one block of type 2 of the length $N$. Otherwise, we set $r=0$ and increase it continuously until some shrinking neighborhood $U_{k}$ hits a critical point $c, c \in \partial U_{k}$. It must happen for some $0<r<2 R^{\prime}$. Set $r_{0}:=\operatorname{dist}\left(F^{k}(c), z\right), n_{1}:=k$, and $z_{1}:=F^{-n_{1}}(z)$. Then $z_{1}$ is a third type preimage of $z$ and the ball $B_{r_{0}}$ can be pulled back univalently by $F^{N}$ along the backward orbit.

Inductive procedure. Suppose we have already constructed $z_{j}=F^{-n_{j}}(z)$ which is of type 1 or 3 . We enlarge the ball $B_{r}\left(z_{j}\right)$ continuously increasing the radius $r$ from 0 until one of the following conditions is met:

1) for some $k \leq N-n_{j}$ the shrinking neighborhood $U_{k}$ for $B_{r}\left(z_{j}\right)$ hits a critical point $c \in$ Crit, $c \in \partial U_{k}$,

2) radius $r$ reaches the value of $2 R^{\prime}$.

In the case 1$)$ we put $n_{j+1}:=n_{j}+k$. Clearly, $z_{j+1}:=F^{-n_{j+1}}(z)$ is a type 1 preimage of $z_{j}$. If 2) holds, we set $z_{j+1}:=F^{-N}(z)$ which is a type 2 preimage of $z_{j}$. This terminates the construction in this case. 
Coding. As a result of the inductive procedure, we can decompose the backward orbit of a point $z$ into pieces of type 1,2 and 3. This gives a coding of backward orbits by sequences of 1's, 2's and 3's. By the construction, only the following three types of codings are allowable: $2,1 \ldots 3,21 \ldots 13$. We recall that according to our convention, during the inductive procedure we put symbols in the coding from the right to the left.

We attach to every sequence of preimages of $z$ the sequence $k_{l}, \ldots, k_{0}$ of the lengths of the blocks of preimages of a given type in its coding. Again our convention requires that $k_{0}$ stands always for the length of the rightmost block of preimages in the coding. Clearly, $k_{0}+\cdots+k_{l}=N$.

\subsection{Most points go to large scale infinitely often}

We recall that a Jacobian of a $\delta$-conformal measure $\nu$ is equal to $\left|F^{\prime}\right|^{\delta}$ (see Definition 1.3),

$$
d \nu(F(z))=\left|F^{\prime}(z)\right|^{\delta} d \nu(z) .
$$

Consider a subset of points in $J$ which infinitely often go to the large scale of size $R^{\prime}$ with bounded distortion:

$$
J_{*}:=\left\{z \in J: \exists n_{j} \rightarrow \infty \text {, with } F^{n_{j}} \text { univalent on } F^{-n_{j}}\left(B\left(F^{n_{j}} z, R^{\prime}\right)\right)\right\} .
$$

Note that the value of $R^{\prime}$ is already fixed and does not depend on the point.

Proposition 5.1 Suppose that a rational function F satisfies the summability condition with an exponent

$$
\alpha<\frac{p}{\mu_{\max }+p} .
$$

Then for any p-conformal measure $\nu$ with no atoms at critical points $\nu\left(J \backslash J_{*}\right)=0$.

Proof: For every $x \in J$ and every $k \in \mathbb{N}$, we use the inductive procedure of Section 5.1 to decompose the sequence $x, \ldots, F^{k}(x)$ of preimages of $F^{k}(x)$ into blocks of either $211 \ldots 113$ or 2. The procedure is stopped at the first occurrence of type 2 block, which might be of arbitrary length. In particular, it might be of length zero which means that a block of type 2 does not occur and the sequence ends with type 1.

Denote by $\mathcal{E}_{x}$ the set of all codes obtained for $x$. Points in $J_{*}$ are precisely those for which we get infinitely many different type 2 sequences. Hence, if $x \in J \backslash J_{*}$, then $x$ is a terminal point of an infinite number of sequences $2111 \ldots 113$ with only a finite choice of type 2 blocks. Let $k(x)$ be the minimal number for which infinitely many sequences from $\mathcal{E}_{x}$ have the same type 2 block of length $k(x)$. Denote $X_{k}:=\{x: k(x)=k\}$ and observe that the sets $\left\{X_{k}: k=0,1, \ldots\right\}$ form a countable partition of $J \backslash J_{*}$.

If $\nu$ has no atoms at critical points and $F^{k}(X)$ is measurable then

$$
\nu\left(F^{k} X\right)=0 \Longleftrightarrow \nu(X)=0 \Longleftrightarrow \nu\left(F^{-k} X\right)=0 .
$$

Since $F^{k}\left(X_{k}\right) \subset X_{0}$ and consequently $J \backslash J_{*} \subset \cup_{k} F^{-k}\left(X_{0}\right)$, it is sufficient to prove that $\nu\left(X_{0}\right)=0$. Without loss of generality we can exclude from $X_{0}$ all preimages of the critical 
points since they are of zero $\nu$ measure. Every point $x \in X_{0}$ must be terminal for infinitely many different subsequences $1 \ldots 1$. Otherwise the orbit of $x \in X_{0}$ would pass near the critical points only finitely many times and hence its distance to the set Crit would be positive. Another consequence of the finitness of $1 \ldots 1$ subsequences would be an unbounded length of type 3 blocks in $\mathcal{E}_{x}$. The estimate (10) yields dist $(x$, Crit) $=0$, a contradiction.

By very much the same argument, using that the distance from $x \in X_{0}$ to Crit is positive, we obtain that the length of the leftmost blocks of type 1 in $\mathcal{E}_{x}$ must be bounded and therefore we can choose infinitely many sequences from $\mathcal{E}_{x}$ with the same leftmost block. Next, we consider the second block of type 1 from the left and repeat the above argument to produce infinitely many sequences in $\mathcal{E}_{x}$ with the same two leftmost blocks. We continue in this fashion until we build by induction an infinite sequence $1111 \ldots$ terminating at $x$. Denote corresponding parameters by $d_{j}, r_{j}, r_{j}^{\prime}, c_{j}, \mu_{j}, n_{j}$ with $j=0,-1,-2, \ldots$ (we use negative integers to preserve convention of enumerating from the right to the left).

Let $G$ be the set of indexes $j$ such that $d_{j}<r_{j}$. The second inequality of Lemma 2.3 implies that if $j \notin G$ then $\left(d_{j+1}\right)^{\mu_{j+1}}<\left(d_{j}\right)^{\mu_{j}}\left(\gamma_{n_{j}}\right)^{-\mu_{\max }}$. This means that $G$ is infinite since otherwise $\lim _{j \rightarrow-\infty} d_{j}=\infty$. Now set $\mu_{\text {max }}^{\prime}$ to be the maximal multiplicity which occurs infinitely often in the sequence $\left\{\mu_{j}: j \in G\right\}$. Let $X_{0}^{\prime}(k)$ stand for the set of all points $x \in X_{0}$ such that there are no points of larger than $k=\mu_{\text {max }}^{\prime}(x)$ multiplicity in $G$. We see that

$$
X_{0} \subset \bigcup_{k=2}^{\mu_{\max }} \bigcup_{i=0}^{\infty} F^{-i}\left(X_{0}^{\prime}(k)\right)
$$

Therefore, it is sufficient to show that $\nu\left(X_{0}^{\prime}(k)\right)=0$ for $k=2, \ldots, \mu_{\max }$. We fix $k=\mu_{\max }^{\prime}$ and drop it from the notation of $X_{0}^{\prime}(k)$.

Fix a point $x \in X_{0}^{\prime}$ and take an index $j$ in the infinite set $G^{\prime}:=\left\{j \in G: \mu_{j}=\mu_{\max }^{\prime}\right\}$. Set $k=\sum_{i=-1}^{j} k_{i}$. Then, by Lemma 3.3,

$$
\left|\left(F^{k}\right)^{\prime}(x)\right|^{p}>\prod_{i=j+1}^{-1}\left(\gamma_{k_{i}}\right)^{p}
$$

and hence

$$
d \nu(x)=\left|\left(F^{k}\right)^{\prime}(x)\right|^{-p} d \nu\left(F^{k} x\right)<\prod_{i=j+1}^{-1}\left(\gamma_{k_{i}}\right)^{-p} d \nu\left(F^{k} x\right) .
$$

Assuming that $\nu\left(X_{0}^{\prime}\right)$ is positive, we proceed similarly as in the proof of Proposition 4.1 (note that parameters $k_{i}, j$ depend on $x$ ),

$$
\begin{aligned}
+\infty & =\int_{X_{0}^{\prime}} \# G^{\prime}(x) d \nu(x)=\int_{X_{0}^{\prime}} \sum_{j \in G^{\prime}(x)} 1 d \nu(x) \\
& <\int_{X_{0}^{\prime}} \sum_{j \in G^{\prime}(x)} \prod_{i=j+1}^{-1}\left(\gamma_{k_{i}}\right)^{-p} d \nu\left(F^{k} x\right)
\end{aligned}
$$




$$
\begin{aligned}
& \leq \quad \int_{J} \sum_{F^{k} x=z, j \in G^{\prime}(x)} \prod_{i=j+1}^{-1}\left(\gamma_{k_{i}}\right)^{-p} d \nu(z) \\
& \leq \quad \int_{J} \sum_{x \in \mathbf{I}(z)} \prod_{i=j(x)+1}^{-1}\left(\gamma_{k_{i}(x)}\right)^{-p} d \nu(z) \\
& \leq \quad \int_{J} \sum_{j, k_{-1}, k_{-2}, \ldots, k_{j+1}}(4 \operatorname{deg} F)^{|j-1|}\left(\gamma_{k_{-1}} \gamma_{k_{-2}} \ldots \gamma_{k_{j+1}}\right)^{-p} d \nu(z) \\
& <\int_{J}\left(4 \operatorname{deg} F \sum_{k} \gamma_{k}^{-\beta}+\left(4 \operatorname{deg} F \sum_{k} \gamma_{k}^{-\beta}\right)^{2}+\left(4 \operatorname{deg} F \sum_{k} \gamma_{k}^{-\beta}\right)^{3}+\ldots\right) d \nu(z) \\
& <\int_{J}\left(\frac{1}{4}+\left(\frac{1}{4}\right)^{2}+\ldots\right) d \nu(z)=\int_{J} \frac{1}{3} d \nu(z)<+\infty .
\end{aligned}
$$

This yields a contradiction and proves the proposition.

\subsection{Conformal measures}

The notion of conformal measures was introduced to rational dynamics by D. Sullivan following an analogy with Kleinian groups, see Definition 1.3. Loosely speaking, a probabilistic measure $\nu$, supported on the Julia set, is conformal with exponent $\delta$, if its Jacobian is equal to $\left|F^{\prime}\right|^{\delta}$, i.e.

$$
d \nu(F(z))=\left|F^{\prime}(z)\right|^{\delta} d \nu(z) .
$$

D. Sullivan proved in [45] that for every Julia set there exists a conformal measure with an exponent $\delta \in(0,2]$. For hyperbolic Julia sets, there exists only one conformal measure which coincides with a normalized $\operatorname{HDim}(J)$-dimensional Hausdorff measure. In general, it is more difficult to describe analytical properties of conformal measures. For example, it is an open problem whether there exists a non-atomic conformal measure for a given rational function.

We recall that a conformal dimension $\delta_{\text {conf }}(J)$ of $J$ is defined as

$$
\delta_{\text {conf }}(J)=\inf \{\delta: \exists \delta-\text { conformal measure }\} .
$$

A simple compactness argument (see [45]) shows that infimum is attained in the definition above. The following lemma is a version of standard Patterson-Sullivan construction of conformal measures (cf. [45]):

Lemma 5.1 Let $z$ be either a critical point of the maximal multiplicity in the Julia set, or a point at a positive distance from the orbits of the critical points. Then there exists a $\delta_{\text {Poin }}(z)-$ conformal measure.

Proof: If $z$ is a critical point, then for any $q>\delta_{P o i n}(z)$ there is an atomic conformal measure supported on the preimages of $z$. To see this, normalize

$$
\sum_{n} \sum_{y \in F^{-n} z}\left|\left(F^{n}\right)^{\prime}(y)\right|^{-q} 1_{y}
$$


where $1_{y}$ is a Dirac measure at $y$, to be a probabilistic measure.

If $z$ is a point at a positive distance from the critical orbits then standard arguments of [45] apply.

Lemma 5.2 Suppose that there exist a p-conformal measure $\eta$ and a $q$-conformal measure $\nu$ which have no atoms at critical points. If $F$ satisfies the summability condition with an exponent

$$
\alpha<\frac{\max \{p, q\}}{\mu_{\max }+\max \{p, q\}}=\max \left\{\frac{p}{\mu_{\max }+p}, \frac{q}{\mu_{\max }+q}\right\},
$$

then $p=q$ and $\eta=\nu$.

Proof: If a ball $B$ of radius $r(B)$ is mapped with a bounded distortion to the large scale, i.e. $F^{n}(B)=A$, then

$$
\nu(B) \asymp\left(\frac{r(B)}{\operatorname{diam}(A)}\right)^{q} \nu(A) \asymp r(B)^{q} .
$$

Assume first that $p$ and $q$ are different, without loss of generality $p<q$. Then, by Proposition 5.1, $\nu$-almost every point goes infinitely often to the large scale with bounded distortion. This implies that for $\nu$-almost every point $z$ there is a sequence of balls $B_{j}$ of radius $R_{j} \rightarrow 0$ centered at $z$ so that

$$
\eta\left(B_{j}\right) \asymp\left(R_{j}\right)^{p}=\left(R_{j}\right)^{p-q}\left(R_{j}\right)^{q} \asymp\left(R_{j}\right)^{p-q} \nu\left(B_{j}\right) .
$$

Let $\mathcal{B}$ be a collection of all balls of radius less than $r$ which are mapped with a uniformly bounded distortion to the large scale. By the Besicovitch covering theorem (see Section 2.7 in [31]) there exists a subcollection $\mathcal{B}^{\prime}$ of $\mathcal{B}$ so that $\nu$-almost all points of $J$ are contained in $\bigcup_{B \in \mathcal{B}^{\prime}}$ and every point in $\mathbb{C}$ is covered by at most $P$ balls from $\mathcal{B}^{\prime}$. Then

$$
\begin{aligned}
\eta(J) & \geq P^{-1} \sum_{B \in \mathcal{B}^{\prime}} \eta(B) \gtrsim \sum_{B \in \mathcal{B}^{\prime}} r(B)^{p-q} \nu(B) \\
& \geq r^{p-q} \sum_{B \in \mathcal{B}^{\prime}} \nu(B) \geq r^{p-q} \nu(J)
\end{aligned}
$$

which (for sufficiently small $r$ ) contradicts the fact that $\eta(J)=\nu(J)=1$.

Hence $p=q$. If $\nu$ and $\eta$ are different probabilistic measures then their difference $\nu-\eta$ has a non-trivial positive and negative part. After normalization, $(\nu-\eta)_{-}$and $(\nu-\eta)_{+}$become $q$-conformal measures which are mutually singular. Therefore, without loss of generality, we can assume that $\nu$ and $\eta$ are mutually singular.

If $E \subset J$ is an open set then, by the Besicovitch covering theorem, we can choose a cover $\mathcal{B}^{\prime}$ of $\nu$-almost all points of $E$ such that every point in $\mathbb{C}$ is covered by at most $P$ balls and no points outside $E$ are covered. Then

$$
\eta(E) \geq P^{-1} \sum_{B \in \mathcal{B}^{\prime}} \eta(B) \asymp \sum_{B \in \mathcal{B}^{\prime}}(r(B))^{q} \asymp \sum_{B \in \mathcal{B}} \nu(B) \geq \nu(E)
$$

and consequently $\eta(E) \gtrsim \nu(E)$ for every Borel set $E$. This contradicts the mutual singularity of $\eta$ and $\nu$, and completes the proof. 
Corollary 5.1 If F satisfies the summability condition with an exponent

$$
\alpha<\frac{\delta_{\text {Poin }}(J)}{\mu_{\max }+\delta_{\text {Poin }}(J)}
$$

then

1. There is a unique $\delta_{\text {Poin }}(J)$-conformal measure. It is ergodic and non-atomic. This is the only conformal measure with no atoms at the critical points. In particular, there are no non-atomic measures with exponents different from $\delta_{\text {Poin }}(J)$.

2. There are no conformal measures with exponents less than $\delta_{\text {Poin }}(J)$, i.e. $\delta_{\text {Poin }}(J)=$ $\delta_{\text {conf }}(J)$.

3. For every $q>\delta_{\text {Poin }}(J)$ there exists an atomic $q$-conformal measure supported on the backward orbits of the critical points. Every conformal measure has no atoms at other points.

\section{Proof:}

1. By Lemma 5.1, there is a $\delta_{\text {Poin }}(J)$-conformal measure. It cannot have atoms since otherwise the corresponding Poincaré series converges and by Corollary $4.1, \delta_{\text {Poin }}(J)<$ $\delta_{\text {Poin }}(J)$. Now, uniqueness and ergodicity follow from Lemma 5.2.

2. There are no atomic measures by Corollary 4.1 and no non-atomic measures by Lemma 5.2.

3. To obtain an atomic $q$-conformal measure, $q>\delta_{P o i n}$, distribute atoms at all preimages of a critical point of the maximal multiplicity. If there is a conformal measure with an atom at a point whose orbit omits the critical points then we can easily produce a conformal measure which has no atoms at the critical points. By Lemma 5.2, the latter coincides

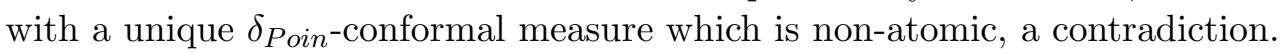

Corollary 5.1 implies Theorem 2.

\subsection{Frequency of passages to the large scale}

In this Section we give a proof of Theorem 3. Consider the set $J_{*, \epsilon}$ of all points $x \in J$ which $\epsilon$-frequently go to the large scale of size $R^{\prime}$, namely:

$$
\exists n_{j} \rightarrow \infty: F^{n_{j}} \text { univalent on } F^{-n_{j}}\left(B\left(F^{n_{j}} x, R^{\prime}\right)\right), \quad\left|\left(F^{n_{j+1}}\right)^{\prime}(x)\right|<\left|\left(F^{n_{j}}\right)^{\prime}(x)\right|^{1+\epsilon} .
$$

Note that the value of $R^{\prime}$ is already fixed and does not depend on a point. 
Proposition 5.2 Suppose that a rational function F satisfies the summability condition with an exponent

$$
\alpha<\frac{p}{\mu_{\max }+p}
$$

and for every $q>p$ there exists a point $v$ such that the Poincaré series $\Sigma_{q}(v)$ converges. If a p-conformal measure $\nu$ has no atoms at the critical points then $\nu\left(J \backslash J_{*, \epsilon}\right)=0$.

Proof: We say that a point $x$ goes to the large scale of size $R^{\prime}$ univalently at time $k$ if

$$
F^{k} \text { is univalent on } F^{-k}\left(B\left(F^{k} x, R^{\prime}\right)\right) \text {. }
$$

Assume that a point $x$ goes to the large scale at a time $m$ and apply the inductive procedure of Section 5.1 for the sequence $x, \ldots, F^{m-1}(x)$. As a result we obtain a sequence of blocks of the form $21 \ldots 13,21 \ldots 1$, or 2 (blocks of type 2 might be of zero length, meaning that we end with a type 1 block). Suppose that $y:=F^{n}(x)$ is the first point which belongs to a block of type 2 or equivalently is a terminal point of the longest sequence of the form $1 \ldots 13$ in the decomposition into blocks of the orbit $x, \ldots, F^{m-1}(x)$. By the definition of $m$, a ball of radius $R^{\prime}$ around $F^{m}(x)$ can be pulled univalently back to $x$. Hence, the same is true for a ball of radius $\Delta:=R^{\prime} /(4 M), M=\sup _{y \in J}\left|F^{\prime}(y)\right|$, around $z:=F^{m-1}(x)$. Therefore, $y \in \mathbf{I}^{\Delta}(z)=\mathbf{I}^{\Delta}(z \mid z)-$ recall that $\mathbf{I}^{\Delta}(z)$ stands for the set of the first type preimages $y \in \mathcal{H}(z, \Delta)$ of $z$, obtained in the course of the inductive procedure for $z$.

By Proposition 5.1, we already know that $\nu$-almost all points in $J$ go to the large scale infinitely often so it is sufficient to show that $\nu(X)=0$ for $X:=J_{*} \backslash J_{*, \epsilon}$.

Suppose that for every $x \in X$ there are two increasing sequences $\left\{n_{j}\right\}$ and $\left\{m_{j}:=m\left(n_{j}\right)\right\}$ such that

$$
\left|\left(F^{m_{j}}\right)^{\prime}(x)\right| \geq\left|\left(F^{n_{j}}\right)^{\prime}(x)\right|^{1+\epsilon}
$$

Therefore, $\left|\left(F^{m_{j}-n_{j}}\right)^{\prime}\left(F^{n_{j}}(x)\right)\right| \geq\left|\left(F^{n_{j}}\right)^{\prime}(x)\right|^{\epsilon}$. Denote $y=y_{j}(x):=F^{n_{j}} x$ and $z=z_{j}(x):=$ $F^{m_{j}} x$. Then

$$
\begin{aligned}
\left|\left(F^{m_{j}}\right)^{\prime}(x)\right|^{-p} & =\left|\left(F^{n_{j}}\right)^{\prime}(x)\right|^{-p}\left|\left(F^{m_{j}-n_{j}}\right)^{\prime}(y)\right|^{-p} \\
& \leq\left|\left(F^{n_{j}}\right)^{\prime}(x)\right|^{-(p+\delta)}\left|\left(F^{m_{j}-n_{j}}\right)^{\prime}(y)\right|^{-(p-\delta / \epsilon)} .
\end{aligned}
$$

Choose $\delta$ so small that $\alpha<\beta /\left(\mu_{\max }+\beta\right)$ for $\beta:=p-\delta / \epsilon$. Then, by Lemma 3.4,

$$
\sum_{y \in \mathbf{I}^{\Delta}(z)}\left|\left(F^{n(y)}\right)^{\prime}(y)\right|^{-(p-\delta / \epsilon)}<\operatorname{const}(\Delta) .
$$

By the assumptions, the Poincaré series for $q:=p+\delta$ converges for a point $v$ whose preimages are dense in the Julia set. We can choose finitely many of them, say $v_{1}, \ldots, v_{n}$, so that they are $R^{\prime} / 4$-dense in $J$ and their Poincaré series are also convergent. Now, for every point $z$ with 
$\operatorname{dist}\left(z, J_{F}\right)<R^{\prime} / 2$, there is a point $v_{j} \in B_{3 R^{\prime} / 4}(z)$. By the Koebe distortion lemma 2.1, we have that

$$
\sum_{y \in \mathbf{I I}(z)}\left|\left(F^{n}\right)^{\prime}(y)\right|^{-q} \lesssim \Sigma_{q}\left(v_{j}\right) \leq \max _{j} \Sigma_{q}\left(v_{j}\right) \lesssim \Sigma_{q}(v)<\infty
$$

and

$$
\sup _{y} \sum_{x \in \mathbf{I I}(y)}\left|\left(F^{n(x)}\right)^{\prime}(x)\right|^{-(p+\delta)}<\text { const }<\infty .
$$

Combining the estimates (21) and (22), we obtain that

$$
\begin{aligned}
& \infty \cdot \nu(X)=\int_{X} \sum_{j} 1 d \nu(x)=\int_{X} \sum_{j}\left|\left(F^{m_{j}}\right)^{\prime}(x)\right|^{-p} d \nu\left(F^{m_{j}} x\right) \\
& =\int_{J} \sum_{j, x: z=z_{j}(x)}\left|\left(F^{m_{j}(x)}\right)^{\prime}(x)\right|^{-p} d \nu(z) \\
& \leq \int_{J} \sum_{j, x: z=z_{j}(x)}\left|\left(F^{n_{j}}\right)^{\prime}(x)\right|^{-(p+\delta)}\left|\left(F^{m_{j}-n_{j}}\right)^{\prime}\left(y_{j}(x)\right)\right|^{-(p-\delta / \epsilon)} d \nu(z) \\
& \leq \int_{J} \sum_{x, j: z=z_{j}(x)}\left|\left(F^{n_{j}}\right)^{\prime}(x)\right|^{-(p+\delta)} \sum_{y: \exists x, y=y_{j}(x), z=z_{j}(x)}\left|\left(F^{m_{j}-n_{j}}\right)^{\prime}(y)\right|^{-(p-\delta / \epsilon)} d \nu(z) \\
& \leq\left(\sup _{y} \sum_{x \in \mathbf{I I}(y)}\left|\left(F^{n(x)}\right)^{\prime}(x)\right|^{-(p+\delta)}\right) \cdot \int_{J} \sum_{y \in \mathbf{I}(z)}\left|\left(F^{n(y)}\right)^{\prime}(y)\right|^{-(p-\delta / \epsilon)} d \nu(z) \\
& \leq \operatorname{const} \int_{J} \operatorname{const}(\Delta) d \nu(z)<\infty \text {. }
\end{aligned}
$$

Therefore $\nu\left(J_{*} \backslash J_{*, \epsilon}\right)=0$ and the proposition follows.

Proof of Theorem 3. Theorem 3 is a consequence of Theorem 2 and Proposition 5.2.

\section{Invariant measures}

\subsection{Polynomial summability condition}

In this Subsection we begin the proof of Theorem 4, establishing existence of an absolutely continuous invariant measure, provided the polynomial summability condition holds. We start with the geometric measure $\nu$ with exponent $\delta:=\delta_{\text {Poin }}(J)$, which exists by Theorem 2 . It is sufficient to find $Z \in L^{1}(\nu)$ such that for all $n$

$$
\frac{d \nu \circ F^{-n}}{d \nu}(z) \lesssim Z(z)
$$


In fact, any weak subsequential limit of

$$
\frac{1}{n} \sum_{k=1}^{n} d \nu \circ F^{-k},
$$

is an invariant measure, and (23) implies that its density is majorated by $Z(z)$, and hence it is absolutely continuous with respect to $\nu$.

To find $Z$ and establish (23), we proceed as follows. Given two points $y, z$ with $z=F^{n}(y)$ we define points $v=v(y, z), w=w(y, z)$ by the following construction, which is feasible for $\nu$-almost every $z$.

Since we are interested only in $\nu$-generic points, we can assume, by Proposition 5.1 , that $y$ goes to the large scale infinitely often. Let $n^{\prime}$ be the first time $n^{\prime}>n$ when $y$ goes to the large scale, and denote $w:=F^{n^{\prime}-1}(y)$. By the choice of $n^{\prime}$, the ball of radius $R^{\prime}$ around $F(w)$ can be pulled univalently back to $y$. The same is of course true for the ball of radius $\Delta:=R^{\prime} /(4 M)$ around $w, M=\sup _{y \in J}\left|F^{\prime}(y)\right|$. Now we carry out the inductive procedure from the Main Lemma 3.2 for the preimages of $w$ of order $<n^{\prime}$ till we get a block of type 2. By the definition of $n^{\prime}$, a code of the sequence $y, F(y), \ldots, z, \ldots, w$ is of the form $21 \ldots 13$. Let $v=v(y, z):=F^{l}(y)$ be the point which starts the block of type 2 (in other words, $v$ ends the blocks $1 \ldots 13)$. Note that $y \in \mathbf{I I}(v)$ and $v \in \mathbf{I}(w)=\mathbf{I}^{\Delta}(w \mid w)$. We recall that $\mathbf{I}^{\Delta}(w \mid w)$ stands for the set of first type preimages of $w$ belonging to $\mathcal{H}(w, \Delta)$ obtained in the course of the inductive procedure for $w$.

Below, we assume that $l=l(v)$ is chosen so that $v=F^{l}(y)$, and $j=j(v)=n-l$. If $F^{n}(y)=z$, we denote $n(y, z):=n$. Recalling that $\delta=\delta_{P o i n}(J)$ is the exponent of the conformal measure $\nu$, we can write

$$
\begin{aligned}
\frac{d \nu \circ F^{-n}}{d \nu}(z) & =\sum_{y \in F^{-n}(z)}\left|\left(F^{n}\right)^{\prime}(y)\right|^{-\delta} \\
& =\sum_{v: \exists y \in F^{-n}(z), v=v(y, z)} \sum_{y \in F^{-l}(v)}\left|\left(F^{l}\right)^{\prime}(y)\right|^{-\delta}\left|\left(F^{n-l}\right)^{\prime}(v)\right|^{-\delta} \\
& \leq \sum_{v: \exists y \in F^{-n}(z), v=v(y, z)}\left(\sup _{x} \sum_{y \in \mathbf{I I}(x), F^{l}(y)=x}\left|\left(F^{l}\right)^{\prime}(y)\right|^{-\delta}\right)\left|\left(F^{n-l}\right)^{\prime}(v)\right|^{-\delta} \\
& \lesssim \sum_{v: \exists y, v=v(y, z)}\left|\left(F^{n(v, z)}\right)^{\prime}(v)\right|^{-\delta}=: Z(z) .
\end{aligned}
$$

The estimate above is possible since for a fixed $n$ and $z$ every point $v \in F^{-j} z$ is counted only if it is $v(y, z)$ for some $y \in F^{-n} z$, and in this case $l=n-j$ is fixed (and independent of $y$ ). However, once the summation is done, $n$ disappears from the estimate and does not figure in the definition of $Z$. Note also that summation set satisfies

$$
\left\{v: \exists n, y \in F^{-n}(z), v=v(y, z)\right\} \subset\left\{v: \exists w, y \in \mathbf{I}(w), y \in F^{-n}(z) \cap F^{-m}(w), m \geq n\right\} .
$$


Thus it suffices to prove that $Z \in L^{1}(\nu)$, which we can do by writing

$$
\begin{aligned}
\int Z(z) d \nu(z) & =\int \sum_{v: \exists y, v=v(y, z)}\left|\left(F^{n(v, z)}\right)^{\prime}(v)\right|^{-\delta} d \nu(z) \\
& \leq \int \sum_{v, z: \exists y, v=v(y, z), w=w(y, z)}\left|\left(F^{n(v, w)}\right)^{\prime}(v)\right|^{-\delta} d \nu(w) \\
& \leq \int \sum_{v \in \mathbf{I}(w)} n(v, w)\left|\left(F^{n(v, w)}\right)^{\prime}(v)\right|^{-\delta} d \nu(w) \\
& \lesssim \int \sum_{n} n \sum_{i, k_{1}, \ldots, k_{i}: k_{1}+\ldots+k_{i}=n}\left(\gamma_{k_{1}} \ldots \gamma_{k_{i}}\right)^{-\delta} \\
& \lesssim \int \sum_{n} n \gamma_{n}^{-\delta}<\infty,
\end{aligned}
$$

the last inequality being true since $F$ satisfies the polynomial summability condition with an exponent $\alpha<\delta /\left(\delta+\mu_{\max }\right)$. We also use above that for given $v$ and $w$ there are at most $n(v, w)$ possible choices of $z$, namely $v, F(v), \ldots, F^{n(v, w)-1}(v)=F^{-1}(w)$. This concludes the proof of the existence of an absolutely continuous invariant measure.

\subsection{Ergodic properties}

In this Section we complete the proof of Theorem 4, establishing that an absolutely continuous invariant measure is unique, ergodic, mixing, exact, has positive entropy and Lyapunov exponent. We do not require the polynomial summability condition of Theorem 4: it is sufficient to assume the corresponding summability condition and existence of an absolutely continuous invariant measure.

If an absolutely continuous invariant measure exists, its ergodicity and uniqueness follow immediately from the ergodicity of the geometric measure asserted by Theorem 2 .

Lyapunov exponents. A Lyapunov exponent of $F$ at $z$ is defined as

$$
\chi(z):=\lim _{n \rightarrow \infty} \frac{1}{n} \log \left|\left(F^{n}\right)^{\prime}(z)\right|,
$$

provided that the limit exists. A Lyapunov exponent of an invariant measure $\sigma$ is defined as $\chi_{\sigma}=\int \log \left|F^{\prime}\right| d \sigma$. Birkhoff's ergodic theorem implies that if $\sigma$ is ergodic then for almost every point $z$ with respect to $\sigma$ the Lyapunov exponent $\chi(z)$ exists and is equal to $\chi_{\sigma}$. The next lemma is based on standard reasoning (see e.g. [11]).

Lemma 6.1 Let $\nu$ be a geometric measure of a rational function $F$ which satisfies the summability condition with an exponent

$$
\alpha<\frac{\delta_{\text {Poin }}(J)}{\delta_{\text {Poin }}(J)+\mu_{\max }} .
$$


Suppose that $\sigma$ is an absolutely continuous invariant measure with respect to $\nu$. Then $\sigma$ has positive entropy and Lyapunov exponent and for almost every point $z$ with respect to $\nu$,

$$
\chi(z)=\int \log \left|F^{\prime}\right| d \sigma>0 .
$$

Proof: The entropy is given by the formula $h_{\sigma}=\int \log \mathrm{Jac}_{\sigma} d \sigma$ where the Jacobian is defined as the Radon-Nikodym derivative: $\operatorname{Jac}_{\sigma}:=d \sigma \circ F / d \sigma$. The latter is always $\geq 1$ since $\sigma$ is invariant. In our case for sufficiently small sets $A$ which do not contain the critical points of $F$ we can write

$$
\left.\mathrm{Jac}_{\sigma}\right|_{A} \asymp \frac{\sigma(F(A))}{\sigma(A)} \asymp \frac{\nu(F(A))}{\nu(A)}>0,
$$

and hence

$$
1=\sum_{y \in F^{-1} F(y)} \frac{1}{\operatorname{Jac}_{\sigma}(y)}>\frac{1}{\operatorname{Jac}_{\sigma}(y)}
$$

for $\sigma$-almost every y. We conclude that $\sigma$-almost everywhere $\mathrm{Jac}_{\sigma}>1$ and hence entropy of $\sigma$ is positive. Since $\sigma$ is invariant and ergodic, the remaining statements follow from [30].

Exactness. Recall that a measure preserving endomorphism $F$ is called mixing if for every two measurable sets $A$ and $B$

$$
\lim _{n \rightarrow \infty} \sigma\left(A \cap T^{-n}(B)\right)=\sigma(A) \sigma(B) .
$$

A measure preserving endomorphism $F$ is exact if for every measurable $A, 0<\nu(A)<1$, there is no sequence of sets $A_{n}$ so that $A=F^{-n}\left(A_{n}\right)$.

Lemma 6.2 Suppose that $F$ satisfies the summability condition with an exponent

$$
\alpha<\frac{\delta_{\text {Poin }}(J)}{\delta_{\text {Poin }}(J)+\mu_{\max }},
$$

and has an absolutely continuous invariant measure $\sigma$. Then

$$
\limsup _{n \rightarrow \infty} \sigma\left(F^{n}(A)\right)=1
$$

for every measurable set $A$ of positive $\sigma$-measure, and hence $F$ is exact and mixing.

Proof: The proof that exactness implies mixing can be found in [48]. Also it is clear that ( $\sigma$ is absolutely continuous with respect to an ergodic $\nu$ ) it is sufficient to prove the same statement for $\nu: \lim \sup _{n \rightarrow \infty} \nu\left(F^{n}(A)\right)=1$.

By Proposition 5.1, there exists $R^{\prime}>0$ so that for almost every point $z \in J$ with respect to $\sigma$ there is a sequence of integers $n_{j}$ and sequences of balls $B_{r_{j}}(z)$ and topological disks $D_{j}\left(F^{n_{j}}(z)\right) \supset B_{R^{\prime}}\left(F^{n_{j}}(z)\right)$ so that $F^{n_{j}}: B_{r_{j}}(z) \mapsto D_{j}\left(F^{n_{j}}(z)\right)$ is a univalent function with 
bounded distortion. Let $z$ be a density point of $A$ with respect to $\nu$. The bounded distortion of $F^{n_{j}}$ implies that for every $\epsilon>0$ there exist $j$ so that

$$
\frac{\nu\left(A \cap D_{j}\left(F^{n_{j}}(z)\right)\right)}{\nu\left(D_{j}\left(F^{n_{j}}(z)\right)\right)} \geq(1-\epsilon) \frac{\nu\left(A \cap B_{r_{j}}(z)\right)}{\nu\left(B_{r_{j}}(z)\right)} \geq 1-2 \epsilon .
$$

By compactness, there exists $N=N\left(R^{\prime}\right)$ such that every disk $B_{R^{\prime}}(y), y \in J$, is mapped onto $J$ by $F^{N}$. Hence,

$$
\lim _{j \rightarrow \infty} \nu\left(F^{n_{j}+N}(A)\right) \geq \lim _{j \rightarrow \infty} \frac{\nu\left(A \cap D_{j}\left(F^{n_{j}}(z)\right)\right)}{\nu\left(D_{j}\left(F^{n_{j}}(z)\right)\right)} \nu(J)=1,
$$

and the lemma follows.

This concludes the proof of Theorem 4 . 


\section{Part II}

\section{Geometry, rigidity, perturbations}

\section{$7 \quad$ Fractal structure}

In this Section we will prove that the geometry of the Julia sets satisfying appropriate summability conditions is effectively fractal and self-similar. Namely, every sufficiently small ball shrinks under the pull-backs and hence its geometry is infinitely many times reproduced at different scales. Moreover, it is "usually" (i.e. around most points and for most scales) reproduced with bounded distortion.

\subsection{Average contraction of preimages}

Proposition 7.1 Suppose that a rational function $F$ satisfies the summability condition with an exponent

$$
\alpha<\frac{2}{\mu_{\max }+2}
$$

and the Julia set is not the whole sphere. Then there is $p<2$ such that for every sufficiently small ball $B$ with center on the Julia set

$$
\sum_{n} \sum_{F^{-n}}\left(\operatorname{diam}\left(F^{-n} B\right)\right)^{p} \#\left(F^{n}, F^{-n} B\right)<\infty
$$

where $\#\left(F^{n}, F^{-n} B\right)$ denotes the degree of $F^{n}$ restricted to the connected component $F^{-n} B$ of the preimage of $B$ under $F^{-n}$.

We continue to work with sequences $\left\{\alpha_{n}\right\},\left\{\gamma_{n}\right\},\left\{\delta_{n}\right\}$ of Lemma 2.2. To control the diameters we will need a new decomposition procedure.

Local Analysis. First we prove the analogues of Lemma 2.3 and Lemma 2.5.

Lemma 7.1 Suppose that

1) Shrinking neighborhoods $U_{k}$ for $B_{4 r}(z), 1 \leq k<n$, avoid critical points and $(r)^{\mu_{1}}<R$,

2) a critical point $c_{2} \in U_{n}$,

3) a critical point $c_{1} \in B_{r}(z)$.

To simplify notation set $\mu_{i}:=\mu\left(c_{i}\right), r_{2}:=\left(\right.$ diam $\left.\left(U_{n}\right)\right)$ and, for consistency, $r_{1}:=r$. Then

$$
\left(r_{2}\right)^{\mu_{2}}<\left(r_{1}\right)^{\mu_{1}}\left(\gamma_{n}\right)^{-\mu_{\max }}
$$

in particular, $\left(r_{2}\right)^{\mu_{2}}<R$. 
Proof: First note, that $F^{n} c_{2} \in B_{4 r \Delta_{n}}$, hence

$$
\operatorname{dist}\left(F^{n} c_{2}, c_{1}\right) \leq \operatorname{dist}\left(F^{n} c_{2}, z\right)+\operatorname{dist}\left(z, c_{1}\right) \leq 5 r_{1}<5 R^{1 / \mu_{\max }}
$$

and by the choice of $R$ we have $\left|F^{\prime}\left(F^{n} c_{2}\right)\right| \stackrel{M}{\asymp} \operatorname{dist}\left(F^{n} c_{2}, c_{1}\right)^{\mu_{1}-1}$. Therefore,

$$
\left|\left(F^{n-1}\right)^{\prime}\left(F c_{2}\right)\right|^{-1} \leq M \operatorname{dist}\left(F^{n} c_{2}, c_{1}\right)^{\mu_{1}-1}\left|\left(F^{n}\right)^{\prime}\left(F c_{2}\right)\right|^{-1} \leq \frac{M\left(5 r_{1}\right)^{\mu_{1}-1}}{\sigma_{n}}
$$

We recall that $U_{n} \supset U_{n-1}^{\prime}=F\left(U_{n}\right)$. By the Koebe distortion theorem (see Lemma 2.1) applied to the conformal map $F^{-(n-1)}: B_{4 r_{1} \Delta_{n-1}}(z) \rightarrow U_{n-1}$ we obtain that

$$
\begin{aligned}
\operatorname{diam}\left(U_{n-1}^{\prime}\right) & \leq 2 \frac{\left(1-\delta_{n}\right)\left(2-\delta_{n}\right)}{\delta_{n}} \Delta_{n-1} 4 r_{1}\left|\left(F^{n-1}\right)^{\prime}\left(F c_{2}\right)\right|^{-1} \\
& \leq \frac{16 r_{1}}{\delta_{n}} \frac{M\left(5 r_{1}\right)^{\mu_{1}-1}}{\sigma_{n}} \\
& \leq 16^{\mu_{\max }} M\left(\alpha_{n}\right)^{-2}\left(r_{1}\right)^{\mu_{1}}\left(\gamma_{n}\right)^{-\mu_{\max }} \\
& \leq\left(r_{1}\right)^{\mu_{1}}\left(\gamma_{n}\right)^{-\mu_{\max }}\left(\alpha_{n}\right)^{-1}
\end{aligned}
$$

The last inequality is true by our choice of $\alpha_{n}$ and $R$, see condition (ii) in Section 2.3. In particular, diam $\left(U_{n-1}^{\prime}\right)<\left(r_{1}\right)^{\mu_{1}}<R$ and again by condition (i) of Section 2.3 we have that

$$
\begin{aligned}
\left(r_{2}\right)^{\mu_{2}} & \leq M \operatorname{diam}\left(U_{n-1}^{\prime}\right) \\
& \leq M\left(r_{1}\right)^{\mu_{1}}\left(\gamma_{n}\right)^{-\mu_{\max }}\left(\alpha_{n}\right)^{-1} \\
& \leq\left(r_{1}\right)^{\mu_{1}}\left(\gamma_{n}\right)^{-\mu_{\max }}<R
\end{aligned}
$$

which completes the proof.

\section{Lemma 7.2 Suppose that}

1) shrinking neighborhoods $U_{k}$ for $B_{4 r}(z), 1 \leq k<n$, avoid critical points and $r<R^{\prime}$,

2) a critical point $c_{2} \in U_{n}$.

Set $\mu_{2}:=\mu\left(c_{2}\right)$ and $r_{2}:=\left(\operatorname{diam}\left(U_{n}\right)\right)$. For consistency, put $r_{1}:=r$. Then

$$
\left(r_{2}\right)^{\mu_{2}}<\left(\gamma_{n}\right)^{-\mu_{\max }}
$$

and $\left(r_{2}\right)^{\mu_{2}}<R$. 
Proof: Applying the Koebe distortion Lemma 2.1 we obtain that

$$
\begin{aligned}
\operatorname{diam}\left(U_{n-1}^{\prime}\right) & \leq 2 \frac{\left(1-\delta_{n}\right)\left(2-\delta_{n}\right)}{\delta_{n}} \Delta_{n-1} 4 r_{1}\left|\left(F^{n-1}\right)^{\prime}\left(F c_{2}\right)\right|^{-1} \\
& \leq \frac{16 r_{1}}{\delta_{n}} \frac{\sup \left|F^{\prime}\right|}{\sigma_{n}} \\
& \leq 16 R^{\prime} \sup \left|F^{\prime}\right|\left(\alpha_{n}\right)^{-2}\left(\gamma_{n}\right)^{-\mu_{\max }} \\
& \leq R M^{-1}\left(\gamma_{n}\right)^{-\mu_{\max }} \leq R
\end{aligned}
$$

The last inequality is true by the choice of $R^{\prime}$. Particularly, $U_{n-1}^{\prime}$ is close to $F c_{2}$ and

$$
\begin{aligned}
\left(r_{2}\right)^{\mu_{2}} & \leq M \operatorname{diam}\left(U_{n-1}^{\prime}\right) \\
& \leq M M^{-1} R\left(\gamma_{n}\right)^{-\mu_{\max }} \\
& =R\left(\gamma_{n}\right)^{-\mu_{\max }} .
\end{aligned}
$$

Proof of Proposition 7.1. Let $z$ be a point from the Julia set and fix an inverse branch of $F^{-n}$ so that $F^{-n}(z) \mapsto \ldots \mapsto F^{-1}(z) \mapsto z$. Next, take a ball $B=B_{r_{1}}(z)$ of sufficiently small radius $r_{1}<R^{\prime}$ and consider the shrinking neighborhoods for the 4 times larger ball $B_{4 r_{1}}(z)$. Let $k_{1}$ be the first time when $U_{k_{1}}$ catches a critical point $c_{2}$. Then, by Lemma 7.2 , $r_{2}:=\operatorname{diam}\left(F^{-k_{1}} B_{r_{1}}\right)$, we have that

$$
\left(r_{2}\right)^{\mu_{2}}<\left(\gamma_{k_{1}}\right)^{-\mu_{\max }}
$$

Consider now the shrinking neighborhoods for the ball $B_{4 r_{2}}\left(z_{2}\right)$ with $z_{2}:=F^{-k_{1}} z$. Let $k_{2}$ be the first time when $U_{k_{2}}$ hits a critical point $c_{3}$. Again, by Lemma 7.1, $r_{3}:=\operatorname{diam}\left(F^{-k_{2}} B_{r_{2}}\right)$, we obtain that

$$
\left(r_{3}\right)^{\mu_{3}}<\left(r_{2}\right)^{\mu_{2}}\left(\gamma_{k_{2}}\right)^{-\mu_{\max }}
$$

We continue in the same fashion, taking shrinking neighborhoods for $B_{4 r_{3}}\left(z_{3}\right)$ with $z_{3}:=$ $F^{-k_{2}} z_{2}$, and so on. Observe, that during the construction we always have

$$
F^{-\left(k_{1}+k_{2}+\ldots+k_{j}\right)} B \subset F^{-\left(k_{2}+\ldots+k_{j}\right)} B_{r_{2}}\left(z_{2}\right) \subset \ldots \subset B_{r_{j+1}}\left(z_{j+1}\right),
$$

and there is a bound for the degree:

$$
\#\left(F^{\left(k_{1}+k_{2}+\ldots+k_{j}\right)}, F^{-\left(k_{1}+k_{2}+\ldots+k_{j}\right)} B\right) \leq\left(\mu_{\max }\right)^{j}
$$

We can repeat the above construction until we meet a ball $B_{4 r_{l}}\left(F^{-k_{l-1}} z\right)$ whose shrinking neighborhoods do not contain critical points. This means that the ball $B_{2 r_{l}}\left(z_{l}\right)$ can be pulled back univalently along the considered branch. We will call $z_{l}$ a terminal point. In more general notation, $y:=z_{l}$ with parameters $r(y)=r_{l}, l(y)=l, c_{y}=c_{l}$. 
Now, we look at the backward orbit of $z$ for all possible inverse branches of $F$ and denote by $\mathcal{Y}(z)$ the set of all terminal points.

By the Koebe distortion theorem,

$$
\begin{aligned}
\operatorname{diam}\left(F^{-n} B\right) & <\operatorname{diam}\left(F^{-m} B_{r_{l}}\left(z_{l}\right)\right) \\
& <16\left|\left(F^{-m}\right)^{\prime}\left(z_{l}\right)\right| r_{l} \\
& =16 r_{l}\left|\left(F^{m}\right)^{\prime}(x)\right|^{-1},
\end{aligned}
$$

where $m=\left(n-k_{1}-\ldots-k_{l-1}\right)$ and $x=F^{-m} z_{l}=F^{-n} z$. Note, that $x \in \mathcal{H}\left(z_{l}, r_{l}\right)$ in the terminology of Proposition 4.1, and $\#\left(F^{n}, F^{-n} B\right) \leq\left(\mu_{\max }\right)^{l-1}$.

Now, using the result of Proposition 4.1 we can expand (for $p<2$ close to 2)

$$
\begin{aligned}
\sum_{n} \sum_{F^{-n}} & \left(\operatorname{diam}\left(F^{-n} B\right)\right)^{p} \#\left(F^{n}, F^{-n} B\right) \\
& <\sum_{y \in \mathcal{Y}(z)} \sum_{x \in \mathcal{H}(y, r(y))} 16^{p}(r(y))^{p}\left|\left(F^{n(x)}\right)^{\prime}(x)\right|^{-p} \#\left(F^{n}, F^{-n} B\right) \\
& <\sum_{y \in \mathcal{Y}(z)} 16^{p}(r(y))^{p} C(r(y))^{p\left(\mu\left(c_{y}\right) / \mu_{\max }-1\right)} \#\left(F^{n}, F^{-n} B\right) \\
& <16^{p} C \sum_{y \in \mathcal{Y}(z)}(r(y))^{p \mu\left(c_{y}\right) / \mu_{\max }}\left(\mu_{\max }\right)^{l(y)-1} \\
& <16^{p} C \sum_{y \in \mathcal{Y}(z)}\left(\gamma_{k_{l-1}-\mu_{\max }} \ldots \gamma_{k_{1}}^{-\mu_{\max }}\right)^{p / \mu_{\max }}\left(\mu_{\max }\right)^{l-1} \\
& <16^{p} C \sum_{l, k_{1}, \ldots, k_{l-1}}(2 \operatorname{deg} F)^{l}\left(\gamma_{k_{1}} \ldots \gamma_{k_{l-1}}\right)^{-p}\left(\mu_{\max }\right)^{l-1} \\
\leq & 16^{p} C \sum_{l}\left(2 \operatorname{deg} F \mu_{\max } \sum_{k} \gamma_{k}^{-p}\right)^{l} \\
< & C 16^{p} \sum_{l}\left(\frac{1}{2}\right)^{l}=C 16^{p}<\infty
\end{aligned}
$$

which proves Proposition 7.1.

Note, that substuting into the last formula Lemma 3.4 (instead of Proposition 4.1), we can arrive at a better estimate (where the sum is taken only over some preimages):

Corollary 7.1 Taking $\beta=\mu_{\max } \alpha /(1-\alpha)$ and using the notation above, we get

$$
\sum_{y \in \mathcal{Y}(z)} \sum_{x \in \mathbf{I}^{r(y)}(y)}\left(\operatorname{diam}\left(F^{-n(x, z)} B\right)\right)^{\beta}<\infty .
$$




\subsection{Contraction of preimages}

Proposition 7.2 Suppose that a rational function $F$ satisfies the summability condition with an exponent

$$
\alpha \leq 1
$$

Then there exist a positive sequence $\left\{\tilde{\omega}_{n}\right\}$, summable with an exponent $-\beta:=-\frac{\mu_{\max } \alpha}{1-\alpha}$ :

$$
\sum_{n}\left(\tilde{\omega}_{n}\right)^{-\beta}<\infty
$$

such that for every sufficiently small (of radius less than $R^{\prime}$ ) ball $B$ centered on the Julia set, every $n$, and every branch of $F^{-n}$ we have

$$
\operatorname{diam}\left(F^{-n} B\right)<\left(\tilde{\omega}_{n}\right)^{-1}
$$

Remark 7.1 The proof of Proposition 7.2 given below will actually imply that

$$
\operatorname{diam}\left(F^{-n} B\right)<\operatorname{const}\left(\tilde{\omega}_{n}\right)^{-1}(\operatorname{diam}(B))^{1 / \mu_{\max }} .
$$

Also, for any periodic point $z: F^{k}(z)=z$, by the proposition above we can find such $n$ that for the branch of $F^{-k n}$, fixing $z$, and a small ball $B(z, \rho)$ one has

$$
F^{-n k} B(z, \rho) \subset B(z, \rho / 2) \text {. }
$$

By a standard use of the Schwartz lemma, the latter implies $\left|\left(F^{k}\right)^{\prime}(z)\right|>1$, and we arrive at the following

Corollary 7.2 Under the assumptions as above, F has no Cremer points.

Proof: To prove Proposition 7.2, take a ball $B_{r}(z)$ of a small radius $r_{1}<R^{\prime}$ and proceed as in the proof of Proposition 7.1 - we preserve the notation. Then, with the help of Proposition 2.1, (the sequence $\left\{\omega_{n}\right\}$ was constructed there) we obtain that

$$
\begin{aligned}
\operatorname{diam}\left(F^{-n} B\right) & <\operatorname{diam}\left(F^{-m} B_{r_{l}}\left(z_{l}\right)\right) \\
& <16\left|\left(F^{-m}\right)^{\prime}\left(z_{l}\right)\right| r_{l} \\
& <16 r_{l}\left(r_{l}\right)^{\mu\left(c_{l}\right) / \mu_{\max }-1}\left(\omega_{m}\right)^{-1} \\
& <16\left(r_{l}\right)^{\mu\left(c_{l}\right) / \mu_{\max }}\left(\omega_{m}\right)^{-1} \\
& <16\left(\left(\gamma_{k_{1}}\right)^{-\mu_{\max }} \ldots\left(\gamma_{k_{l}}\right)^{-\mu_{\max }}\right)^{1 / \mu_{\max }}\left(\omega_{m}\right)^{-1} \\
& <16\left(\gamma_{k_{1}}\right)^{-1} \ldots\left(\gamma_{k_{l}}\right)^{-1}\left(\omega_{m}\right)^{-1},
\end{aligned}
$$

where $k_{1}+\ldots+k_{l}+m=n$.

It means that setting

$$
\tilde{\omega}_{n}:=\inf \left\{\gamma_{k_{1}} \ldots \gamma_{k_{l}} \omega_{m} / 16: k_{1}+\ldots+k_{l}+m=n\right\}
$$


we have that

$$
\operatorname{diam}\left(F^{-n} B\right)<\left(\tilde{\omega}_{n}\right)^{-1} .
$$

On the other hand (for $-\beta:=-\frac{\mu_{\max } \alpha}{1-\alpha}$ )

$$
\begin{aligned}
\sum_{n}\left(\tilde{\omega}_{n}\right)^{-\beta} & <16^{\beta}\left(\sum_{m}\left(\omega_{m}\right)^{-\beta}\right) \cdot \sum_{l=0}^{\infty}\left(\sum_{k}\left(\gamma_{k}\right)^{-\beta}\right)^{l} \\
& <16^{\beta} \sum_{m}\left(\omega_{m}\right)^{-\beta} \cdot \sum_{l=0}^{\infty}\left(\frac{1}{2}\right)^{l}=16^{\beta} 2 \sum_{m}\left(\omega_{m}\right)^{-\beta}<\infty .
\end{aligned}
$$

which completes the proof of Proposition 7.2.

\subsection{Most points go to large scale infinitely often}

We will prove that the Hausdorff dimension of points which do not "go to a large scale infinitely often" is small provided $F$ satisfies the summability condition. This should be compared with Proposition 5.1 where it is shown that most points go to a large scale infinitely often with respect to conformal measure.

The definition of the subset of points in $J$ which infinitely often go to the large scale of size $R^{\prime} / 2$ is as follows:

$$
J_{*}:=\left\{z \in J: \exists n_{j} \rightarrow \infty \text {, with } F^{n_{j}} \text { univalent on } F^{-n_{j}}\left(B\left(F^{n_{j}} x, R^{\prime} / 2\right)\right)\right\} .
$$

Note that the value of $R^{\prime}$ is already fixed and does not depend on a point.

Proposition 7.3 Suppose that a rational function $F$ satisfies the summability condition with an exponent $\alpha<1$, then

$$
\operatorname{HDim}\left(J \backslash J_{*}\right) \leq \frac{\mu_{\max } \alpha}{1-\alpha} .
$$

Proof: The proof is a modification of the proof of Proposition 5.1. Denote $\beta:=\frac{\mu_{\max } \alpha}{1-\alpha}$.

Take a finite cover $\left\{B_{j}: j=1, \ldots, K\right\}$ of the Julia set by balls of radii $R^{\prime} / 2$ centered at points $w_{j} \in J$. For every $x \in J$ and every $k \in \mathbb{N}$, we decompose the sequence $x, \ldots, F^{k}(x)$ into blocks of new types $1^{*}, 3^{*}$, and blocks of old types $1,2,3$. An inductive procedure ascribing a code to the sequence $x, \ldots, F^{k}(x)$ will be defined only for preimages of the center of the ball $B_{j} \ni F^{k}(x)$. By the definition, the sequence $x, \ldots, F^{k}(x)$ inherits the code of the corresponding sequence of preimages $F^{-k}\left(w_{j}\right), \ldots, w_{j}$.

We start by defining blocks of type $1^{*}$ and $3^{*}$ for the preimages of $w_{j}$. To this aim we invoke the inductive procedure from Proposition 7.1. Namely, we start by picking a ball $B_{j} \subset$ $B_{R^{\prime}}\left(F^{k}(x)\right)$, denoting $z_{1}:=w_{j}, r_{1}:=R^{\prime} / 2$, and considering the shrinking neighborhoods for the 4 times larger ball $B_{4 r_{1}}(z)$. Let $k_{1}$ be the first time when $U_{k_{1}}$ hits a critical point $c_{2}$. We set $r_{2}:=\operatorname{diam}\left(F^{-k_{1}} B_{r_{1}}\right), z_{2}:=F^{-k_{1}}\left(z_{1}\right)$, and proceed by induction. The construction is repeated 
until we meet a ball $B_{4 r_{l}}\left(F^{-k_{l-1}} z\right)$ whose shrinking neighborhoods do not contain critical points. This means that the ball $B_{2 r_{l}}\left(z_{l}\right)$ can be pulled back univalently along the corresponding branch of $F^{-k+\left(k_{1}+\ldots+k_{l}\right)}$. Summarizing, our construction leads to a decomposition of the sequence $z_{l}, F\left(z_{l}\right), \ldots, z_{1}$ into blocks $1^{*} \ldots 1^{*} 3^{*}$. We see that the symbol $3^{*}$ stands for the initial sequence of preimages of type 3 with $r=2 R^{\prime}$ (see Definition 2.3). The terminal point of the type $3^{*}$ sequence is $z_{2}$. After, only type $1^{*}$ sequences are allowed with terminal points $z_{3}, \ldots, z_{l}$, respectively.

Having defined $z_{l}$, we apply to $F^{-k+k_{1}+\ldots+k_{l-1}}\left(z_{l}\right), \ldots, z_{l}$ the inductive procedure with a stopping rule (see Section 5.1). This yields a decomposition of the sequence into blocks of the form $21 \ldots 3$ or 2. Finally, we can represent the orbit $F^{-k}\left(z_{1}\right), \ldots, z_{1}$, as a sequence of blocks $21 \ldots 111^{*} 1^{*} \ldots 1^{*} 3^{*}$. By our convention, the orbit $x, \ldots, F^{k}(x)$ has the same decomposition. Note that if we have no blocks of type $1^{*}$, i.e. $z_{l}$ coincides with $F^{-k}\left(w_{j}\right)$, then the ball of radius $R^{\prime}$ can be pulled back univalently along the corresponding branch of $F^{-k}$, and we have no blocks 1 either. This means that the sequence $x, \ldots, F^{k}(x)$ is encoded as 2 . Note also that the corresponding endpoints of blocks from the orbits $x, \ldots, F^{k}(x)$ and $F^{-k}\left(z_{1}\right), \ldots, z_{1}$ are $R$-close to each other.

Following the proof of Proposition 7.1, we denote by $\mathcal{Y}\left(w_{j}\right)$ the set of all possible terminal points $z_{l}$ for all inverse branches of $F$ defined on the ball $B_{j}$. Introducing more general notation, we set $y:=z_{l}, r(y)=r_{l}, l(y)=l$, and $c_{y}=c_{l}$.

Denote by $\mathcal{C}_{x}$ the set of all codes obtained for $x$. If for some point $x$ we get infinitely many different type 2 sequences then $x$ must belong to $J_{*}$. Indeed, a type 2 sequence means that an $R^{\prime}$-ball around a point $R^{\prime} / 2$-close to some image of $x$ can be pulled back univalently. Hence, the same is true for $R^{\prime} / 2$-ball around the image of $x$.

Therefore, if $x \in J \backslash J_{*}$ then $x$ is a terminal point of an infinite number of sequences $211 \ldots 111^{*} 1^{*} \ldots 1^{*} 1^{*} 3^{*}$ with only a finite number of choices for type 2 blocks. Let $k(x)$ be a minimal number for which infinitely many sequences from $\mathcal{C}_{x}$ have the same type 2 block of length $k(x)$. Denote $X_{k}:=\{x: k(x)=k\}$ and observe that the sets $\left\{X_{k}: k=0,1, \ldots\right\}$ form a countable partition of $J \backslash J_{*}$.

Obviously, for any Borel set $X \subset J$ we have $\operatorname{HDim}\left(F^{k} X\right)=\operatorname{HDim}(X)=\operatorname{HDim}\left(F^{-k} X\right)$. Since $F^{k}\left(X_{k}\right) \subset X_{0}$ and consequently $J \backslash J_{*} \subset \cup_{k} F^{-k}\left(X_{0}\right)$, it is sufficient to prove that $\operatorname{HDim}\left(X_{0}\right) \leq \mu_{\max } \alpha /(1-\alpha)$.

Every point $x \in X_{0}$ must be a terminal point for infinitely many different subsequences of the form $1 \ldots 11^{*} \ldots 1^{*} 3^{*}$, containing at least one block $1^{*}$. Thus every point $x \in X_{0}$ is covered by infinitely many preimages

$$
F^{-n\left(v, w_{j}\right)}\left(B_{j}\right), \quad j=1, \ldots, K, \quad y \in \mathcal{Y}_{j}, \quad v \in \mathbf{I}^{r(y)}(y) .
$$

Corollary 7.1 implies that for every $j=1, \ldots, K$ and $\beta=\mu_{\max } \alpha /(1-\alpha)$,

$$
\sum_{y \in \mathcal{Y}\left(w_{j}\right)} \sum_{v \in \mathbf{I}^{r(y)}(y)}\left(\operatorname{diam}\left(F^{-n\left(v, w_{j}\right)} B_{j}\right)\right)^{\beta}<\infty .
$$

We conclude that $\operatorname{HDim}\left(X_{0}\right) \leq \frac{\mu_{\max } \alpha}{1-\alpha}$ which proves the proposition. 


\section{Dimensions and conformal measures}

\subsection{Fractal dimensions}

First we will remind the definitions of various dimensions, used in this paper. For properties of the Hausdorff and Minkowski measures, contents, and dimensions one can consult the monographs [31] and [15].

Assume that we are given a compact subset $K$ of the complex plane (or a complex sphere with the spherical metric).

Definition 8.1 For positive $\delta$ the Hausdorff measure $\mathcal{H}_{\delta}$ is defined by

$$
\mathcal{H}_{\delta}(K):=\lim _{\rho \rightarrow 0} \inf _{\mathcal{B}_{\rho}} \sum_{B \in \mathcal{B}_{\rho}} r(B)^{\delta},
$$

the infimum taken over all covers $\mathcal{B}_{\rho}=\{B\}$ of the set $K$ by balls $B$ of radii $r(B) \leq \rho$.

Usually the measure above is normalized by some factor, depending on $\delta$, but this is not necessary for our purposes.

It is easy to show that there exists some number $\delta^{\prime} \in[0,2]$, such that $\mathcal{H}_{\delta}(K)$ is infinite for $\delta<\delta^{\prime}$ and zero for $\delta>\delta^{\prime}$. The latter is called the Hausdorff dimension:

Definition 8.2 The Hausdorff dimension of a set $K$ is defined by

$$
\operatorname{HDim}(K):=\inf \left\{\delta: \mathcal{H}_{\delta}(K)=0\right\} .
$$

The Hausdorff dimension of a Borel measure $\nu$ is defined as the infimum of the dimensions of its Borel supports:

$$
\operatorname{HDim}(\nu):=\inf \left\{\operatorname{HDim}(E): E \text { is Borel and } \nu\left(E^{c}\right)=0\right\} .
$$

The upper and lower Minkowski dimensions can be defined similarly using the corresponding Minkowski contents. Equivalently, one can take a shortcut and define them as follows:

Definition 8.3 Let $N(K, \rho)$ be the minimal number of the balls of radius $\rho$ needed to cover $K$. The upper and lower Minkowski dimensions are defined as

$$
\begin{aligned}
& \overline{\operatorname{MDim}}(K):=\limsup _{\rho \rightarrow 0} \frac{\log N(K, \rho)}{\log 1 / \rho}, \\
& \underline{\operatorname{MDim}}(K):=\liminf _{\rho \rightarrow 0} \frac{\log N(K, \rho)}{\log 1 / \rho}
\end{aligned}
$$

If those dimensions coincide, their common value is called the Minkowski dimension $\operatorname{MDim}(K)$.

Remark 8.1 Since we restricted ourselves to a smaller collection of coverings, than in the definition of the Hausdorff measure, one clearly has

$$
\operatorname{HDim}(K) \leq \underline{\operatorname{MDim}}(K) \leq \overline{\operatorname{MDim}}(K),
$$

for arbitrary compact set $K$. 
In the absence of the dynamics, the Whitney exponent can be regarded as a substitute for the Poincaré exponent. One can decompose domain $\Omega:=\mathbb{C} \backslash K$ in the complex plane into a collection $\left\{Q_{j}\right\}$ of non-overlapping dyadic squares so that $\operatorname{dist}\left(Q_{j}, K\right) \asymp \operatorname{diam}\left(Q_{j}\right)$ up to a constant of 4 (consult [44] for this classical fact, the Whitney decomposition).

Definition 8.4 Whitney exponent is defined as an exponent of convergence

$$
\delta_{W h i t}(K):=\inf \left\{\delta: \sum_{Q_{j}: \operatorname{diam}\left(Q_{j}\right) \leq 1} \operatorname{diam}\left(Q_{j}\right)^{\delta}<\infty\right\} .
$$

Note that in the Whitney decomposition the smaller the squares, the closer they are to the set, so to describe its geometry it is enough to work with the small squares only. Thus the large squares are dropped from the series above so that it becomes convergent.

Clearly, this definition admits the following integral reformulation (and hence does not depend on the choice of Whitney decomposition):

$$
\delta_{W h i t}(K):=\inf \left\{\delta: \int_{\Omega} \operatorname{dist}(z, K)^{\delta-2} d m(z)<\infty\right\}
$$

where $m$ denotes area, and we use the spherical metric. One can restrict integration to some neighborhood of $K$ (and should do so if working with the planar metric).

Remark 8.2 The definitions of Whitney and Poincaré exponents assume that the complement of $K=J$ is non-empty. Should $K=J$ coincide with the whole sphere, we set $\delta_{W h i t}(K)=$ $\delta_{\text {Poin }}(K):=2$.

\subsection{Multifractal analysis}

The following is Lemma 2.1 in [5], where it was used in similar situation, involving Poincaré exponent of a Kleinian group and Minkowski dimension of its limit set. We thank Chris Bishop for bringing it to our attention.

Fact 8.1 For any compact set $K, \delta_{W h i t}(K) \leq \overline{\operatorname{MDim}}(K)$. If, in addition, $K$ has zero area, then $\delta_{W h i t}(K)=\overline{\operatorname{MDim}}(K)$.

By taking a cover of $J$ by a finite number of small balls and applying Proposition 7.1 to each of them, we easily obtain the following

Lemma 8.1 Suppose that a rational function $F$ satisfies the summability condition with an exponent

$$
\alpha<\frac{2}{\mu_{\max }+2} .
$$

If the Julia set is not the whole sphere, then its Hausdorff dimension is strictly less than 2. 
It seems to be folklore that for rational maps without neutral orbits $\delta_{\text {Poin }}(J)=\delta_{W h i t}(J)$. We were unable to find a reference to this fact in the literature and thus we supply the proof below. The following is an analogue of Lemma 3.1 in [5]:

Lemma 8.2 For any rational function F without Siegel disks, Herman rings, or parabolic points one has

$$
\delta_{\text {Poin }}(J)=\delta_{W h i t}(J) .
$$

Remark 8.3 The proof below can be modified to work for parabolic points as well. However, in the presence of Siegel disks or Herman rings the introduced version of Poincaré series does not work well.

Under an additional assumption that the Julia set has zero area, the lemma together with Fact 8.1 imply that the Poincaré exponent coincides with the upper Minkowski dimension.

Proof: Fix points $\left\{z_{j}\right\}$ used in the definition of $\delta_{\text {Poin }}(J)$ - one inside each cycle of periodic Fatou components. From Lemma 7 of [18] (Lemma 11.2) follows that for any $y \in F^{-n} z_{j}$ one has

$$
\operatorname{dist}(y, J) \asymp\left|\left(F^{n}\right)^{\prime}(y)\right|^{-1},
$$

up to a constant depending on $z_{j}$ only.

Knowing that only (super) attractive Fatou components are possible, we can choose "fundamental" domains $z_{j} \in U_{j}$, so that their preimages under all possible branches of $F^{-n}$ are disjoint and cover almost all of some neighborhood $U$ of $J$ inside the Fatou set. Also $z_{j}$ and then $U_{j}$ can be chosen so that under iteration critical points never enter some neighborhoods of $U_{j}$, and hence by distortion theorems, up to a constant $\operatorname{const}\left(z_{j}, U_{j}\right)$

$$
\operatorname{dist}(x, J) \asymp\left|\left(F^{n}\right)^{\prime}(x)\right|^{-1} \asymp\left|\left(F^{n}\right)^{\prime}(y)\right|^{-1},
$$

for any $x \in F^{-n} U_{j}$ and $y$ being the corresponding preimage of $z_{j}: y \in F^{-n} z_{j}$.

Hence, for any $\delta \geq 0$ we can write (here $V \in F^{-n} U_{j}$ means that $V$ is one of the components of connectivity of the latter)

$$
\begin{aligned}
\int_{U} \operatorname{dist}(x, J)^{\delta-2} d m(x) & =\sum_{j} \sum_{n=1}^{\infty} \sum_{V \in F^{-n} U_{j}} \int_{V} \operatorname{dist}(x, J)^{\delta-2} d m(x) \\
& \asymp \sum_{j} \sum_{n=1}^{\infty} \sum_{V \in F^{-n} U_{j}} \int_{V}\left|\left(F^{n}\right)^{\prime}(x)\right|^{2-\delta}\left|\left(F^{n}\right)^{\prime}(x)\right|^{-2} d m\left(F^{n}(x)\right) \\
& \asymp \sum_{j} \sum_{n=1}^{\infty} \sum_{V \in F^{-n} U_{j}} \int_{V}\left|\left(F^{n}\right)^{\prime}\left(F^{-n} z_{j}\right)\right|^{-\delta} d m\left(F^{n}(x)\right) \\
& \asymp \sum_{j} \sum_{n=1}^{\infty} \sum_{y \in F^{-n} z_{j}}\left|\left(F^{n}\right)^{\prime}(y)\right|^{-\delta} \int_{U_{j}} d m(z) \asymp \Sigma_{\delta}\left(J,\left\{z_{j}\right\}\right),
\end{aligned}
$$

which clearly implies the desired equality. 
The definitions and our discussion so far imply two chains of inequalities:

$$
\operatorname{HDim}(J) \leq \underline{\operatorname{MDim}}(J) \leq \overline{\operatorname{MDim}}(J)
$$

and

$$
\delta_{\text {Poin }}(J)=\delta_{W h i t}(J) \leq \overline{\operatorname{MDim}}(K) .
$$

Proposition 8.1 (Gauge function estimate for conformal measure) Suppose that a rational function $F$ satisfies the summability condition with an exponent

$$
\alpha<\frac{q}{\mu_{\max }+q}
$$

and $\nu$ is q-conformal measure with no atoms at critical points. Then for $\nu$-almost every $x \in J$ and any $\epsilon>0$ there are constants $C_{x}>0$ and $C_{x, \epsilon}>0$, such that for any ball $B(x, r), r<1$, centered at $x$ one has

$$
C_{x} r^{q}<\nu(B(x, r))<C_{x, \epsilon} r^{q-\epsilon}
$$

Proof: It is a straight-forward use of Proposition 5.2.

It is easy to see, that the proposition above implies that $\operatorname{HDim}(J) \geq \operatorname{HDim}(\nu)=q$. Combining this with the Corollary 5.1, we obtain the following

Corollary 8.1 Assume that $F$ satisfies the summability condition with an exponent

$$
\alpha<\frac{\delta_{\text {Poin }}(J)}{\mu_{\max }+\delta_{\text {Poin }}(J)},
$$

then

$$
\operatorname{HDim}(J) \geq \delta_{\text {Poin }}(J)=\operatorname{HDim}(\nu)
$$

Proof of Theorem 7. By now we have

$$
\delta_{\text {Poin }}(J)=\delta_{\text {conf }}(J)=\delta_{W h i t}(J)=\operatorname{MDim}(J) \geq \operatorname{HDim}(J) \geq \delta_{\text {Poin }}(J),
$$

and hence all these dimensions coincide, provided that a rational function $F$ satisfies the summability condition with an exponent

$$
\alpha<\frac{\delta_{\text {Poin }}(J)}{\mu_{\max }+\delta_{\text {Poin }}(J)} .
$$

By the work of M. Denker, F. Przytycki, and M. Urbanski, the hyperbolic and dynamical dimensions will also be equal to the dimensions above. 


\section{Removability and rigidity}

In this Section, we prove Theorems 8, 9, and 10.

\subsection{Conformal removability and strong rigidity}

The notion of conformal removability (also called holomorphic removability) appears naturally in holomorphic dynamics: often one can show that two dynamical systems are conjugated by a homeomorphism which is (quasi)conformal outside the Julia set, and conformal removability of the latter ensures global (quasi)conformality of the conjugation.

Definition 9.1 We say that a compact set $J$ is conformally removable if any homeomorphism of the Riemann sphere $\hat{\mathbb{C}}$, which is conformal outside $K$, is globally conformal and hence is a Möbius transformation.

The quasiconformal removability is defined similarly. An easy application of the measurable Riemann mapping theorem shows that the two notions are equivalent. The problem of geometric characterization of removable sets is open, see [24] for discussion and relevant references. Sets of positive area are non-removable, as are Cartesian products of intervals with Cantor sets of positive length. On the other hand, quasicircles and sets of $\sigma$-finite length are removable. Note that there are removable sets of Hausdorff dimension 2 and non-removable of dimension 1.

In [24] a few geometric criteria for removability are given, some close to being optimal and well-adapted for dynamical applications. We will use the following fact (which is Theorem 5 in $[24])$ :

Fact 9.1 Suppose that $F$ is a polynomial, and $\left\{B_{j}\right\}$ is a finite collection of domains whose closure covers $J_{F}$. Denote by $\left\{P_{i}^{n}\right\}$ the collection of all components of connectivity of pullbacks $F^{-n} B_{j}$, and by $N\left(P_{i}^{n}\right)$ the degree of $F^{n}$ restricted to $P_{i}^{n}$. Then the geometric condition,

$$
\sum_{i, n} N\left(P_{i}^{n}\right) \operatorname{diam}\left(P_{i}^{n}\right)^{2}<\infty
$$

is sufficient for conformal removability of the Julia set.

If a polynomial satisfies the summability condition with an exponent $\alpha<\frac{2}{\mu_{\max }+2}$ then Proposition 7.1 implies condition (26) for a cover by sufficiently small balls $B_{j}$. By Fact 9.1 , the Julia set is conformally removable and Theorem 8 follows. Similar reasoning works for every Julia set, which is a boundary of one of the Fatou components.

\subsection{Dynamical removability and rigidity}

The assumption that the Julia set coincides with the boundary of one of the Fatou components is essential for conformal removability. Indeed, there are hyperbolic rational functions with nonremovable Julia sets. An example of a non-removable Julia set, which is topologically a Cantor set of circles, is constructed in $\S 11.8$ of the book [2]. It is a classical observation that these type 
of sets are not conformally removable. An exotic homeomorphism is given by rotating annuli between circles by a devil's staircase of angles: the resulting homeomorphism is conformal on each annulus, globally continuous since the devil's staircase is, but clearly is not Möbius (i.e. not globally conformal).

Even though such Julia sets are not conformally removable, they will be removable for all "dynamical" conjugacies. To make this precise, consider a homeomorphism $\phi$ which conjugates a rational dynamical system $(\hat{\mathbb{C}}, F)$ to another dynamical system $(\hat{\mathbb{C}}, G)$ and assume that $\phi$ is quasiconformal outside the Julia set $J$.

Recall a metric definition of quasiconformality (which states that images of circles look like circles themselves): a homeomorphism $\phi$ is quasiconformal, if there is a constant $H$ such that for every point $x \in \hat{\mathbb{C}}$

$$
\limsup _{r \rightarrow 0} \frac{L_{\phi}(x, r)}{l_{\phi}(x, r)} \leq H<\infty
$$

where

$$
\begin{aligned}
L_{\phi}(x, r) & :=\sup \{|\phi(x)-\phi(y)|:|x-y| \leq r\} \\
l_{\phi}(x, r) & :=\inf \{|\phi(x)-\phi(y)|:|x-y| \geq r\} .
\end{aligned}
$$

If a rational function $F$ is hyperbolic, then every sufficiently small ball with center at the Julia set is mapped univalently by some iterate of $F$ to a large scale with bounded distortion, and the inequality (27) holds by a compactness argument implying a (global) quasiconformality of $\phi$.

For non-hyperbolic maps the property of "going to large scale with bounded distortion" fails for many small balls. In these circumstances one has to resort to more subtle tools in the theory of quasiconformal maps. A theorem of great use for complex dynamical systems was proved recently by J. Heinonen and P. Koskela in [22]. They have shown, that for Euclidean spaces the upper limit "limsup" in the metric definition of the quasiconformality can be replaced by "lim inf." J. Heinonen and P. Koskela's result was immediately applied by F. Przytycki and S. Rohde [39] to deduce rigidity of Julia sets satisfying the topological Collet-Eckmann condition (shortly TCE). The argument of [39] goes as follows: for every point $x \in J$ there is a sequence of radii $r_{j} \rightarrow 0$ such that the balls $B_{r_{j}}(x)$ are mapped by some iterates of $F$ to a large scale with bounded distortion (though no longer univalently but with uniformly bounded criticality), and the inequality (27) for lim inf holds again by a compactness argument implying a (global) quasiconformality of $\phi$.

Rational maps which satisfy the summability condition have weaker properties than TCE maps (in the unicritical case the latter class is strictly smaller), so we need an even stronger theorem than that of J. Heinonen and P. Koskela. It is a well-known fact, that the metric definition (with "lim sup") of quasiconformality allows for an exceptional set. Partially motivated by the perspective applications to our paper, S. Kallunki and P. Koskela [25] established very recently that one can also have an exceptional set in the "lim inf" definition of quasiconformality. The following is Theorem 1 of [25]: 
Fact 9.2 Let $\Omega \subset \mathbb{R}^{n}$ be a domain and suppose that $\phi: \Omega \rightarrow \phi(\Omega) \subset \mathbb{R}^{n}$ is a homeomorphism. If there is a set $E$ of $\sigma$-finite $(n-1)$ - dimensional Hausdorff measure so that

$$
\liminf _{r \rightarrow 0} \frac{L_{\phi}(x, r)}{l_{\phi}(x, r)} \leq H<\infty
$$

for each $x \in \Omega \backslash E$, then $\phi$ is quasiconformal in $\Omega$.

This theorem fits very well into our framework. By Proposition 5.1, if $F$ satisfies the summability condition with an exponent $\alpha<\frac{1}{\mu_{\max }+1}$, then except for a set $E$ of Hausdorff dimension $<1$ every point $x \in J$ "go to a large scale" infinitely often. More precisely, for every $x \in J$ there exists (a point-dependent) sequence of radii $r_{j} \rightarrow 0$ such that the balls $B_{r_{j}}(x)$ are mapped by iterates $F^{k_{j}}$ to the large scale of size $\asymp R^{\prime}$ univalently and with a bounded distortion. Thus for every $x \in J \backslash E$ (cf. [39]) one has

$$
\begin{aligned}
\liminf _{r \rightarrow 0} \frac{L_{\phi}(x, r)}{l_{\phi}(x, r)} & \leq \liminf _{j \rightarrow \infty} \frac{L_{\phi}\left(x, r_{j}\right)}{l_{\phi}\left(x, r_{j}\right)} \lesssim \liminf _{j \rightarrow \infty} \frac{L_{\phi}\left(F^{n_{j}}(x), R^{\prime}\right)}{l_{\phi}\left(F^{n_{j}}(x), R^{\prime}\right)} \\
& \lesssim \sup _{x \in J} \frac{L_{\phi}\left(x, R^{\prime}\right)}{l_{\phi}\left(x, R^{\prime}\right)}=: H<\infty,
\end{aligned}
$$

the latter quantity being finite by a compactness argument. We infer that the homeomorphism $\phi$ is globally quasiconformal, thus deducing Theorem 9 .

Consider now a quasiconformal homeomorphism $\phi$ which conjugates a rational dynamical system $(\hat{\mathbb{C}}, F)$ to another dynamical system $(\hat{\mathbb{C}}, G)$ and assume that $F$ satisfies the (weaker) summability condition with an exponent $\alpha<\frac{2}{\mu_{\max }+2}$.

If $J \neq \hat{\mathbb{C}}$ then the area of the Julia set is zero, by the Corollary 1.2 , and an invariant Beltrami coefficient $\phi_{\mu}$ has to be supported on the Fatou set. There are two interesting special settings when $\phi$ is automatically a Möbius transformation. If $\phi$ is conformal outside the Julia set, then the Beltrami coefficient is identically zero, and $\phi$ is Möbius. Also, if there is only one simply-connected Fatou component (e.g. this is the case for polynomials with all critical points in the Julia set), it has to be super-attracting, and by a standard argument it does not support non-zero $F$-invariant Beltrami coefficients, and $\phi$ is Möbius again.

If $J=\hat{\mathbb{C}}$ then by Proposition 5.1 except for a set $E$ of Hausdorff Dimension $<2$, all points "go to a large scale" infinitely often. Then a standard technique (see the proof of either Theorem 3.9 or Theorem 3.17 in [32]) implies that the Beltrami coefficient $\mu_{\phi}$ has to be holomorphic, and by Lemma 3.16 from [32], we have that $\mu_{\phi} \equiv 0$ or $F$ is a double cover of an integral torus endomorphism, i.e. it is a Lattés example. This concludes the proof of Theorem 10.

\section{Integrability condition and invariant measures.}

A natural question arises whether a rational map $F$ has invariant measures absolutely continuous with respect to conformal measures. We will make two different types of assumptions. Firstly, we make a general assumption about regularity of conformal measures. This will guarantee that a conformal measure is not too singular with respect to the corresponding Hausdorff 
measure (integrability condition). Secondly, we demand that $F$ has some expansion property, usually given in the form of a suitable summability condition.

Let $\nu$ be a conformal measure with an exponent $\delta$ defined on the Julia set $J$. Assume also that $\nu$ satisfies the uniform integrability condition with an exponent $\eta$. We recall that this means that there exist positive $C$ and $\eta$ such that for all positive integers $n$ and every $c \in$ Crit,

$$
\int \frac{d \nu}{\operatorname{dist}\left(x, F^{n}(c)\right)^{\eta}}<C<\infty
$$

\subsection{Ruelle-Perron-Frobenius transfer operator.}

We study the existence of an absolutely continuous invariant measure $\sigma$ through analysis of the Ruelle-Perron-Frobenius operator $\mathcal{L}$ which ascribes to every measure $\nu$, the density of $F_{*}(\nu)$ with respect to $\nu$. The $N$-th iterate of $\mathcal{L}(\nu)$ evaluated at $z$ is equal to

$$
\mathcal{L}^{N}(\nu)(z):=\frac{d F_{*}^{N}(\nu)}{d \nu}=\sum_{y \in F^{-N}(z)} \frac{1}{\left|\left(F^{N}\right)^{\prime}(y)\right|^{\delta}} .
$$

For simplicity, we will drop $\nu$ from the notation of the Ruelle-Perron-Frobenius operator.

Proposition 10.1 Assume that a rational function $F$ satisfies the summability condition with an exponent

$$
\alpha<\frac{\delta}{\delta+\mu_{\max }}
$$

and $\nu$ is a $\delta$-conformal measure on $J$. Let $\Delta_{k}:=\operatorname{dist}\left(f^{k}(\right.$ Crit $\left.), z\right)$ and

$$
\mathrm{g}(z):=\sum_{k=1}^{\infty} \gamma_{k}^{-\delta} \Delta_{k}^{-\left(1-\frac{1}{\mu_{\max }}\right) \delta} .
$$

There exists a positive constant $K$ such that for every $z \notin \bigcup_{n=1}^{\infty} F^{n}$ (Crit) and every positive integer $N$,

$$
\mathcal{L}^{N}(z)<K \mathrm{~g}(z)
$$

The sequence $\gamma_{k}^{-1}$ (defined in Lemma 2.2) is summable with an exponent $\beta=\frac{\mu_{\max } \alpha}{1-\alpha}<\delta$.

Proof: We will use the estimates of the Main Lemma for a modified decomposition procedure, as in Subsection 5.1.

Construction. Let $z \in X$ and a sequence

$$
F^{-N}(z), \ldots, F^{-1}(z), z
$$

form a chain of preimages, that is $F\left(F^{-i}(z)\right)=F^{-i+1}(z)$ for $i=1, \ldots, N$ and $F^{-N}(z) \in X$. We decompose the chain into blocks of preimages of the types 2 and $1 \ldots 13$. This is done using 
the procedure of the Main Lemma with the following stopping rule: at the first occurrence of a type 2 block we stop the procedure of decomposing the chain, so this type 2 block can be arbitrarily long. See Subsection 5.1 for the details.

The inductive procedure gives a coding of backward orbits by sequences of 1's, 2's and 3's, with only the following three types of codings allowable: $2,1 \ldots 3,21 \ldots 13$. We recall that according to our convention, during the inductive procedure we put symbols in the coding from the right to the left.

We attach to every chain of preimages of $z$ the sequence $k_{l}, \ldots, k_{0}$ of the lengths of the blocks of preimages of a given type in its coding. Again our convention requires that $k_{0}$ always stands for the length of the rightmost block of preimages in a coding. Clearly, $k_{0}+\cdots+k_{l}=N$.

Estimates. We recall that the Main Lemma implies that every sequence of the form $11 \ldots 3$ with the length of the corresponding pieces $k_{l}, \ldots k_{0}$ yields expansion

$$
\gamma_{k_{l}} \cdot \ldots \cdot \gamma_{k_{0}} \Delta_{k_{0}}^{1-\frac{1}{\mu_{\max }}}
$$

where $\Delta_{k_{0}}=\operatorname{dist}\left(f^{k_{0}}(\right.$ Crit $\left.), z\right)$.

For singleton sequences $\{2\}$ of the length $n$ we have the following estimate,

$$
\sum_{y \in F^{-n}(x)} \frac{1}{\left|\left(F^{n}\right)^{\prime}(y)\right|^{\delta}} \lesssim \sum_{F^{-n}} \frac{\nu\left(F^{-n}\left(B_{R^{\prime}}(x)\right)\right)}{\nu\left(B_{R^{\prime}}(x)\right)} \leq \frac{1}{\nu\left(B_{R^{\prime}}(x)\right)} \leq K_{R^{\prime}},
$$

which is independent from $n$.

Consider now a set of all preimages $y \in F^{-N}(z)$. Let $11 \ldots 13$ denote the set of all points $x \in \bigcup_{i=1}^{N} F^{-i}(z)$ which are coded by maximal sequences of 1's and 3's. We define $n_{x}$ by the condition $F^{n_{x}}(x)=z$.

Hence,

$$
\begin{aligned}
\mathcal{L}^{N}(z) & =\sum_{\text {all codings }} \frac{1}{\left|\left(F^{N}\right)^{\prime}(y)\right|^{\delta}} \\
& \leq \sum_{n_{x}=1}^{N}\left(\sum_{11 \ldots 13} \frac{1}{\left|\left(F^{n_{x}}\right)^{\prime}(x)\right|^{\delta}}\right)\left(\sum_{y \in F^{-N+n_{x}}} \frac{1}{\left|\left(F^{n}\right)^{\prime}(y)\right|^{\delta}}\right) \\
& \leq K_{R^{\prime}} \sum_{11 \ldots 13}\left(\gamma_{k_{l}} \cdot \ldots \cdot \gamma_{k_{0}}\right)^{-\delta} \Delta_{k_{0}}^{-\left(1-\frac{1}{\mu_{\max }}\right) \delta}
\end{aligned}
$$

Since for every sequence of $k_{l}, \ldots, k_{0}$ positive integers there is at most $(2 \operatorname{deg} F)^{l+1}$ sequences $11 \ldots 13$ with the corresponding lengths of the pieces of type 1 and 3 (see the estimate (17)), we obtain that

$$
\mathcal{L}^{N}(z) \lesssim \sum_{l, k_{l}, \ldots, k_{0}}(4 \operatorname{deg} F)^{l+1}\left(\gamma_{k_{l}} \cdot \ldots \cdot \gamma_{k_{0}}^{-\delta} \Delta_{k_{0}}^{-\left(1-\frac{1}{\mu_{\max }}\right) \delta}\right.
$$




$$
\begin{aligned}
& \left.<\sum_{l=1}^{\infty}\left(4 \operatorname{deg} F \sum_{k_{l}} \gamma_{k_{l}}^{-\delta}\right) \cdot \ldots \cdot\left(4 \operatorname{deg} F \sum_{k_{1}} \gamma_{k_{1}}^{-\delta}\right) \cdot\left(4 \operatorname{deg} F \sum_{k_{0}} \gamma_{k_{0}}\right)^{-\delta} \Delta_{k_{0}}^{-\left(1-\frac{1}{\mu_{\max }}\right) \delta}\right) \\
& <\sum_{l=1}\left(\frac{1}{4}\right)^{l}\left(4 \operatorname{deg} F \sum_{k_{0}} \gamma_{k_{0}}^{-\delta} \Delta_{k_{0}}^{-\left(1-\frac{1}{\mu_{\max }}\right) \delta}\right) \\
& <K \sum_{k_{0}}\left(\gamma_{k_{0}}\right)^{-\delta} \Delta_{k_{0}}^{-\left(1-\frac{1}{\mu_{\max }}\right) \delta} .
\end{aligned}
$$

By Lemma 2.2, $\gamma_{k}$ is summable with any exponent bigger than $-\frac{\mu_{\max } \alpha}{1-\alpha}$.

Proof of Theorem 5. Now it is a standard reasoning. By Proposition 10.1, $\mathcal{L}^{n}(z) \leq \mathrm{g}(z)$ and $\mathrm{g}(z) \in L^{1}(\nu)$. Hence,

$$
\nu_{N}=\frac{1}{N} \sum_{i=0}^{N-1} F_{*}^{i}(\nu)
$$

form a weakly compact set of probabilistic measures absolutely continuous with respect to $\nu$ with densities bounded by g. A weak limit of $\nu_{N}$ gives an absolutely continuous invariant measure.

Uniqueness and ergodicity follow by an argument presented in Section 6.2.

\subsection{Regularity of invariant densities}

Corollary 10.1 Suppose that F satisfies the summability condition with an exponent

$$
\alpha<\frac{\delta}{\delta+\mu_{\max }} .
$$

Let $\eta_{1}>\eta_{0}:=\delta\left(1-\frac{1}{\mu_{\max }}\right)$ and assume $\eta$-integrability of the unique $\delta_{\text {Point-conformal measure }}$ $\nu$ for every $\eta \in\left(0, \eta_{1}\right)$. Then for every $\zeta<\eta_{1} / \eta_{0}$

$$
\int\left(\frac{d \sigma}{d \nu}\right)^{\zeta} d \nu<\infty
$$

and for every Borel set A,

$$
\sigma(A) \leq K_{\zeta} \nu(A)^{1-1 / \zeta}
$$

Proof: To prove $\zeta$-integrability of the density of $d \sigma / d \nu$ we use Proposition 10.1. Recall that $\Delta_{k}=\operatorname{dist}\left(f^{k}(\mathrm{Crit}), z\right)$. We use the Hölder inequality with the exponents $1 / \zeta+1 / \zeta^{\prime}=1$ in the estimates below.

$$
\sum_{k} \gamma_{k}^{-\delta} \Delta_{k}^{-\left(1-1 / \mu_{\max }\right) \delta}=\sum_{k} \gamma_{k}^{-\delta / \zeta^{\prime}} \frac{\gamma_{k}^{-\delta / \zeta}}{\Delta_{k}^{\left(1-1 / \mu_{\max }\right) \delta}}
$$




$$
\begin{aligned}
& \leq\left(\sum_{k} \gamma_{k}^{-\delta}\right)^{1 / \zeta^{\prime}}\left(\sum_{k} \frac{\gamma_{k}^{-\delta}}{\Delta_{k}^{\delta\left(1-1 / \mu_{\max }\right) \zeta}}\right)^{1 / \zeta} \\
& \leq K\left(\sum_{k} \frac{\gamma_{k}^{-\delta}}{\Delta_{k}^{\eta}}\right)^{1 / \zeta}
\end{aligned}
$$

with $\eta \in\left[0, \eta_{1}\right)$. Next, we integrate the density $d \sigma / d \nu$ to the power $\zeta$. Proposition 10.1 and the inequality (30) imply that

$$
\begin{aligned}
\int\left(\frac{d \sigma}{d \nu}\right)^{\zeta} d \nu & \lesssim \int\left(\sum_{k} \gamma_{k}^{-\delta} \Delta_{k}^{-\left(1-1 / \mu_{\max }\right) \delta}\right)^{\zeta} d \nu \\
& \lesssim \sum_{k} \gamma_{k}^{-\delta} \int \Delta_{k}^{-\eta} d \nu<\infty
\end{aligned}
$$

To prove the last estimate of the Corollary, we apply once more the Hölder inequality with the exponents $\zeta$ and $\zeta^{\prime}$.

$$
\begin{aligned}
\sigma(A) & =\int_{A} \frac{d \sigma}{d \nu} d \nu \leq\left(\int_{A} d \nu\right)^{1 / \zeta^{\prime}}\left(\int_{A}\left(\frac{d \sigma}{d \nu}\right)^{\zeta} d \nu\right)^{1 / \zeta^{\prime}} \\
& \leq K_{\zeta} \nu(A)^{1-1 / \zeta}
\end{aligned}
$$

Proposition 10.2 Under the assumptions of Theorem 5, there exists a positive constant $C$ so that for $\nu$ almost every point $z$,

$$
\frac{d \sigma}{d \nu}(z) \geq C
$$

where $d \sigma / d \nu$ stands for the density of the absolutely continuous invariant measure $\sigma$.

Proof: Let $\mathrm{g}=\sum_{k=1}^{\infty} \gamma_{k}^{-\delta} \Delta_{k}^{-\left(1-\frac{1}{\mu_{\max }}\right) \delta}$ and $\mathrm{g}_{n}(z):=\sum_{k=n}^{\infty} \gamma_{k}^{-\delta} \Delta_{k}^{-\left(1-\frac{1}{\mu_{\max }}\right) \delta}$. By the hypothesis of Theorem 5 (the intergrability condition),

$$
\int \mathrm{g}(z) d \nu<\infty
$$

and thus for almost all $z \in \hat{\mathbb{C}}$ with respect to $\nu, \lim _{n \rightarrow \infty} \mathrm{g}_{n}(z)=0$. Consequently, there exist a point $w \notin \bigcup_{n=1}^{\infty} F^{-n}$ (Crit) and a positive integer $N$ so that $d \sigma / d \nu(w) \geq 1$ and $\mathrm{g}_{n}(w)<1 / 2$ for every $n \geq N$. Observe that the choice of $N$ and $w$ does not depend on $R^{\prime}$.

Choose $R^{\prime}$ so small that $B_{R^{\prime}}(w) \cap F^{k}$ (Crit) $=\emptyset$ for all $k \leq N$ and decompose the backward orbit of $w$ into pieces of type 1,2 or 3 as described in the proof of Proposition 10.1. By the construction, only the following three types of codings are allowable: $2,1 \ldots 3,21 \ldots 3$.

Let $\mathcal{R}_{R^{\prime}}^{n}(z)=\sum_{\{2\}}\left|\left(F^{n}\right)^{\prime}(y)\right|^{-\delta}$ be the sum over all type 2 preimages of $z$ of length $n$ (regular part). The sum over all other preimages is denoted by $\mathcal{S}_{R^{\prime}}^{n}(z)$ (singular part, compare [37]). By 
the choice of $R^{\prime}, \mathcal{S}_{R^{\prime}}^{n}(w)<1 / 2$ for every $n$ positive. Hence, by the choice of $w$ and for $n$ large enough

$$
\frac{3}{4} \leq \frac{1}{n} \sum_{i=0}^{n-1} \mathcal{L}^{i}(w)=\frac{1}{n} \sum_{i=0}^{n-1} \mathcal{R}_{R^{\prime}}^{i}(w)+\frac{1}{n} \sum_{i=0}^{n-1} \mathcal{S}_{R^{\prime}}^{i}(w) \leq \frac{1}{n} \sum_{i=0}^{n-1} \mathcal{R}_{R^{\prime}}^{i}(w)+\frac{1}{2} .
$$

We choose new $R_{\text {new }}^{\prime}:=R^{\prime} / 2$. This will change the decomposition of backward orbits of points, generally allowing more type 2 sequences of preimages. If $z \in B_{R^{\prime} / 2}(w)$ then every type 2 preimage of $w$ with the parameter $R^{\prime}$ corresponds to exactly one type 2 preimage of $z$ with $R_{\text {new }}^{\prime}$. By the bounded distortion, $\mathcal{R}_{R^{\prime} / 2}^{n}(z) \gtrsim \mathcal{R}_{R^{\prime}}^{n}(w)$ and thus for $n$ large enough,

$$
\frac{1}{n} \sum_{i=0}^{n-1} \mathcal{L}^{i}(z) \geq \frac{1}{n} \sum_{i=0}^{n-1} \mathcal{R}_{R^{\prime}}^{i}(w) \gtrsim 1 / 4
$$

By the eventually onto property, there exists a positive integer $m$ so that for every $z \in J$ there is a preimage $u=F^{-m}(z) \in B_{R^{\prime}}(w)$. Let $M=\sup _{z \in J}\left|F^{\prime}(z)\right|$. Then for almost every $z \in \hat{\mathbb{C}}$ with respect to $\nu$,

$$
d \sigma / d \nu(z)=\lim _{n \rightarrow \infty} \frac{1}{n} \sum_{i=0}^{n-1} \mathcal{L}^{i}(z) \geq M^{-m} \lim _{n \rightarrow \infty} \frac{1}{n} \sum_{i=j}^{n-1} \mathcal{L}^{i-j}(u) \gtrsim M^{-m} / 2 .
$$

\subsection{Analytic maps of interval}

We are interested in analytic maps $f$ with negative Schwarzian derivative (i.e. $S(f)<0$, recall the formula (3)), which map a compact interval $I$ with non-empty interior into itself. We will denote by $F$ a complex extension of $f$ to a neighborhood $U_{F} \supset I$ in the complex plane which does not contain any critical points different then the real ones. We will study iterates of real inverse branches $F_{\mathrm{rl}}^{-1}$ of $F$ defined by the condition: if $U \subset U_{F}$ is a topological disk such that $U \cap \mathbb{R}$ is an interval then for every $x \in U \cap \mathbb{R}, F_{\mathrm{rl}}^{-1}(x) \in I$. By the definition, $F_{\mathrm{rl}}^{-1}(U)$ is a topological disk and $F_{\mathrm{rl}}^{-1}(U \cap \mathbb{R})=F_{\mathrm{rl}}^{-1}(U) \cap \mathbb{R}$.

We will show that iterates of points by the real inverse branches stay in a bounded distance from the interval $I$. To this aim we will need the disk property based on Proposition 3 and Proposition 4 of [17]. For every $a, b \in \mathbb{R}$ define $D_{a, b}$ to be the open disk centered at $(a+b) / 2$ with the radius $|a-b| / 2$.

Fact 10.1 Let $h: I \rightarrow \mathbb{R}$ be an analytic diffeomorphism from a compact interval $I$ with nonempty interior into the real line. Suppose that $f: I \rightarrow \mathbb{R}$ either concides with $h$ or is of the form $h^{\ell}, \ell>1$ is an integer. In the latter case assume that there exists $\zeta \in I$ such that $h(\zeta)=0$. Let $H$ be an extension of $h$ to a complex neighborhood of $I$ and $F(z)$ be equal either to $H(z)$ or $H(z)^{\ell}$. If $S(f)<0$ on I then there exists $\delta>0$ such that for every two distinct points $a, b \in I$ with $\zeta \notin(a, b)$ and diam $D_{f(a), f(b)}<\delta$ the connected component of $F^{-1}\left(D_{f(a), f(b)}\right)$ which contains $(a, b)$ is contained in $D_{a, b}$. 
Lemma 10.1 There exists a constant $L>0$ so that for every $n \in \mathbb{N}$, every $x \in I$, and every inverse branch $f^{-n}$ defined on $B_{L}(x) \cap \mathbb{R}$, the real inverse branch $F_{\mathrm{rl}}^{-n}$ which coincides with $f^{-n}$ on $B_{L}(x) \cap \mathbb{R}$ is well defined on $B_{L}(x)$ and $F_{\mathrm{rl}}^{-n}\left(B_{L}(x)\right) \subset U_{F}$.

Proof: Observe that $f$ has only finitely many critical points in $I$ and for every critical point $c \in$ Crit there exists a neighborhood $U_{c} \ni c$ such that $F$ is in the form $F(c)\left(1-H_{c}(z)^{\ell(c)}\right)$ with $H_{c}$ a biholomorphic function near $c$. The proof follows immediately from Fact 10.1 .

Proof of Theorem 6. The normalized Lebesgue measure $\nu$ on $I$ can be regarded as a $\delta$ conformal measure with an exponent $\delta=1$. Clearly, $\nu$ satisfies the uniform integrability condition for any $\eta<1$. We will show that we can use the estimates of Proposition 10.1 for the real inverse branches of $F$.

From Lemma 10.1 we infer that all preimages of disks $B_{L}(x)$ by the real inverse branches of $F$ are well-defined and of uniformly bounded diameters. This means that the estimates of Lemma 3.2 (the Main Lemma) for disks $B_{\Delta}(x), x \in \mathbb{R}$, and the real inverse branches $F_{\mathrm{rl}}^{-N}$ are still valid.

We define a real part $\mathcal{L}_{\text {rl }}$ of the Ruelle-Perron-Frobenius operator $\mathcal{L}$ by,

$$
\mathcal{L}_{\mathrm{rl}}(\nu)(z):=\sum_{y \in F^{-1}(z) \cap \mathbb{R}} \frac{1}{\left|F^{\prime}(y)\right|^{\delta}} .
$$

In the proof of Proposition 10.1, the equality $\operatorname{Jac}_{\nu}(x)=\left|F^{\prime}(x)\right|^{\delta}$ was used only for the estimates involving type 2 preimages. Explicitly, it is the estimate (29). Let $F_{\mathrm{rl}}^{-k}$ be a real inverse branch of type 2 (we use the inductive procedure from the proof of Proposition 10.1) and $y=F_{\mathrm{rl}}^{-k}(x)$. We have the following uniform estimate,

$$
\left|\left(F^{k}\right)^{\prime}(y)\right| \sim \frac{\nu\left(B_{R^{\prime}}(x)\right)}{\nu\left(F^{-k}\left(B_{R^{\prime}}(x)\right)\right)} .
$$

The inductive construction of Proposition 10.1 yields a coding of backward orbits by sequences of 1's, 2's, and 3's. We recall that only three types of the codings are allowable: 2, $1 \ldots 13,21 \ldots 13$. Lemma 10.1 guarantees that only the critical points of $f$ are used in the inductive construction of the backward codings.

After these preparations we are ready to invoke Proposition 10.1 with $\mathcal{L}$ replaced by $\mathcal{L}_{\mathrm{rl}}$. Hence, there exists a positive constant $K$ such that for every $z \in \mathbb{R} \backslash \bigcup_{n=1}^{\infty} f^{n}$ (Crit) and every positive integer $N$,

$$
\mathcal{L}_{\mathrm{rl}}^{N}(\nu)(z)=\sum_{y \in F^{-N}(z) \cap \mathbb{R}} \frac{1}{\left|\left(F^{N}\right)^{\prime}(y)\right|^{\delta}}<K \sum_{k=1}^{\infty} \gamma_{k}^{-\delta} \Delta_{k}^{-\left(1-\frac{1}{\mu \max }\right) \delta},
$$

where $\Delta_{k}=\operatorname{dist}\left(f^{k}(\mathrm{Crit}), z\right)$ and the sequence $\gamma_{k}^{-1}$ (defined in Lemma 2.2) is summable with an exponent smaller than $\delta$. To complete the proof, observe that $\mathcal{L}_{\text {rl }}$ with $\delta=1$ is the 
Ruelle-Perron-Frobenius operator for $f$. Therefore, the densities of $\nu_{N}=f_{*}^{N}(\nu)$ are bounded by a constant multiple of an $L^{1}$ function $\sum_{k=1}^{\infty} \gamma_{k}^{-1} \Delta_{k}^{-\left(1-\frac{1}{\mu \max }\right)}$. Any weak limit of $\nu_{N}$ gives an absolutely continuous invariant measure for $f$. The additional claims of Theorem 6 follow immediately from Corollary 10.1.

\section{Geometry of Fatou components}

\subsection{Integrable domains}

Lemma 11.1 For every periodic Fatou component $\mathcal{F}$ the following two conditions are equivalent:

1. For any (some - by the Koebe distortion lemma 2.1 the statements are equivalent) point $z \in \mathcal{F}$ away from the critical orbits there exist a sequence $\omega_{n}$ so that $\left|\left(F^{n}\right)^{\prime}(y)\right|>\omega_{n}$ for points $y \in \mathcal{F} \cap F^{-n} z$, and $\sum_{n=1}^{\infty} \omega_{n}^{-1}<\infty$.

2. $\mathcal{F}$ is an integrable domain.

Without loss of generality we may assume that $F$ fixes a Fatou component $\mathcal{F}$. Throughout the rest of this Section we will always mean by $F^{-n}$ a branch mapping $\mathcal{F}$ to itself.

Take a subdomain $\Omega \subset \mathcal{F}$ with smooth boundary containing all critical points from $\mathcal{F}$ such that $F \Omega \subset \Omega$. Any point $z \in \mathcal{F}$ eventually gets to $\Omega$ under some iterate of $F$, so we can define $n(z):=\min \left\{n: F^{n}(z) \in \Omega\right\}$. Also fix a reference point $z_{0} \in \Omega$.

Lemma 11.1 follows immediately from Lemma 7 from [18] which we state as Lemma 11.2.

Lemma 11.2 Suppose that $z \notin \Omega$ and $n=n(z)$. Then

$$
\begin{aligned}
\operatorname{dist}(z, \partial \mathcal{F}) & \asymp\left|\left(F^{n}\right)^{\prime}(z)\right|^{-1}, \\
\operatorname{dist}_{\mathrm{qh}}\left(z, z_{0}\right) & \asymp n,
\end{aligned}
$$

up to some constant depending on $\mathcal{F}$ and our choice of $\Omega$ only.

The proof of Theorem 11 is preceded by a few analytical observations, compare [16] and [43]. We will use that quasihyperbolic metric is a geodesic metric, see exposition [27] for this and other properties of quasihyperbolic metric.

Lemma 11.3 Every integrable domain (see Definition 1.7) is geodesically bounded, i.e. every minimal quasihyperbolic geodesic is of uniformly bounded Euclidean arclength.

Proof: Let $\gamma$ be a (minimal) quasihyperbolic geodesic joining $z_{0}$ and $z$, i.e. $\operatorname{dist}_{\mathrm{qh}}\left(z_{0}, z\right)=$ length $_{\mathrm{qh}}(\gamma)$. We parameterize $\gamma$ by its Euclidean arclength starting from $z_{0}$. We define function $g$ on the interval $[0$, length $(\gamma)]$ by $g(t):=\operatorname{length}_{\mathrm{qh}}(\gamma[0, t])=\operatorname{dist}_{\mathrm{qh}}\left(z_{0}, \gamma(t)\right)$, the latter quantities are identical since the geodesic is minimal. Then

$$
\frac{d}{d t} g(t)=\operatorname{dist}(\gamma(t), \partial \mathcal{F})^{-1}
$$


From the definition of integrable domains, we obtain the following differential inequality

$$
\frac{d}{d t} g(t) \cdot H(g(t)) \geq 1
$$

Integrating leads to

$$
s=\int_{0}^{s} d t \leq \int_{0}^{s} H(g(t)) d g(t) \leq \int_{0}^{\infty} H(r) d r<\infty
$$

Denote by $\gamma(z, y)$ a minimal quasihyperbolic geodesic joining $z, y \in \Omega$, that is $\operatorname{dist}_{\mathrm{qh}}(z, y)=$ length $_{\mathrm{qh}}(\gamma(z, y))$.

Lemma 11.4 Let $z_{0}$ be a base point in $\Omega$ (see Definition 1.7). There exists a continuous decreasing function $A: \mathbb{R}_{+} \rightarrow \mathbb{R}_{+}$with $\lim _{r \rightarrow 0} A(r)=\infty$ so that for every point $z_{1} \in \gamma$

$$
\operatorname{dist}_{\mathrm{qh}}\left(z_{0}, z\right) \leq A\left(\text { length }\left(\gamma\left(z_{1}, z\right)\right)\right) .
$$

Proof: We follow the notation from the proof of Lemma 11.3. Let length $\mathrm{qh}_{\mathrm{h}}\left(\gamma\left(z_{0}, z_{1}\right)\right)=L$ and $z_{1}=\gamma(t)$. Then $\operatorname{dist}_{\mathrm{qh}}\left(z_{1}, z\right)=L-g(t)$. By the inequality $(31)$,

$$
\frac{d}{d t} \operatorname{length}\left(\gamma\left(z_{1}, z\right)\right)=-1 \geq-\frac{d}{d t} g(t) H(g(t))=\frac{d}{d t} \int_{\operatorname{dist}_{\mathrm{qh}}\left(z, z_{0}\right)}^{\infty} H(r) d r .
$$

Integrating from $t$ to $L$, we obtain that

$$
-\operatorname{length}\left(\gamma\left(z_{1}, z\right)\right) \geq-\int_{\operatorname{dist}_{\mathrm{qh}}\left(z, z_{0}\right)}^{\infty} H(r) d r+\int_{L}^{\infty} H(r) d r
$$

and

$$
\text { length }\left(\gamma\left(z_{1}, z\right)\right) \leq \int_{\operatorname{dist}_{\mathrm{qh}}\left(z, z_{0}\right)}^{\infty} H(r) d r
$$

Set $A(r)$ to be the inverse of $\int_{r}^{\infty} H(r) d r$. Then $A(r)$ is a non-decreasing continuous function and $\lim _{r \rightarrow 0} A(r)=\infty$. The lemma follows.

Corollary 11.1 For every integrable domain $\Omega$ there exists a continuous function $A$ defined in Lemma 11.4 so that for every subarc $\gamma_{1}$ of a minimal quasihyperbolic geodesic $\gamma$ starting at the base point $z_{0}$ the following inequality holds

$$
\frac{\operatorname{length}\left(\gamma_{1}\right)}{A\left(\text { length }\left(\gamma_{1}\right)\right)} \lesssim \max _{z \in \gamma_{1}} \operatorname{dist}(z, \partial \Omega) \text {. }
$$




\subsection{Local connectivity and continua of convergence}

A connected set $K$ is locally connected if for every $z \in K$ and each neighborhood $U$ of $z$ there exists a neighborhood $V$ of $z$ such that $K \cap V$ lies in a single component of $K \cap U$.

Definition 11.1 A continuum $K_{\infty} \subset M$ is called a continuum of convergence of a set $M$ if there exists a sequence of continua $K_{i} \subset M$ so that

1. $K_{i}$ are pairwise disjoint for $i=1, \ldots, \infty$.

2. $\lim _{i \rightarrow \infty} K_{i}=K_{\infty}$ in the Haudorff metric.

A concept of continuum of convergence has a principal application in study of local connectedness of continua. We will say that a continuum of convergence is non-trivial if it contains more than one point. We have the following fact (see Theorems 12.1 and 12.3 in [49]) which follows almost immediately from the definition of local connectivity.

Fact 11.1 If a continuum $M$ is not locally connected then there exists a non-trivial continuum of convergence of $M$.

From Fact 11.1, it is clear that the local continuity of $M$ cannot fail just in one point. It fails for all points from a non-trivial continuum of convergence.

Lemma 11.5 The boundary of an integrable domain $\Omega$ (which is not necessarily connected) does not have a non-trivial continuum of convergence.

Proof: Suppose that there exists a non-trivial continuum of convergence $K_{\infty} \subset \partial \Omega$. Let $K_{i} \rightarrow K_{\infty}$ be mutually disjoint continua of $\partial \Omega$. We choose a point $w$ in $K_{\infty}$ and $\epsilon>0$ so that the circle $\{|z-w|=\epsilon\}$ intersects $K_{\infty}$ in at least two points and $\Omega \backslash \overline{B_{\epsilon}(w)}$ is non-empty. Without loss of generality, every $K_{i}$ intersects $\{|z-w|=\epsilon\}$ in at least two points and hence $B_{\epsilon}(w) \backslash K_{i}$ has at least two components. For every $n>1 / \epsilon$ choose a component $\Omega_{n}$ of $\Omega \cap B_{\epsilon}(w)$ and $z_{n} \in \Omega_{n}$ with the following properties: (i) dist $\left(z_{n}, w\right)=1 / n$, (ii) there exists $j$ such that $w$ and $z_{n}$ are in different components of $B_{\epsilon}(w) \backslash K_{j}$. Observe that there are infinitely many different $\Omega_{n}$. Indeed, let $\Gamma_{j}$ be a component of $B_{\epsilon}(w) \backslash K_{j}$ which contains $w$. By (ii), there exists $j$ so that $\Omega_{n}$ is contained in a component of $B_{\epsilon}(w) \backslash K_{j}$ different than $\Gamma_{j}$. By (i), there exists $N>n$ so that $z_{N}$ and thus $\Omega_{N}$ are contained in $\Gamma_{j}$. Consequently, every $\Omega_{m}$ with $m>N$ and $\Omega_{n}$ are different.

By the connectivity of $\Omega, \partial \Omega_{n} \cap\{|z-w|=\epsilon\} \neq \emptyset$. We join every $z_{n}$ with a base point $z_{0} \in \Omega \backslash \overline{B_{\epsilon}(w)}$ by a minimal quasihyperbolic geodesic $\gamma\left(z_{n}, z_{0}\right)$. Let $\gamma_{n} \ni z_{n}$ be a component of $\gamma\left(z_{n}, z_{0}\right) \cap B_{\epsilon / 2}(w)$. The Euclidean length of $\gamma_{n}$ is at least $\epsilon / 4$ for large $n$. Then, by Corollary 11.1,

$$
\max _{y \in \gamma_{n}} \operatorname{dist}\left(y, \partial \Omega_{n}\right) \geq \max _{y \in \gamma_{n}} \operatorname{dist}(y, \partial \Omega) \gtrsim \frac{\operatorname{length}\left(\gamma_{i}\right)}{A\left(\operatorname{length}\left(\gamma_{i}\right)\right)} \gtrsim \frac{\epsilon}{4 A(\epsilon / 4)}=: \delta .
$$

Consequently, every $\Omega_{n}$ contains a ball of radius $\delta$ and hence there are infinitely many disjoint balls of the same radius in $B_{\epsilon}(w)$, a contradiction. 
The non-existence of a non-trivial continuum of convergence implies immediately that every component of the boundary is locally connected. We see immediately that every subcontinuum of a continuum without non-trivial continuum of convergence is locally connected. In fact it gives even more precise topological description of every component of $\partial \mathcal{F}$ (see [49] pp 82-84).

Definition 11.2 We say that a continuum $K$ is a rational curve if each point $z \in K$ has a family of arbitrary small neighborhoods $U_{n}$ so that $\partial U_{n} \cap K$ is countable.

Theorem 3.3 and Remark 2.1 of Chapter V of [49] assert that every continuum which has no nontrivial continuum of convergence is a rational curve. Another direct consequence of Lemma 11.5 is that for every $\epsilon>0$ there exists only finitely many components $\partial \mathcal{F}$ with diameters larger than $\epsilon$. This in turn will imply the absence of wandering continua for polynomials which satisfy the summability condition.

\subsection{Wandering continua}

Definition 11.3 A continuum $K$ is called wandering if for every two non-negative integers $m \neq n$

$$
F^{m}(K) \cap F^{n}(K)=\emptyset \text {. }
$$

Lemma 11.6 Suppose that $F$ satisfies the summability condition with exponent $\alpha<\frac{1}{1+\mu_{\max }}$ and $\mathcal{F}$ is a periodic Fatou component. Then $F$ has no wandering continua contained in $\partial \mathcal{F}$ other than points. In particular, if $F$ is a polynomial then every non-point component of connectivity of $J$ is preperiodic.

Proof: We can always assume that $K$ is connected. Let $R^{\prime}$ be supplied by Proposition 7.2. We look at the orbit $K_{i}:=F^{i}(K)$. Since $\partial \mathcal{F}$ does not have a non-trivial continuum of convergence almost all continua $K_{i}$ have the diameter smaller than $R^{\prime} / 2$. A ball $B_{i}$ of the radius $R^{\prime}$ centered at any point of $K_{i}$ contains $K_{i}$. By Proposition 7.2, we obtain that

$$
\operatorname{diam} K \leq \operatorname{diam} F^{-i}\left(B_{i}\right)<\left(\tilde{\omega_{n}}\right)^{-1} \rightarrow 0
$$

and $K$ must be a point.

\section{Uniform summability condition}

Continuity of scales and parameters. Suppose that $F$ is a rational function which satisfies the summability condition with an exponent $\alpha$ and a sequence of rational functions $F_{i}$ tends $S(\alpha)$-uniformly to $F$. By the definition of $S(\alpha)$-uniform convergence and Lemma 2.2, we choose the same sequences $\left\{\alpha_{n}\right\},\left\{\gamma_{n}\right\}$, and $\left\{\delta_{n}\right\}$ for all $F_{i}$ and $F$. 
All constants defined in (i) - (iii) in Section 2.3 can be chosen uniformly that is independently from $F_{i}$ for $i$ large enough. The condition (iv) from Section 2.3 needs to be replaced by the condition $\left(\right.$ iv $\left.^{\prime}\right)$ :

$\left(\right.$ iv $\left.^{\prime}\right)$ Let Crit $_{F}^{\prime}$ be a set of critical points of $F$ outside the Julia set $J_{F}$. We chose $R^{\prime}$ is so small that for all $i$ large enough there are no critical points Crit $_{F}^{\prime}$ in the $2 R^{\prime}$-neighborhood of the Julia set $J_{i}$.

This choice of $R^{\prime}$ is indeed possible. Since $F$ and $F_{i}, i \geq 0$, satisfy the summability condition all periodic orbits of $F$ and $F_{i}, i \geq 0$, are either repelling or attracting, see Corollary 2.2. Suppose now that we can find a sequence $c_{i_{k}}$ of critical points of $F_{i_{k}}$ such that for some $c \in \mathrm{Crit}_{F}^{\prime}$

$$
\operatorname{dist}\left(c_{i_{k}}, c\right) \rightarrow 0 \text { and } \operatorname{dist}\left(c_{i_{k}}, J_{i_{k}}\right) \rightarrow 0
$$

when $k \rightarrow \infty$. The critical point $c$ is in the basin of an attracting periodic point $w$. Let $\eta>0$. There is $m>0$ such that dist $\left(F^{m}(c), w\right)<\eta / 3$. For every $i$ large enough there is an attracting periodic point $w_{i}$ of $F_{i}$ such that dist $\left(w_{i}, w\right)<\eta / 3$. Since $\operatorname{dist}\left(F^{m}\left(c_{i_{k}}\right), J_{i_{k}}\right) \rightarrow 0$ when $k \rightarrow \infty$, we have that for all $k$ large enough $\operatorname{dist}\left(J_{i_{k}}, w_{i_{k}}\right)<\eta$, a contradiction.

Uniform version of Lemma 2.4. We will argue that after the above choice of the sequences, the constants and scales (i) - (iv'), all the constants $R^{\prime}, R_{2 t}, L, C(q), K$ from Lemma 2.4 are uniform, that is independent from $F_{i}$ for $i$ large enough.

1. We say that a sequence $F^{-n}(z), \ldots F^{-1}(z), z$, of type 2 preimages of $z$ is in the scale $R^{\prime}$ if the ball $B_{R^{\prime} / 2}(z)$ can be pulled back univalently along the orbit $F^{-n}(z), \cdots, F^{-1}(z), z$ and $\operatorname{dist}\left(z, J_{F}\right) \leq R^{\prime} / 2$.

We claim that for every $L_{u}^{\prime}>0$ there exists a neighborhood of $F$ in the space of rational functions with the topology of uniform convergence so that for every $G$ from that neighborhood and every sequence $G^{-L_{u}^{\prime}}(z), \cdots, z$ of type 2 preimages of $z$ in the scale $R^{\prime}$ and such that $\operatorname{dist}\left(z, J_{G}\right)<R^{\prime} / 16$, the corresponding sequence $F^{-L_{u}^{\prime}}(z), \cdots, z$ is of type 2 in the scale $R^{\prime} / 2$. Indeed, if $G$ close enough to $F$ then $J_{F}$ is contained in $R^{\prime} / 8$-neighborhood of $J_{G}$. To see this consider all repelling periodic orbits of $F$ with periods smaller than a constant and the property that they are $R^{\prime} / 16$-dense in $J_{F}$. By the implicit function theorem $J_{G}$ is $R^{\prime} / 4$ dense in $J_{F}$ if $G$ is close to $F$.

We possibly increase the constant $L$ from Lemma 2.4 so that the first two claims of Lemma 2.4 are satisfied with the estimates 7 and $1 / 37$ instead of 6 and $1 / 36$, respectively, for all type 2 preimages of $z$ by $F$ in the scale $R^{\prime} / 2$. We put $L_{u}:=L$.

Therefore, by $C^{1}$ continuity for every $L_{u}^{\prime}>L_{u}$ there exists a neighborhood of $F$ so that for every $G$ from this neighborhood and every sequence of type 2 preimages of $z$ in the scale $R^{\prime}$,

$$
\inf _{y \in \mathbf{I I}(z), L_{u}^{\prime} \geq n(y) \geq L_{u}}\left|\left(G^{n}\right)^{\prime}(y)\right|>6
$$


and if the Poincaré series $\Sigma_{q}(v)$ for $F$ converges for some point $v$ then

$$
\sum_{y \in \mathbf{I I}(z), L_{u}^{\prime} \geq n(y) \geq L_{u}}\left|\left(G^{n}\right)^{\prime}(y)\right|^{-q}<\frac{1}{36}
$$

provided $\operatorname{dist}\left(z, J_{G}\right)<R^{\prime} / 16$.

2. The constants $K, R_{2 t}$, and $C(q)$ of Lemma 2.4 come from the Koebe type estimates and depend only on other uniform constants hence are uniform.

We formulate a uniform version of Lemma 3.1.

Lemma 12.1 Let $F$ be a rational function which satisfies the summability condition with an exponent $\alpha \leq 1$ and $\left(F_{i}\right)$ be a sequence of rational maps converging $S(\alpha)$-uniformly to $F$. There exists $\epsilon>0$ such that the backward orbit $\cdots, F_{i}^{-k}(z), \cdots, z$ of any $z$ from the $\epsilon$-neighborhood of the Julia set $J_{i}$ stays in the $R^{\prime} / 16$-neighborhood of $J_{i}$. The same claim is true for $F$.

Proof: The existence of $\epsilon_{i}>0$ for every $F_{i}$ is proved in Lemma 3.1. If $\epsilon_{i}$ tend to 0 then $J_{F} \neq \hat{\mathbb{C}}$. Suppose that there is an infinite set $I$ of positive integers such that for every $i \in I$ there are $z_{i} \in \hat{\mathbb{C}}$ and $n_{i} \in \mathbb{N}$ so that dist $\left(z_{i}, J_{i}\right) \geq R^{\prime} / 16$ and $\operatorname{dist}\left(F_{i}^{n_{i}}\left(z_{i}\right), J_{i}\right) \leq \epsilon_{i}$. By the compactness, there exists a converging sequence $z_{i_{j}} \rightarrow z \in \hat{\mathbb{C}}$ with the property that $B_{R^{\prime} / 32}(z)$ is disjoint with every Julia set $J_{i_{j}}, i_{j} \in I$, and thus also disjoint from $J_{F}$. This means that there is $m>0$ such that $F^{m}(z)$ is in the immediate bassin of attraction of an attracting periodic point $w$ of $F$. Let $w_{i_{j}}$ be the corresponding attracting periodic point of $F_{i_{j}}$ for $j$ large enough. By the continuity, $F_{i_{j}}^{m}(z)$ are uniformly close to $w_{i_{j}}$ and therefore the orbit of $z$ by $F_{i_{j}}$ must accumulate on the periodic orbit of $w_{i_{j}}$ for all $j$ large enough, a contradiction.

Lemma 12.2 (Uniform version of the Main Lemma) Assume that a rational function $F$ satisfies the summability condition with an exponent $\alpha \leq 1$ and set $\beta=\mu_{\max } \alpha /(1-\alpha)$. Suppose that $F_{i}$ is a sequence of rational functions tending $S(\alpha)$-uniformly to $F$. Let $\epsilon$ be supplied by Lemma 12.1 and suppose that a point $z$ belongs to the $\epsilon$-neighborhood of the Julia set $J_{i}$ and a ball $B_{\Delta}(z)$ can be pulled back univalently by a branch of $F_{i}^{-N}$.

We claim that there exists $i_{0}$ and positive constants $L^{\prime}>L, K$ independent of $z, \Delta$, and $\epsilon$ such that for every $i \geq i_{0}$ the sequence $F_{i}^{-N}(z), \ldots, z$ can be decomposed into blocks of types 1 , 2 , and 3 , and

- every type 2 block, except possibly the leftmost one, has the length contained in $\left[L, L^{\prime}\right)$ and yields expansion 6 ,

- the leftmost type 2 block has the length contained in $[0, L]$ and yields expansion $K>0$,

- all subsequences of the form 1...13, except possibly the rightmost one, yield expansion

$$
\gamma_{k_{j}} \ldots \gamma_{k_{1}} \gamma_{k_{0}}
$$

$k_{i}$ being the lengths of the corresponding blocks, 
- the rightmost sequence of the form $1 \ldots 13$ yields expansion

$$
\begin{array}{ll}
\gamma_{k_{j}} \ldots \gamma_{k_{1}} \gamma_{k_{0}} \Delta^{\left(1-\mu(c) / \mu_{\max }\right)} & \text { if a critical point } c \in B_{\Delta}(z) \\
\gamma_{k_{j}} \ldots \gamma_{k_{1}} \gamma_{k_{0}} \Delta^{\left(1-1 / \mu_{\max }\right)} & \text { if otherwise. }
\end{array}
$$

Proof: We claim that the inductive construction from the Main Lemma can be carried out for $F_{i}$ and any $z$ from the $\epsilon$-neighborhood of $J_{i}$ provided $i$ is large enough. The constants and scales are given by the conditions (i) - (iv'). Recall that the same sequences $\left\{\alpha_{n}\right\},\left\{\gamma_{n}\right\}$, and $\left\{\delta_{n}\right\}$ can be used for both $F_{i}$ and $F$. By the definition of $S(\alpha)$-uniform convergence, there is a $1-1$ correspondence between the critical points of $F$ and these of $F_{i}$ if only $i$ is large enough. We follow the inductive decomposition of backward orbits of $F_{i}$ of the Main Lemma with one exception, all the critical points of $F_{i}$ contained in the $\epsilon$-neighborhood of $J_{i}$ are included in the construction. We treat $F_{i}$ as small perturbations of $F$ and work in the same scale $0<R^{\prime}<R$ both for $F_{i}$ and $F$. Using the uniform summability condition for all critical points inside the $\epsilon$-neighborhood of $J_{i}$, we obtain the expansion from the last three claims of Lemma 12.2 in exactly the same way as in the proof of the Main Lemma. In the construction of all backward pieces of type $1 \ldots 13$ we use all the critical points of $F_{i}$ from the $\epsilon$-neighborhood of $J_{i}$.

What remains is the proof that we can find $L$ and $L^{\prime}>L$ independently from $F_{i}$ so that the first two claims of Lemma 12.2 hold. We put $L:=L_{u}$ and hence $L$ is independent from $F_{i}$ by the uniform version of Lemma 2.4. Next, for a given $F_{i}$, the constant $L^{\prime}$ in the proof of the Main Lemma is defined as $L+L^{\prime \prime}$ where $L^{\prime \prime}$ is a function of $\left\{\alpha_{n}\right\}, C_{3 t}$, and $R_{2 t}$ only. These are the uniform constants. We conclude the proof by invoking the uniform version of Lemma 2.4 for $L_{u}$ and $L_{u}^{\prime}=L_{u}+L^{\prime \prime}$.

Uniform self-improving property and main estimates. The proof of Proposition 4.1 is self-contained except for references to Lemmas 3.4 and 3.5. Suppose that rational functions $F_{i}$ converge $S(\alpha)$-uniformly to a rational function $F$ which satifies the summability condition with an exponent $\alpha \leq 1$. By the uniform version of the Main Lemma and Lemma 12.1, we obtain the estimates of Lemma 3.4 for all $F_{i}$ sufficiently close to $F$. The decomposition of backward orbits of $F_{i}$ into pieces of type 1,2, and 3 uses, as in the uniform version of the Main Lemma, all the critical points of $F_{i}$ contained in the $\epsilon$-neighborhood of $J_{i}$ ( $\epsilon$ comes from Lemma 12.1). Lemma 3.5 is a reformulation of two claims of Lemma 2.4 which already has a uniform version. The constants $L:=L_{u}^{\prime}$ and $L^{\prime}:=L_{u}^{\prime}$ are supplied by the uniform version of the Main Lemma.

Now suppose as in the hypothesis of Proposition 4.1 that $F$ satisfies the summability condition with an exponent $\alpha<q /\left(\mu_{\max }+q\right)$ for some $q>0$ and that the sequence $\left(F_{i}\right)$ converges $S(\alpha)$-uniformly to $F$. Also, assume that the Poincaré series for $F$ with an exponent $q>0$ converges for some point $v \in \hat{\mathbb{C}}, \Sigma_{q}(v)<\infty$. In the proof of Proposition 4.1 the assumption $\Sigma_{q}(v)<\infty$ is used to derive the following property: there exists $\eta>0$ so that for every $z$ such that $\operatorname{dist}\left(z, J_{F}\right)<\eta$,

$$
\sum_{y \in \mathbf{I I}_{l}(z)}\left|\left(F^{n(y)}\right)^{\prime}(y)\right|^{-q}<\frac{1}{36},
$$


where $\mathbf{I I}_{l}(z)$ is the set of all "long" (of order $L^{\prime}>n(y) \geq L$ ) type 2 preimages $y$ of $z$. The property in fact holds for all $F_{i}$ close enough to $F$ by the uniform version of Lemma 2.4 with $\eta:=\epsilon$ of Lemma 12.1. This shows that the self-improving property of Proposition 4.1 is true for all $F_{i}$ close enough to $F$.

Taking into account the uniform self-improving property and Corollary 4.1, we obtain the main estimate behind the continuity of the Hausdorff dimension of Julia sets for $S(\alpha)$-uniformly converging rational maps.

Corollary 12.1 Assume that $F$ satisfies the summability condition with an exponent

$$
\alpha<\frac{p}{\mu_{\max }+p}
$$

$p$, and a sequence of rational maps $\left(F_{i}\right)$ converges $S(\alpha)$-uniformly to $F$. If there exist $\epsilon>0$ and $q>p$ so that for every point $z$ from the $\epsilon$-neighborhood of the Julia set $J_{F}$,

$$
\sum_{y \in \mathbf{I I}_{l}(z)}\left|\left(F^{n(y)}\right)^{\prime}(y)\right|^{-q}<\frac{1}{36},
$$

then for every $i$ large enough $\delta_{\text {Poin }}\left(J_{i}\right)<q$.

Corollary 12.2 If a sequence $\left(F_{i}\right)$ of rational functions tends $S(\alpha)$-uniformly to $F$ which satisfies the summability condition with an exponent $\alpha<\frac{\delta_{\text {Poin }}\left(J_{F}\right)}{\mu_{\max }+\delta_{P o i n}\left(J_{F}\right)}$ then

$$
\limsup _{i \rightarrow \infty} \delta_{\text {Poin }}\left(J_{i}\right) \leq \delta_{\text {Poin }}\left(J_{F}\right)
$$

Proof: By Theorem 1, for every positive $\eta$ and every critical point of maximal multiplicity, the Poincaré series for $F, \Sigma_{\delta_{\text {Poin }}\left(J_{F}\right)+\eta}(c)<\infty$. By the uniform version of Lemma 2.4, for every $\eta>0$ we can find $L_{u}(\eta) \leq L_{u}^{\prime}(\eta)$ so that for every $z$ such that $\operatorname{dist}\left(z, J_{i}\right)<\epsilon(\epsilon$ is supplied by Lemma 12.1),

$$
\sum_{y \in \mathbf{I I}(z), L_{u}(\eta)^{\prime} \geq n(y) \geq L_{u}(\eta)}\left|\left(F_{i}^{n}\right)^{\prime}(y)\right|^{-\delta_{\text {Poin }}\left(J_{F}\right)-\eta}<\frac{1}{36}
$$

provided $i$ is large enough. $\mathbf{I I}(z)$ stands for a set of type 2 preimages of $z$ for $F_{i}$. In the definition of these type 2 preimages all critical points of $F_{i}$ inside the $\epsilon$-neighborhood of $J_{i}$ were considered. The constants $L_{u}^{\prime}(\eta)>L_{u}(\eta)$ depend only on $F$ and $\eta$. We use the uniform version of Corollary 12.1 (set $p:=\delta_{\text {Poin }}(J)$ and $\left.q:=\delta_{\text {Poin }}(J)+\eta\right)$ to conclude that $\delta_{\text {Poin }}\left(J_{i}\right) \leq$ $\delta_{\text {Poin }}(J)+\eta$ for all $i$ large enough. 
Proof of Theorem 12. By Corollary 12.2 and Theorem 1,

$$
\limsup _{n \rightarrow \infty} \operatorname{HDim}\left(J_{n}\right)=\limsup _{n \rightarrow \infty} \delta_{\text {Poin }}\left(J_{n}\right) \leq \delta_{\text {Poin }}\left(J_{F}\right)=\operatorname{HDim}\left(J_{F}\right) .
$$

Since $\operatorname{HDim}\left(J_{F}\right)=\operatorname{HypDim}\left(J_{F}\right)$, for every $\eta>0$ there exists a hyperbolic subset $K$ of $J_{F}$ so that $\operatorname{HDim}(K) \geq \operatorname{HDim}\left(J_{F}\right)-\eta$ (see $\left.[12,13]\right)$. By the general theory of hyperbolic sets, the set $K$ persists under small perturbations and therefore every $J_{i}$ for $i$ large enough contains a hyperbolic set $K_{i}$ of the Hausdorff dimension $\operatorname{HDim}(K)-2 \eta$. Consequently,

$$
\lim \inf _{i \rightarrow \infty} \operatorname{HDim}\left(J_{i}\right) \geq \operatorname{HDim}\left(J_{F}\right),
$$

which proves the theorem.

\section{Unicritical polynomials}

It is known that the connectedness locus $\mathcal{M}_{d}$ for unicritical polynomials $z^{d}+c$ is a full compact. Let $\phi$ be the Riemann map from the unit disk to $\mathbb{C} \backslash \mathcal{M}_{d}$. By Fatou's theorem for almost all $\xi$ with respect to the Lebesgue measure on the unit circle, there exists a radial $\operatorname{limit} \lim _{r \rightarrow 1} \phi(r \xi)$. Therefore, the harmonic measure $\chi$ is given by,

$$
\chi=\phi_{*}(m) .
$$

By Fatou's theorem, for almost every $c \in \partial \mathcal{M}_{d}$ with respect to $\chi$ the external radius $\Gamma(c)$ (see Definition 1.9) terminates at $c$.

Denote the Julia set of the polynomial $z^{d}+c$ by $J_{c}$. By Shishikura's theorem, [41], there exists a residual set $Z \subset \partial \mathcal{M}_{2}$ with the property that $\forall c \in Z$,

$$
\operatorname{HypDim}\left(J_{c}\right)=2 \text {. }
$$

Let $c \in \partial \mathcal{M}_{2}$ corresponds to a Collet-Eckmann polynomial. By [20] and [42], Collet-Eckmann parameters are typical with respect to the harmonic measure $\chi$ and the corresponding Julia sets are of Hausdorff dimension $<2$, [18]. Choose now a sequence $c_{n} \in Z, c_{n} \rightarrow c$. Since HypDim(.) is lower semicontinuous, there exist open disks $D_{n}$ centered at $c_{n}$ so that $\forall c \in D_{n}$,

$$
\operatorname{HDim}\left(J_{c_{n}}\right)>2-\frac{1}{n} \text {. }
$$

By Yoccoz's theorem, $\mathcal{M}_{2}$ is locally connected at $c$ and thus there exists a curve $\gamma$ terminating at $c$ so that

$$
\limsup _{\gamma \ni c^{\prime} \rightarrow c} \operatorname{HDim}\left(J_{c^{\prime}}\right)=2>\operatorname{HDim}\left(J_{c}\right)
$$

and HDim as a function of $c^{\prime} \in \mathbb{C} \backslash \mathcal{M}_{2}$ does not extend continuously to $\partial \mathcal{M}_{2}$.

Another type of discontinuity of $\operatorname{HDim}(\cdot)$ is caused by the parabolic implosion. Let $c \in \partial \mathcal{M}_{2}$, $c \in \Gamma(c)$, has a parabolic cycle. The parabolic implosion means that $\operatorname{HDim}\left(J_{c}\right)$ is strictly contained in the Hausdorff limit of $J_{c^{\prime}}, c^{\prime} \in \Gamma(c)$. It was recently shown in [14] that if $d=2$ and $c>1 / 4$ then

$$
\operatorname{HDim}\left(J_{1 / 4}\right)<\liminf _{c \rightarrow 1 / 4} \operatorname{HDim}\left(J_{c}\right) \leq \limsup _{c \rightarrow 1 / 4} \operatorname{HDim}\left(J_{c}\right)<2 .
$$




\subsection{Renormalizations}

We will start with the observation unicritical polynomials satisfying the summability condition are not infinitely renormalizable.

Lemma 13.1 Suppose that $f_{c}$ satisfies the summability condition with an exponent $\alpha \leq \frac{1}{1+d}$. Then $f_{c}$ is only finitely many times renormalizable.

Proof: Indeed, suppose that $f:=f_{c}$ is infinitely renormalizable. Then there is a sequence $n_{j}$ and two topological disks $\overline{U_{j}} \subset V_{j}$ so that $f^{n_{j}}: U_{j} \rightarrow V_{j}$ is proper of degree $\ell$ and

$$
J_{j}:=\cap_{i=0}^{\infty} f^{-n_{j} i}\left(U_{j}\right) \ni 0
$$

is a non-trivial continuum $\left(f^{-n_{j}}\right.$ is a branch which sends $V_{j}$ onto $\left.U_{j}\right)$. We may assume that $\forall j \geq 0, U_{j+1} \subset U_{j}$. Let $R^{\prime}$ be a constant chosen in Section 2.3. If for every $j$ positive and every $0 \leq k \leq n_{j}$, diam $f^{k}\left(J_{j}\right) \geq R^{\prime}$ then $\cap_{j}^{\infty} J_{j}$ is a non-trivial wandering continuum and the Julia set of $f_{c}$ would not be locally connected. Hence, there exist $j$ and $k$ so that $\operatorname{diam} f^{k}\left(J_{j}\right) \leq R^{\prime}$. Let $B_{R^{\prime}} \supset f^{k}\left(J_{j}\right)$. We apply Proposition 7.2 for the inverse branches $f^{-k-n_{j} s}, s$ is a positive integer, $f^{-n_{j} s}\left(B_{R^{\prime}}\right) \supset J_{j}$, and the ball $B_{R^{\prime}}$. Then

$$
\operatorname{diam} J_{j}=\operatorname{diam} f^{-k-n_{j} s}\left(B_{R^{\prime}}\right) \leq \omega_{k+n_{j} s}^{-1} \rightarrow 0,
$$

a contradiction.

\subsection{Radial limits}

Proof of Theorem 13. Let $f_{c}=z^{d}+c$. We use the following fact stated as Theorem 1.2 in $[20]$.

Fact 13.1 Let $\Gamma\left(c_{0}\right)$ be an external ray landing at $c_{0}$. For every $d \geq 2$ and for almost every $c_{0} \in \partial \mathcal{M}_{d}$ with respect to the harmonic measure there exist constants $K>0$ and $\Lambda>1$ so that for each $c \in \Gamma\left(c_{0}\right)$ and every $n>0$

$$
\left(f_{c}^{n}\right)^{\prime}(c) \geq K \Lambda^{n}
$$

This means that for almost all $c_{0}$ with respect to the harmonic measure, $f_{c}, c \in \Gamma\left(c_{0}\right)$, converges $S(\alpha)$-uniformly to $f_{c_{0}}$ for every $\alpha>0$. To complete the proof of Theorem 13, we invoke Theorem 12,

$$
\limsup _{\Gamma\left(c_{0}\right) \ni c \rightarrow c_{0}} \operatorname{HDim}\left(J_{c}\right)=\operatorname{HDim}\left(J_{c_{0}}\right) .
$$


Proof of Theorem 14. Fix a generic $c_{0} \in \partial \mathcal{M}_{d}$ as in the proof of Theorem 13. Consider $f_{c}$ with $c \in \Gamma\left(c_{0}\right)$. Since $f_{c}$ is hyperbolic, by [45] it admits a unique conformal measure $\nu_{c}$ which is a Hausdorff measure (restricted to the Julia set $J_{c}$ ) normalized by a multiplicative constant so that its total mass is one. Hence, the conformal exponent of $\nu_{c}$ is equal to $\operatorname{HDim}\left(J_{c}\right)$. By Theorem 13, $\operatorname{HDim}\left(J_{c}\right) \rightarrow \operatorname{HDim}\left(J_{c_{0}}\right)$ as $\Gamma\left(c_{0}\right) \ni c \rightarrow c_{0}$. This means that any weak accumulation point of $\nu_{c}$ is a conformal measure with an exponent $\operatorname{HDim}\left(J_{c_{0}}\right)$. But there is only one such a measure for $f_{c_{0}}$ by Theorem 2, and we obtain the convergence of $\nu_{c}$ to the geometric measure of $f_{c_{0}}$. 


\section{References}

[1] M. Aspenberg The Collet-Eckmann condition for rational maps on the Riemann Sphere. Ph. D. Thesis, KTH Sweden, 2004.

[2] A. F. Beardon. Iteration of rational functions. Springer-Verlag, New York, 1991.

[3] M. Benedicks, L. Carleson. On iterations of $1-a x^{2}$ on $(-1,1)$. Ann. of Math. (2), 122(1):1-25, 1985.

[4] M. Benedicks, L. Carleson. The dynamics of the Hénon map. Ann. of Math. (2), 133(1):73$169,1991$.

[5] C. J. Bishop. Minkowski dimension and the Poincaré exponent. Michigan Math. J., 43(2):231-246, 1996.

[6] C. J. Bishop and P. W. Jones. Hausdorff dimension and Kleinian groups. Acta Math., 179(1):1-39, 1997.

[7] H. Bruin, S. van Strien Expansion of derivatives in one-dimensional dynamics. Israel J. Math. 137 (2003), 223-263.

[8] H. Bruin, S. Luzzatto, S. van Strien Decay of correlations in one-dimensional dynamics. Ann. Sci. cole Norm. Sup. (4) 36 (2003), no. 4, 621-646.

[9] H. Bruin, W. Shen, S. van Strien Invariant measures exist without a growth condition. Comm. Math. Phys. 241 (2003), no. 2-3, 287-306.

[10] L. Carleson, T. W. Gamelin. Complex dynamics. Springer-Verlag, New York, 1993.

[11] W. de Melo and S. van Strien. One-dimensional dynamics. Springer-Verlag, Berlin, 1993.

[12] M. Denker, M. Urbański. On Sullivan's conformal measures for rational maps of the Riemann sphere. Nonlinearity, 4(2):365-384, 1991.

[13] M. Denker, M. Urbański. On the existence of conformal measures. Trans. Amer. Math. Soc., 328(2):563-587, 1991.

[14] A. Douady, P. Sentenac, M. Zinsmeister. Implosion parabolique et dimension de Hausdorff. C. R. Acad. Sci. Paris Sér. I Math., 325(7):765-772, 1997.

[15] H. Federer. Geometric measure theory. Springer-Verlag New York Inc., New York, 1969. Die Grundlehren der mathematischen Wissenschaften, Band 153.

[16] F. W. Gehring, O. Martio. Lipschitz classes and quasiconformal mappings. Ann. Acad. Sci. Fenn. Ser. A I Math., 10:203-219, 1985. 
[17] J. Graczyk, D. Sands, G. Świątek. Metric Attractors for Smooth Unimodal Maps. Ann. Math. (2), 159(2):725-740, 2004.

[18] J. Graczyk, S. Smirnov. Collet, Eckmann and Hölder. Invent. Math., 133(1):69-96, 1998.

[19] J. Graczyk, G. Świątek. The real Fatou conjecture. Princeton University Press, Princeton, NJ, 1998.

[20] J. Graczyk, G. Świątek. Harmonic measure and expansion on the boundary of the connectedness locus. Invent. Math., 142(3):605-629, 2000.

[21] G. H. Hardy, J. E. Littlewood, G. Pólya. Inequalities. Cambridge University Press, Cambridge, 1988. Reprint of the 1952 edition.

[22] J. Heinonen, P. Koskela. Definitions of quasiconformality. Invent. Math., 120(1):61-79, 1995.

[23] M. V. Jakobson. Absolutely continuous invariant measures for one-parameter families of one-dimensional maps. Comm. Math. Phys., 81(1):39-88, 1981.

[24] P. W. Jones, S. K. Smirnov. Removability theorems for Sobolev functions and quasiconformal maps. Ark. Mat., 38(2): 263-279, 2000.

[25] S. Kallunki, P. Koskela. Exceptional sets for the definition of quasiconformality. Amer. J. Math., 122(4):735-743, 2000.

[26] O. Kozlovski, W. Shen, S. van Strien. Rigidity for real polynomials. Ann. of Math., (2) 165 (2007), no. 3, 749-841.

[27] P. Koskela. Old and new on the quasihyperbolic metric. In Quasiconformal mappings and analysis (Ann Arbor, MI, 1995), pages 205-219. Springer, New York, 1998.

[28] G. Levin, S. van Strien. Local connectivity of the Julia set of real polynomials. Ann. of Math. (2), 147(3):471-541, 1998.

[29] R. Mañé, P. Sad, D. Sullivan. On the dynamics of rational maps. Ann. Sci. École Norm. Sup. (4), 16(2):193-217, 1983.

[30] R. Mañé. The Hausdorff dimension of invariant probabilities of rational maps. In Dynamical systems (Valparaiso, 1986), pages 86-117. Springer, Berlin-New York, 1988. Lecture Notes in Mathematics, Vol. 1331.

[31] P. Mattila. Geometry of sets and measures in Euclidian spaces. Cambridge University Press, Cambridge, 1995.

[32] C. T. McMullen. Complex dynamics and renormalization. Princeton University Press, Princeton, NJ, 1994. 
[33] C. T. McMullen. Hausdorff dimension and conformal dynamics. II. Geometrically finite rational maps. Comment. Math. Helv. 75(4): 535-593, 2000.

[34] T. Nowicki, S. van Strien. Invariant measures exist under a summability condition for unimodal maps. Invent. Math., 105(1):123-136, 1991.

[35] Ch. Pommerenke. Boundary behavior of conformal maps. Springer-Verlag, New York, 1992.

[36] F. Przytycki. On measure and Hausdorff dimension of Julia sets of holomorphic ColletEckmann maps. In International Conference on Dynamical Systems (Montevideo, 1995), pages 167-181. Longman, Harlow, 1996.

[37] F. Przytycki. Iterations of holomorphic Collet-Eckmann maps: conformal and invariant measures. Appendix: on non-renormalizable quadratic polynomials. Trans. Amer. Math. Soc., 350(2):717-742, 1998.

[38] F. Przytycki. Conical limit set and Poincaré exponent for iterations of rational functions. Trans. Amer. Math. Soc., 351(5): 2081-2099, 1999.

[39] F. Przytycki, S. Rohde. Rigidity of holomorphic Collet-Eckmann repellers. Ark. Mat., 37(2):357-371, 1999.

[40] M. Rees. Positive measure sets of ergodic rational maps. Ann. Sci. École Norm. Sup. (4), 19(3):383-407, 1986.

[41] M. Shishikura. The Hausdorff dimension of the boundary of the Mandelbrot set and Julia sets. Ann. of Math. (2), 147(2):225-267, 1998.

[42] S. Smirnov. Symbolic dynamics and Collet-Eckmann conditions. Internat. Math. Res. Notices, 2000(7):333-351.

[43] W. Smith and D. A. Stegenga. Hölder domains and Poincaré domains. Trans. Amer. Math. Soc., 319(1):67-100, 1990.

[44] E. M. Stein. Singular integrals and differentiability properties of functions. Princeton University Press, Princeton, N.J., 1970. Princeton Mathematical Series, No. 30.

[45] D. Sullivan. Conformal dynamical systems. In Geometric dynamics (Rio de Janeiro, 1981), pages 725-752. Springer, Berlin, 1983.

[46] M. Tsujii. Positive Lyapunov exponents in families of one-dimensional dynamical systems. Invent. Math., 111(1):113-137, 1993.

[47] M. Urbański. Measures and dimensions in conformal dynamics. Bull. Amer. Math. Soc., 40: 281-321, 2003. 
[48] P. Walters. Ergodic theory - introductory lectures. Springer-Verlag, Berlin-New York, 1975. Lecture Notes in Mathematics, Vol. 458.

[49] G. T. Whyburn. Analytic topology. American Mathematical Society, Providence, R.I., 1963. 